\title{
WIND FARM POWER PREDICTION IN COMPLEX TERRAIN
}

\author{
by \\ Micah Sandusky
}

\author{
A thesis \\ submitted in partial fulfillment \\ of the requirements for the degree of \\ Master of Science in Mechanical Engineering \\ Boise State University
}

August 2017 
(C) 2017

Micah Sandusky

ALL RIGHTS RESERVED 


\title{
DEFENSE COMMITTEE AND FINAL READING APPROVALS
}

\author{
of the thesis submitted by
}

\author{
Micah Sandusky
}

Thesis Title: Wind Farm Power Prediction in Complex Terrain

Date of Final Oral Examination: 19 June 2017

The following individuals read and discussed the thesis submitted by student Micah Sandusky, and they evaluated his presentation and response to questions during the final oral examination. They found that the student passed the final oral examination.

Inanc Senocak, Ph.D.

Ralph S. Budwig, Ph.D.

Trevor Lujan, Ph.D.

Clare Fitzpatrick, Ph.D.
Chair, Supervisory Committee

Member, Supervisory Committee

Member, Supervisory Committee

Member, Supervisory Committee

The final reading approval of the thesis was granted by Inanc Senocak, Ph.D., Chair of the Supervisory Committee. The thesis was approved by the Graduate College. 
dedicated to my parents and Annie. 


\section{ACKNOWLEDGMENTS}

There are many people I would like to thank for helping and supporting me throughout my studies. Dr. Inanc Senocak gave me the opportunity to work in research four and a half years ago and has helped to shape my academic success ever since. He has spent innumerable hours teaching me, editing my papers, and troubleshooting my work. He deserves my largest thanks. I would like to thank my committee members Dr. Ralph Budwig, Dr. Trevor Lujan, and Dr. Clare Fitzpatrick for taking the time and effort to be on my committee and for the valuable feedback.

I would also like to thank everyone in the High Performance Simulation Lab for Thermo-Fluids (HiPerSimLab). Specifically, Rey DeLeon was a great mentor from the beginning and helped me throughout my time in research at Boise State. Jason Cook deserves my thanks for his excellent work as systems administrator of the Kestrel HPC cluster.

The Idaho Space Grant Consortium (ISGC) deserves my thanks for awarding me a NASA EPSCoR fellowship that funded my research in part. The research presented in this thesis is also based upon work supported by the National Science Foundation under Grant No. 1056110 and 1229709. 


\begin{abstract}
There has been increasing interest in predicting the velocity field within wind farms in complex terrain for resource assessment, turbine siting, and power forecasting. These capabilities are made possible by advancements in computational speed from a new generation of computing hardware and numerical methods. The current thesis research focuses on two technical components to advance the current state in wind power forecasting. The first component is improved prediction of wind flow over complex terrain using the versatile immersed boundary method to represent surface boundary conditions on a fixed Cartesian mesh. The proposed approach embodies the law-of-the-wall for rough surfaces and produces good results for benchmark wind data for complex terrain. The second component is the implementation and validation of wind turbine wake models and a first-principle based method to predict available wind power. Actuator disk models with and without rotation are considered. The wake models are validated against data from a published wind tunnel experiment and full-scale field data from an operational wind farm. The power prediction method is compared against normalized power data from operational wind farms and other computational studies available in literature. The actuator disk model with rotation simulates wake velocity deficits with better accuracy than the non-rotational model. The proposed power prediction method shows agreement with standard energy assessment methods without any ad-hoc decisions. Finally, the computational capability is applied to a hypothetical wind farm in Southern Idaho to demonstrate its versatility.
\end{abstract}




\section{TABLE OF CONTENTS}

ABSTRACT $\ldots \ldots \ldots \ldots \ldots \ldots \ldots \ldots \ldots \ldots \ldots \ldots \ldots \ldots \ldots \ldots \ldots$

LIST OF FIGURES $\ldots \ldots \ldots \ldots \ldots \ldots \ldots \ldots \ldots$ ix

LIST OF TABLES $\ldots \ldots \ldots \ldots \ldots \ldots \ldots \ldots \ldots \ldots \ldots \ldots \ldots \ldots \ldots \ldots \ldots$

1 Introduction $\ldots \ldots \ldots \ldots \ldots \ldots \ldots \ldots \ldots \ldots \ldots \ldots \ldots \ldots \ldots \ldots \ldots$

1.1 Wind Prediction Models and Their Applications ............... 4

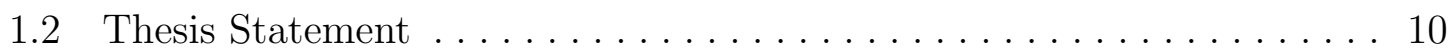

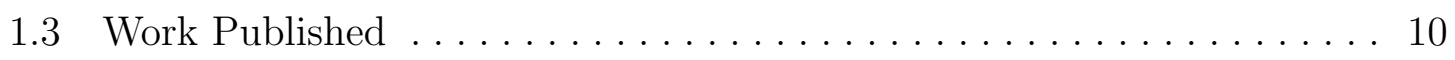

2 Wind prediction over complex terrain $\ldots \ldots \ldots \ldots \ldots \ldots \ldots \ldots$

2.1 Wind Resource Assessment $\ldots \ldots \ldots \ldots \ldots \ldots \ldots \ldots \ldots \ldots$

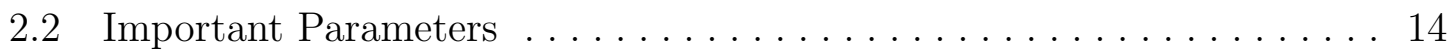

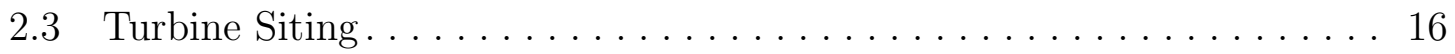

2.4 Wind Farm Design $\ldots \ldots \ldots \ldots \ldots \ldots \ldots \ldots \ldots \ldots \ldots \ldots \ldots \ldots \ldots \ldots$

2.5 Wind Power Forecasting .......................... 18

2.6 Current State of Wind Simulations $\ldots \ldots \ldots \ldots \ldots \ldots \ldots \ldots \ldots$

3 Technical Background $\ldots \ldots \ldots \ldots \ldots \ldots \ldots \ldots \ldots \ldots \ldots \ldots \ldots \ldots \ldots \ldots$

3.1 Immersed Boundary Method $\ldots \ldots \ldots \ldots \ldots \ldots \ldots \ldots \ldots \ldots \ldots$

3.2 Governing Equations $\ldots \ldots \ldots \ldots \ldots \ldots \ldots \ldots \ldots \ldots \ldots \ldots \ldots \ldots \ldots \ldots$

3.3 Numerical Methods $\ldots \ldots \ldots \ldots \ldots \ldots \ldots \ldots \ldots \ldots \ldots \ldots$ 
4 Wind Farm Modeling $\ldots \ldots \ldots \ldots \ldots \ldots \ldots \ldots \ldots \ldots \ldots \ldots \ldots \ldots \ldots \ldots \ldots$

4.1 Wind Turbine Wake Models . . . . . . . . . . . . . . . . . . . 29

4.1.1 Actuator Disk Model with No Rotation . . . . . . . . . . 29

4.1.2 Actuator Disk Model with Rotation . . . . . . . . . . . . 31

4.1 .3 Actuator Line Model. . . . . . . . . . . . . . . . . . . . 34

4.2 Computer Implementation $\ldots \ldots \ldots \ldots \ldots \ldots \ldots \ldots \ldots \ldots \ldots$

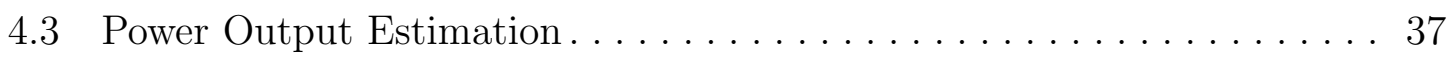

$5 \quad \operatorname{Results\ldots \ldots \ldots \ldots \ldots \ldots \ldots \ldots \ldots \ldots \ldots \ldots \ldots \ldots }$

5.1 Wind Flow over Complex Terrain $\ldots \ldots \ldots \ldots \ldots \ldots \ldots \ldots$

5.1 .1 Bolund Hill Full Scale . . . . . . . . . . . . . . . . . . 44

5.1 .2 Bolund Hill Wind Tunnel $\ldots \ldots \ldots \ldots \ldots \ldots \ldots \ldots \ldots$

5.1 .3 Askervein Hill . . . . . . . . . . . . . . . . . . . 47

$5.2 \quad$ Wake Models ............................... 50

5.3 Control Volume Analysis for Power Estimation ................ 53

5.4 Application to a Wind Farm over Complex Terrain .............. 62

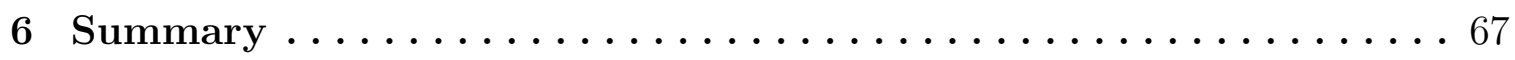

6.1 Wind Over Complex Terrain $\ldots \ldots \ldots \ldots \ldots \ldots \ldots \ldots \ldots \ldots$

6.2 Wake Models . .......................... 67

6.3 Control Volume Analysis for Power Estimation .............. 68

6.4 Future Work .................................. 69

REFERENCES......................... 70 


\section{LIST OF FIGURES}

1.1 Increase in wind turbine size 1980-2011. Image is adopted from [29] under the Creative Commons license and is public domain. . . . . . . . 2

1.2 Schematic of the structure of the ABL. Image is adopted from [45] under the Creative Commons license. Image is based on [60]. . . . . . . 3

1.3 A sketch of the incoming wind profiles in complex terrain subject to different thermal stability conditions. . . . . . . . . . . . . . . .

1.4 Normalized turbine power for each row of Horns Rev wind farm when the flow direction is aligned with turbine rows. Data is from [5]. The $\mathrm{X}$-axis displays the turbine row and the $\mathrm{Y}$-axis measures power normalized by power at the first row . . . . . . . . . . . 6

2.1 NREL wind map showing annual average wind speeds at 80 meters above ground level across the United States Figure is adopted from [30]. This map was created by the National Renewable Energy Laboratory for the U.S. Department of Energy with data provided by AWS TruePower.12

2.2 Observed difference between mast extrapolation and SODAR measurements. Image is adopted from AWS TruePower, a UL Company. [8] . . . 14

$2.340 \mathrm{~m}$ isosurface of velocity over Perdigao hill in Portugal. . . . . . . . . . 16

2.4 Example of variation in fractional speedup, $\Delta S$, along line A from the Bolund Blind Study results. Profiles are given at $2 \mathrm{~m}$ and $5 \mathrm{~m}$ above the hill. Plot is adopted from Bechmann et al. [6]. . . . . . . . . . . . 20 
3.1 IB reconstruction in the surface normal direction. $\alpha, \beta, \gamma$, and $\delta$ are the Cartesian nodes used to interpolate onto FI. TC is the triangle centroid, $I B$ is the immersed boundary node, and FI is the cell face intersection. Interpolation occurs along the projected line from $T C$, through $I B$, to $F I . \ldots \ldots \ldots \ldots \ldots \ldots \ldots \ldots \ldots \ldots \ldots \ldots \ldots$

3.2 Visualization of the distance field from the complex terrain of Hells Canyon, Idaho. . . . . . . . . . . . . . . . . . . . . . 25

3.3 Schematic of the energy spectra for turbulent flows. . . . . . . . 26

4.1 Plot of power as a function of wind speed for the Suzlon S88-2.1 MW wind turbine. Data is from $[62] \ldots \ldots \ldots \ldots \ldots \ldots \ldots \ldots \ldots \ldots$

4.2 Visualization of the stream tube boundary around the actuator disk showing the fractional relationship between incoming velocity and the axial induction factor. Image is adapted from $[34] \ldots \ldots \ldots \ldots \ldots 31$

4.3 Plot showing the relationship between $a$ and the thrust and power coefficients. Image is adapted from $[34] \ldots \ldots \ldots \ldots \ldots \ldots \ldots \ldots$

4.4 Visualization of an airfoil cross-section showing the relative forces, angles, and velocities for the BEM approach. $\gamma$ is the local pitch angle, $\alpha$ is the angle of attack, and $\phi$ is the angle of relative wind at which $U_{r e l}$ approaches. Image is adapted from [50]. . . . . . . . . . . 34

4.5 Visualization the blade element mesh overlaid on the Cartesian mesh. $V_{x}$ and $V_{\theta}$ are the axial and rotational components of the velocity, the uses of which are detailed in [34]. $r$ is the radius at each blade element. Image is adapted from $[72] . \ldots \ldots \ldots \ldots \ldots \ldots \ldots \ldots \ldots \ldots \ldots$

5.1 Elevation map of Bolund Hill with mast locations and wind direction. . 45 
5.2 Fractional speedup for full scale mesh study along line B with $270^{\circ}$ wind direction compared with full scale field data [6] vertical profiles at masts $7,6,3$, and 8 . Mesh 1 is the most coarse. . . . . . . . . 46

5.3 Fractional speedup of scale simulation along line B with $270^{\circ}$ wind direction compared with wind tunnel [76], water tunnel, and full scale [6] vertical profiles at masts $7,6,3$, and $8 \ldots \ldots \ldots \ldots \ldots$

5.4 Visualization of velocity field around Askervein Hill showing the measurement profiles. . . . . . . . . . . . . . . . . . . . . . . 49

5.5 Fractional speed up along Askervein AA Line showing sensitivity to RANS-LES blending height, $h_{B}$. The field data is from [69]. . . . . . 50

5.6 Fractional speed up along Askervein A Line showing sensitivity to RANS-LES blending height, $h_{B}$. The field data is from [69]. . . . . . 51

5.7 Fractional speed up along Askervein AA Line showing sensitivity to mesh resolutions in Table 5.2. The field data is from [69] . . . . . . . . 52

5.8 Fractional speed up along Askervein A Line showing sensitivity to mesh resolution in Table 5.2 . The field data is from $[69] \ldots \ldots \ldots \ldots$. . . . . 53

5.9 Streamwise velocity profiles wind turbine wind tunnel experiment and simulations. $Z / D$ refers to height above ground level normalized by the turbine diameter. Results are shown from the wind tunnel experiment from Chamorro and Porté-Agel [12], ADM-NR and ADM-R simulations [71], and the ADM-NR and ADM-R results from the current study using GIN3D. The velocity profiles are at 3D (3 diameters), 5D, and 10D downstream as well as 1D upstream. . . . . . . . . . . 54 
5.10 Wind turbine and SODAR station locations for the subset of the wind farm in Mower County. S1 and S2 represent SODAR stations and T39-T43 represent turbines. Image is adopted from [50]. . . . . . . . . . 55

$5.11 C_{L}$ curve as a function of radius as reported from the $3 \mathrm{D}$ simulations in Laursen et al. [31]. Data is from [31] . . . . . . . . . . . . . . . . 56

5.12 Streamwise velocity profile results showing simulation and field data results for the wind farm in Mower County. Results are shown for the SODAR stations, the ADM-NR, ADM-R, and ALM simulations from Porté-Agel [50], and the ADM-NR and ADM-R simulations performed with GIN3D. . . . . . . . . . . . . . . . . . . . . . . . 57

5.13 Streamwise velocity profiles at SODAR station S1 in the Mower County. Results are shown for the SODAR stations, Porté-Agel et al. simulation [50], the log-law profile, and the current study simulations performed with GIN3D using the turbulent perturbation inflow method. 58

$5.14 C_{L}$ and $C_{D}$ curves as a function angle of attack as reported in [72].

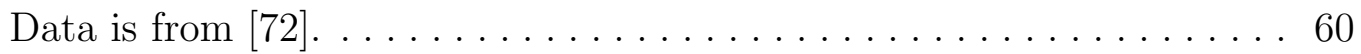

5.15 Incoming velocity profile for the Horns Rev wind farm showing the current study and the velocity profile in $\mathrm{Wu}$ et al. [72] . . . . . . . . . 61

5.16 Visualization of the Horns Rev wind farm mean flow field showing the wind farm dimensions. . . . . . . . . . . . . . . . . . . 62

5.17 Visualization of the Horns Rev wind farm instantaneous flow field showing the wind farm dimensions. . . . . . . . . . . . . 63

5.18 Visualization of the mean flow field around the first column of turbines in the Horns Rev wind farm. 
5.19 Visualization of the instantaneous flow field around the first column of turbines in the Horns Rev wind farm. . . . . . . . . . . . . . . . 64

5.20 Reported angular speed as a function of wind speed for the Vestas V80 turbine. Data is from $[72] \ldots \ldots \ldots \ldots \ldots \ldots \ldots \ldots$

5.21 Average power at each turbine row normalized by power at row 1 . Field data is reported in Barthelmie et al. [4] and the previous ADM-R simulation and WindSim [70] industry software results are reported in

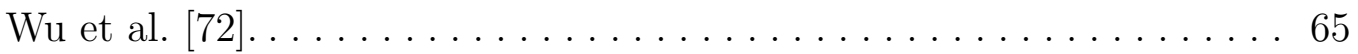

5.22 Visulization of the turbines, complex terrain, and flow field for a hypothetical wind farm in Hagerman, Idaho. . . . . . . . . . . . . . . 66 


\section{LIST OF TABLES}

2.1 IEC 61400 standards for turbine classes. Table is adapted from [10] . . . 15

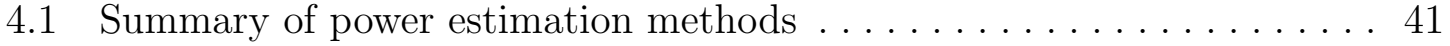

5.1 Summary of simulation parameters for Field Study (FS) and Wind Tunnel $(\mathrm{WT})$ cases. . . . . . . . . . . . . . . . . . . . . . 44

5.2 Summary of mesh sizes for Field Study (FS) and Wind Tunnel (WT) cases. . . . . . . . . . . . . . . . . . . . . . . . . . 44

5.3 Summary of percent power drop from T41 to T42 in the Mower County wind farm simulation $\ldots \ldots \ldots \ldots \ldots \ldots \ldots$

5.4 Summary of power losses from test Case 1 (4.3D turbine spacing) and Case 2 (7.3D turbine spacing). . . . . . . . . . . . . . . . . 59

5.5 Results from the investigation of variation in power drop with control volume size. . . . . . . . . . . . . . . . . . . . . . . . . . . . . . 59 


\section{CHAPTER 1}

\section{INTRODUCTION}

Wind power has made strides in the last few decades. The amount of development in wind power suggests that it has become economically profitable [18]. In fact, the U.S. has over 52,000 operational utility-scale wind turbines and an installed capacity of $82,183 \mathrm{MW}$ [3] and Idaho produces $15.2 \%$ of its total energy from wind power [2].

In order to increase power production, wind turbines have grown in size. The power extracted from a turbine is directly proportional to the square of the rotor radius and the cube of the velocity at hub height, so increase in rotor size and height creates exponential power gains. The increase in turbine size in recent years is shown in Figure 1.1. At this point, the swept area of new wind turbines has surpassed the wingspan of many large aircrafts. The Enercon E126 7.5 MW turbine has a rotor diameter of $127 \mathrm{~m}$, which is larger than the wingspan of a Boeing 747 .

The size of the current wind turbines means that most of the flow they are exposed to is contained in the lowest part of the atmospheric boundary layer (ABL), referred to as the surface layer, which extends as high as 100 to $200 \mathrm{~m}$ from the ground. ABL structure can be seen in Figure 1.2.

ABL is the lower part of Earth's atmosphere. It extends to about $1000 \mathrm{~m}$ from ground level and exists under the free atmosphere. Virtually every part of our daily life exists within the ABL, and most of our life exists within the surface layer. Its structure 


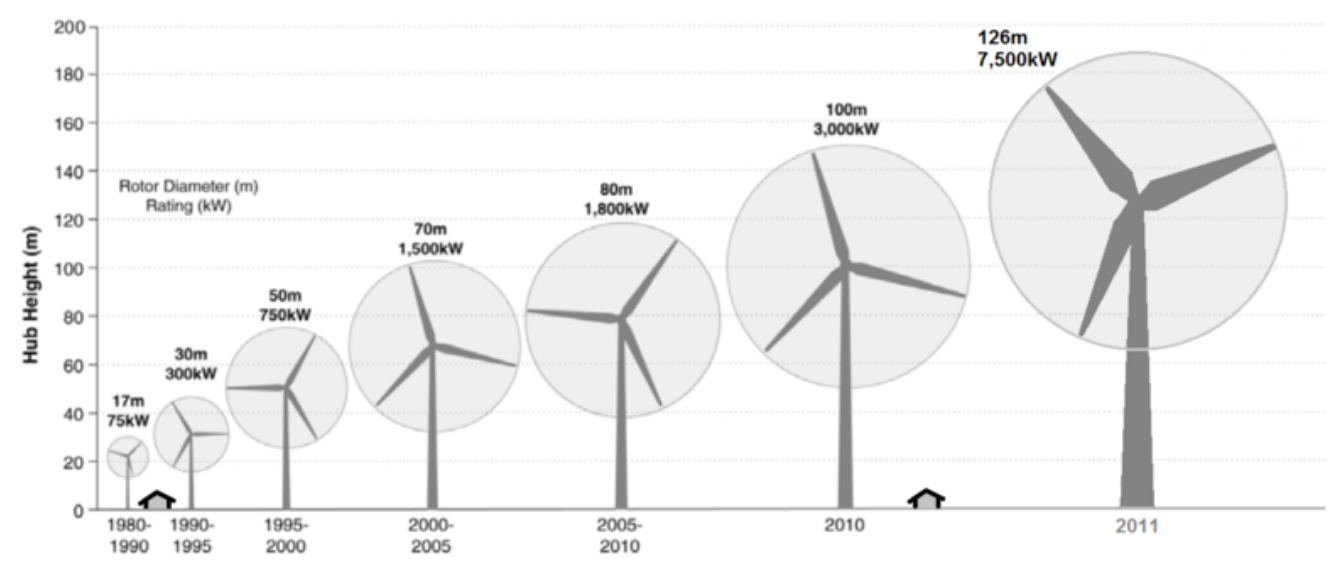

Figure 1.1: Increase in wind turbine size 1980-2011. Image is adopted from [29] under the Creative Commons license and is public domain.

is unlike the aerodynamic boundary layer due to the large scale and is dominated by interactions with the Earth. ABL can be categorized by three structures: unstable stratification, stable stratification, and neutral stratification. The velocity profile for the surface layer is

$$
U=\frac{u^{*}}{\kappa} \ln \frac{h}{z_{0}}+\psi\left(z, z_{0}, L\right)
$$

In this equation, $U$ is the velocity, $u^{*}$ is the friction velocity, $\kappa$ is the von Kármán constant equal to $0.41, h$ is the elevation above ground, $z_{0}$ is the aerodynamic surface roughness, and $\psi\left(z, z_{0}, L\right)$ is the stability term.

As the sun causes surface heating throughout the day, the air closest the ground becomes hotter and less dense. As the less dense air rises, the reduced pressure causes expansion and adiabatic cooling. If thermal equilibrium with the surrounding air cannot be achieved by the cooling, the air will keep rising, which can cause convective cells [10]. This is classified as unstable stratification. When the ground cools at night, the rising air can end up cooler than the surrounding air due to the adiabatic cooling 


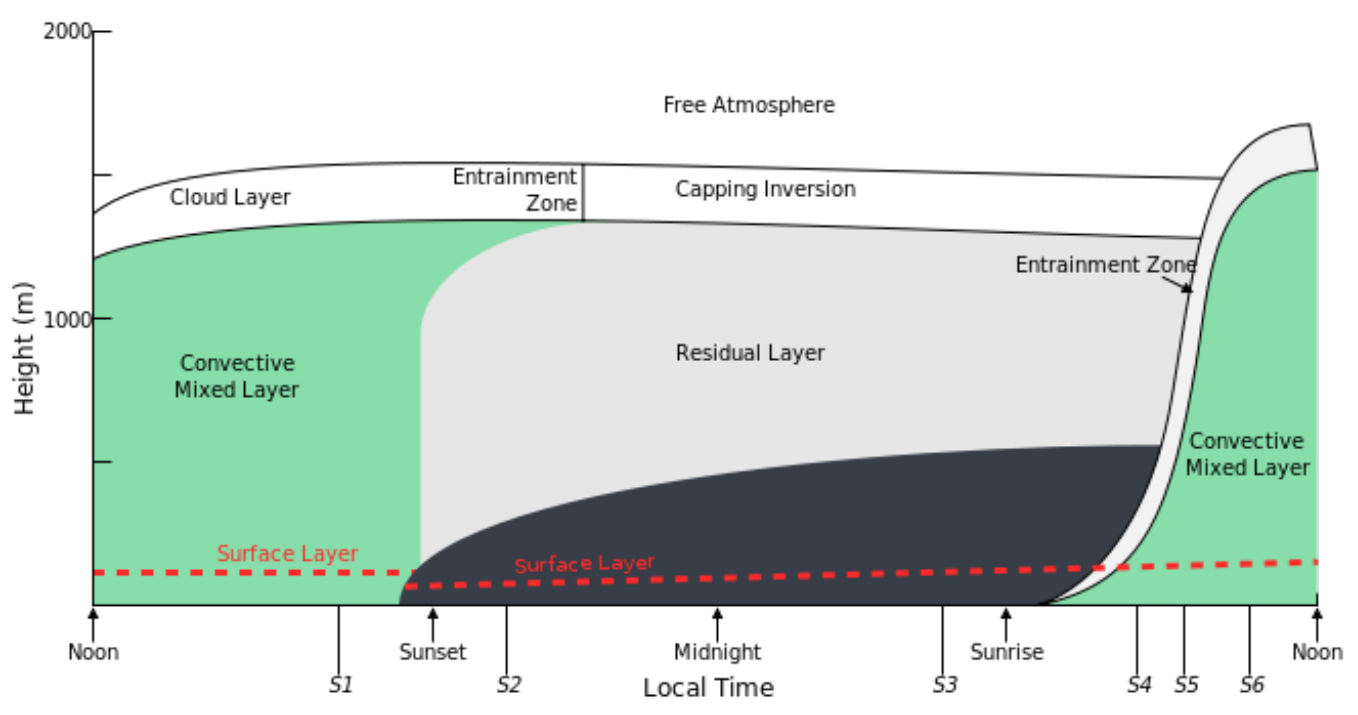

Figure 1.2: Schematic of the structure of the ABL. Image is adopted from [45] under the Creative Commons license. Image is based on [60].

[10]. This hinders the rising of the air, causing what is known as stable stratification. This structure often forms a low level jet phenomenon as depicted in Figure 1.3. When the rising air maintains thermal equilibrium with the surrounding air as it rises, the structure is known as neutral stratification [10]. The stability term can be neglected for the neutral condition. The neutral structure is the most commonly assumed situation for wind simulations to avoid complications of a stratified atmosphere. It also often accompanies strong winds [10].

Wind turbine aerodynamics are similar to lifting-body (e.g. wing) aerodynamics, but with further complications due to the size and placement within the surface layer of the atmospheric boundary layer. Turbines are exposed to severe shear profiles (change in stream wise velocity with increasing height) from the ground, complex wake interactions, and very unsteady winds, which are rarely experienced by airplane wings. Due to the complexities of complex terrain, most expansion in wind power has been on flat terrain and offshore. These locations have a much more predictable 


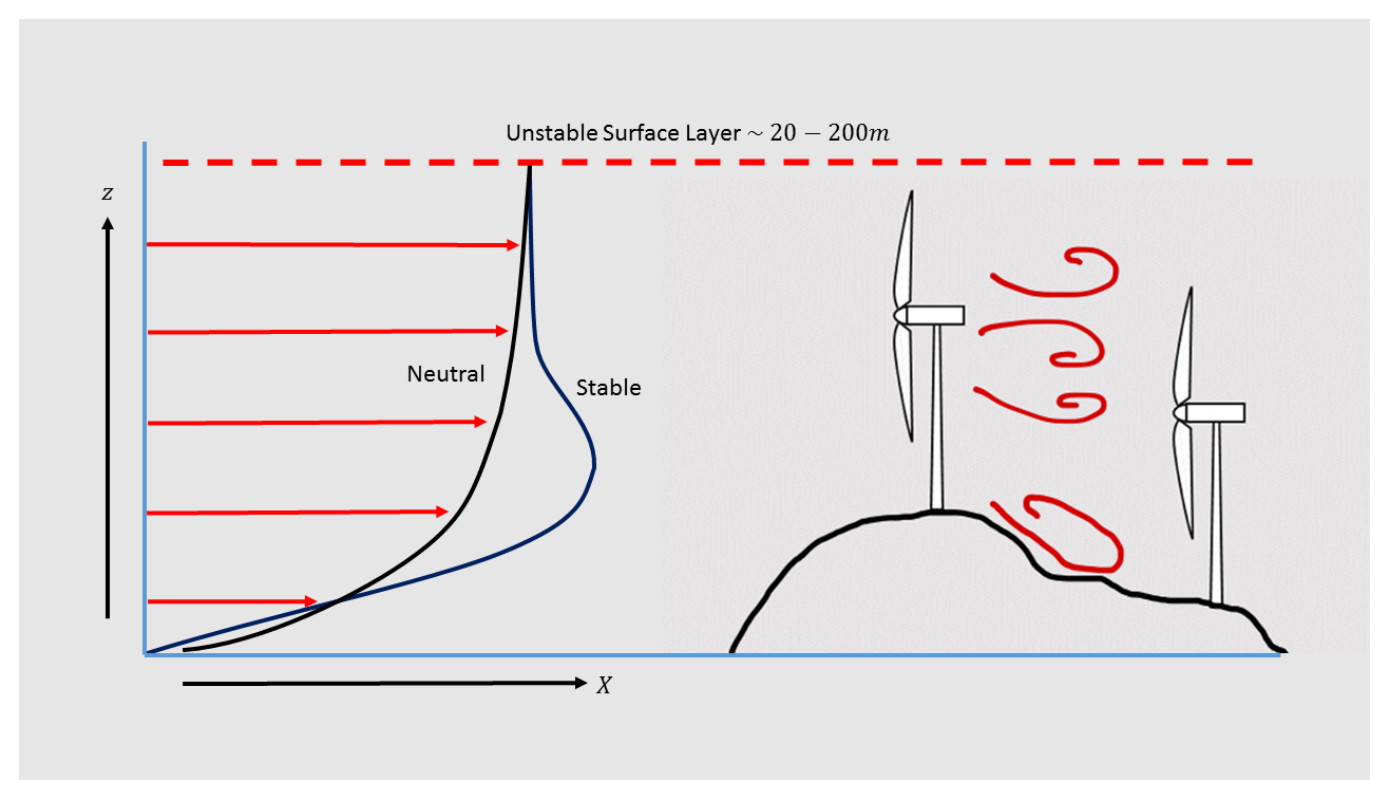

Figure 1.3: A sketch of the incoming wind profiles in complex terrain subject to different thermal stability conditions.

ABL than mountainous and complex terrain, and a more predictable power output. Since much of the practical flat terrain is already developed and installing new transmission lines is both difficult and expensive, there has been increased interest around installing wind farms in complex terrain. Complex terrain flow interactions are not well understood, but features such as hilltops, ridges, and mesas can provide desirable flow for wind turbines [9]. Furthermore, the western United States is primarily composed of complex terrain, and could be a promising area for more wind power expansion. After all, the world is not flat.

\subsection{Wind Prediction Models and Their Applications}

When it comes to wind farms, power prediction is an important task. Wake effects play a large role in wind farm performance. Investigations into wind farm wake effects show that there is a disproportionate drop in power after the first turbine and smaller 
percentage power loss after subsequent turbines, especially when the turbine rows are aligned parallel to the wind flow $[5,59]$. This is shown in Figure 1.4. This is caused by an increase vertical flux of kinetic energy due to increased wake turbulence [59].

Incoming turbulence can have drastic effects on turbine wake behavior, which is very important to consider in wind farms $[4,11,59]$. Large scale turbulent fluctuations have measurable effects on power, whereas smaller scale fluctuations have an effect on the strain at the base of the tower [11]. Wind tunnel experiments of near wake regions show that tip vortices are present for 2-3 diameters past the turbine and the wake grows laterally behind the turbine [77]. There is a lack of deep understanding of wake interactions in large wind farms, and this already complex system will become even more difficult when moved into a complex terrain environment. Another issue with harvesting wind power is farm to farm interactions. Wind farms remove a significant amount of momentum from the air. If one developer decides to build a farm upwind from another farm, this could cause serious viability issues for the already existing farm. It is near impossible to understand the real impact of a new development, or even wake interactions within existing farms, without a comprehensive simulation capability to evaluate both the airflow and power output.

Power output within wind farms is inherently unsteady due to changes in wind speed and direction throughout the days, months, and even years. Additionally, the power grid must be balanced to constantly meet the power demand. As a result, fast-responding generators must be kept on standby to fill in any gaps that result from unforeseen wind shortages. This is inefficient and causes wind power to not fulfill its capabilities. A more accurate prediction of wind farms would result in less reliance on non-renewable energy sources for balancing the generation and load on the grid and would increase the value of wind energy. Currently, some combination 


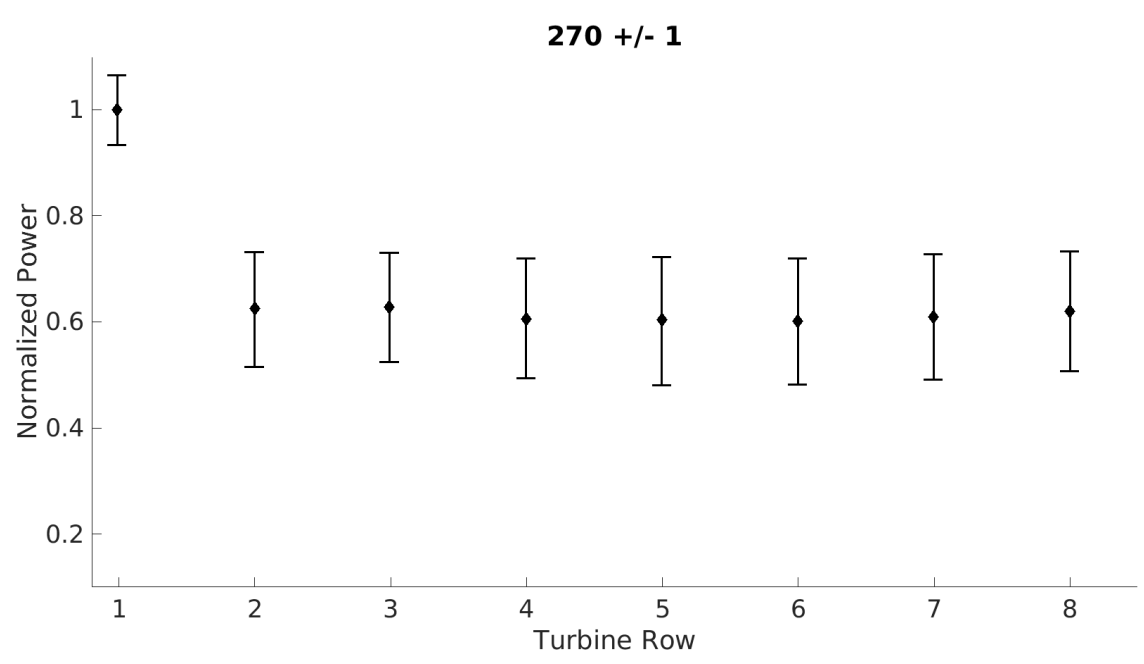

Figure 1.4: Normalized turbine power for each row of Horns Rev wind farm when the flow direction is aligned with turbine rows. Data is from [5]. The $\mathrm{X}$-axis displays the turbine row and the $\mathrm{Y}$-axis measures power normalized by power at the first row.

of statistical tools and Numerical weather prediction models (NWPs) are used to aid in wind power forecasting. Statistical methods are generally used for very short term forecasts, whereas climatology models are better for forecasting wind speed at more than 15 hours [23]. Cheng et al. showed some improvement to NWP models for wind speed prediction by assimilating anemometer data [13]. A couple issues with these methods are that a small error in velocity can show large errors in wind power due to the nonlinearity of the turbine power curves and resource assessment becomes difficult due to the variation in environmental conditions [23]. Additionally, they do not consider the effects of turbine wakes.

The combination of innovative numerical methods and increasing computational power has opened the door to high detail, large area simulations of atmospheric flow over complex train. The immersed boundary (IB) method enables efficient flow calculations over large terrain areas and the resulting computational data fits efficiently to the memory architecture of graphics processing units (GPU). Computationally, it 
is possible to achieve real-time predictions of flow over complex terrain.

Physics-based turbine wake models have been developed for computational fluid dynamics (CFD) applications that have been shown to accurately predict wind power. The most common wake models have been the actuator disk model with no rotation and with rotation (ADM-NR and ADM-R) and the actuator line model (ALM) [50, 71]. The ADM-R and the ALM are based off of Blade Element Momentum theory, which utilizes a momentum balance around the rotor as well as airfoil theory to properly characterize thrust and power [34].

There has been notable exploration into the application of these wake models in literature in recent years. The ADM-NR consistently shows good far wake agreement but poor near wake agreement and a failure to capture the overall wake structure in simulations of both wind tunnel and full scale experiments [50, 71]. The ADM-R and the ALM both capture the far and near wake velocity drop [36, 50, 58, 71] and both have consistent success with power predictions, within $1 \%$ of each other [36]. Induced rotation is necessary for accurate wake behavior and power prediction from turbine models. The ALM does a better job than ADM-NR and ADM-R of capturing the power, wake, turbulent kinetic energy (TKE) and tip vortices $[36,50,58]$. Both rotational models show promise for accurate simulation of wind turbines without resolving the turbine blades.

Studies have been performed to use these models to better understand wake effects in wind farms and in complex terrain. One study compared use of an large eddy simulation (LES) and ADM-R model to the Horns Rev wind farm and found overall good agreement with the large sector cases [72]. It was found that the incoming wind direction had a large effect on velocity and turbulence intensity within the farm. Using similar methods, it was discovered that increasing stream-wise turbine spacing has 
more ability to increase power than increasing span-wise spacing [74]. Beyond effects within the wind farms, there is a notable effect on the global and local meteorology. Simulating wind farms using the effective roughness technique becomes impractical for larger turbines and can induce large errors as it is not suited to simulate the surface layer [33]. The ALM has been used to simulate large ideal wind farms and investigate wake effects, finding a discernible effect on the global local meteorology from such a farm. One study applied the ADM-R model with a modeled relationship between shaft torque and rotational speed to the Horns Rev offshore wind farm in Denmark [72]. They found good power agreement with the proposed model whereas there was and under-prediction in power for the industry software WindSim [70] and WAsP [21]. This study shows significant promise for use of LES simulations and wake models as a predictive and design tool, but little work has been done to expand these methods to complex terrain and wind power prediction.

Complex terrain can have a large effect on wind turbine wake behavior. One study looked at the effects on wake behavior of an artificial upstream hill at 3 different heights. When the hill was taller than hub height, the turbine experienced faster wake recovery, more even distribution of TKE in the far wake region, and an overall increase in TKE [73]. Another study that compared wind farm wakes in complex terrain and on flat terrain showed some of the significant differences [75]. The study used the Virtual Wind Simulator (VWiS) software framework and the ALM, as well as the IB method, but did not perform any validation on the complex terrain simulations. Another study investigated wind power in complex terrain using the ADM-NR model and two different softwares [48]. They found correlation between terrain and wake recovery, most likely due to increased turbulence intensity. They also found significant differences in the turbine wakes depending on which software was used. Rodrigo 
et al. summarized the important verification methods for flow-over-terrain model comparisons with regard to wind power [52].

Realistic inflow conditions are needed to accurately predict wind turbine wakes and power. Interesting phenomenon occur due to the unsteadiness of turbine wakes and wind farms, similar to the disproportionate drop in power after the first turbine discussed previously. The unsteadiness of inflow turbulence has large effects on power output, making power prediction and grid balancing difficult, which is one of the reasons that improved simulation of wind farms in complex terrain is one of the future issues for the study of wind power [59]. Additionally, wind farm power output can show correlations with neighboring farms over hundreds of kilometers [59]. As Barthelmie et al. observes, the thrust and power curve are necessary for modeling of wakes, but vary with the particular environment [4]. Also, there is debate on whether the thrust coefficient should be set to a uniform for an entire wind farm, or be unique at each turbine [4]. There is a need for more exploration into the application of wind power models to create a better understanding of wake behavior and to advance wind resource assessment methods.

There are three main economic benefits for simulating wind flow over complex terrain with the inclusion of wake effects. First, it allows analysis of wind speed and turbulence intensity at any location within the wind-field rather than relying on power law extrapolation from meteorological masts as discussed in Section 2.1. This allows for well informed decisions regarding turbine size and class. Secondly, it allows for experimental placement and optimization of wind farm layouts before any development takes place. Third, it can allow for power analysis to be completed of a wind farm with winds from various directions to create a better understanding of the production behavior of the farm. 


\subsection{Thesis Statement}

The research presented in this thesis focuses on two technical areas to advance the current state in wind power forecasting. The first is accurate simulation of wind flow over complex terrain using the immersed boundary (IB) method. The second is application of turbine wake models and development of a power estimation method for wind farms over complex terrain. The IB method is validated with two welldocumented test cases for atmospheric flow over complex terrain and the wake models are validated with wind tunnel and full scale field data.

\subsection{Work Published}

As part of this research, an article detailing our immersed boundary preprocessor for complex terrain was published in 2015 [57]. Another article detailing the methods we use to simulate winds over complex terrain and showing some of the associated sensitivities has been submitted for publication [16]. 


\section{CHAPTER 2}

\section{WIND PREDICTION OVER COMPLEX TERRAIN}

This chapter reviews the current practices for wind power forecasting and wind farm planning and addresses the most important variables in the process.

\subsection{Wind Resource Assessment}

Wind resource evaluation is a long and complex process that can be broken down into three main steps: site identification, resource monitoring, and a resource analysis [9]. These steps work from a large scale view and systematically narrow the siting down to the best wind turbine placements. Wind resource assessment is necessary due to the high variability of wind resources. There is variability over a large range of spatial and temporal scales [10]. This includes regional and climate variations, variation in complexity of terrain, year-to-year variations, and diurnal variations.

The preliminary site identification starts with collecting publicly available regional data such as airport wind data, local weather stations, and wind maps similar to Figure 2.1. The Wind Energy Resource Atlas of the United States [20] is a good resource for this. This information helps understand the available wind resources [34]. All of the collected data is interpolated to one consistent height and used to create a good picture of the large scale resources available. This data, along with other geographic data is compiled into a geographic information system (GIS). This allows 
the data to be analyzed based on the desired criteria and allows the user to find the best candidate sites. Other data, such as road access, transmission access, and community effects can be taken into account in the GIS [9].

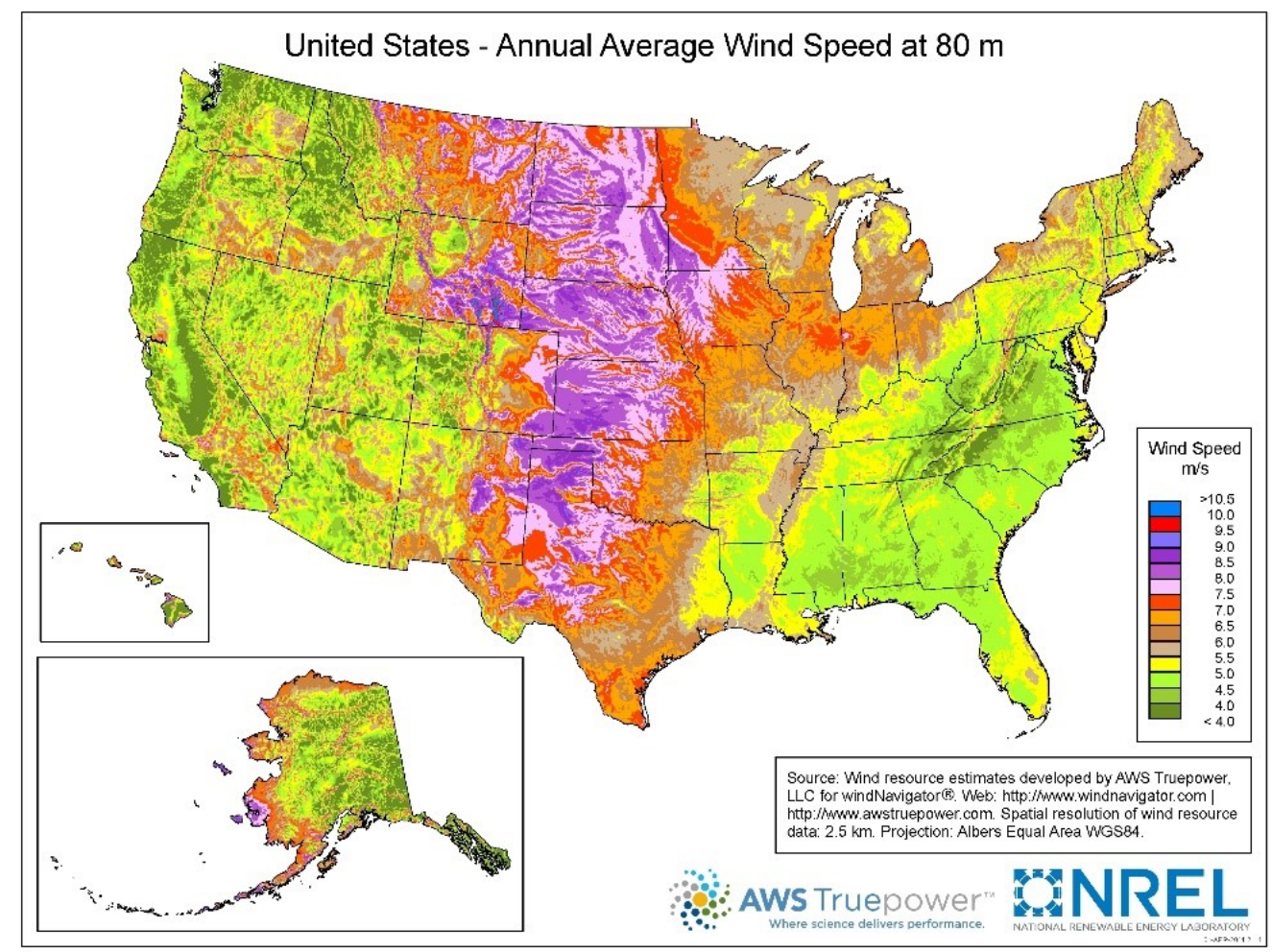

Figure 2.1: NREL wind map showing annual average wind speeds at 80 meters above ground level across the United States Figure is adopted from [30]. This map was created by the National Renewable Energy Laboratory for the U.S. Department of Energy with data provided by AWS TruePower.

Wind resource monitoring is the wind measurement campaign. One or more meteorological (met.) masts installed and measure wind speed, direction, and temperature. The masts need to be placed in such a manner that the engineers have a good understanding of the wind behavior in every direction. Larger projects (over $100 \mathrm{MW}$ ) require one third of the met masts be at hub height [9]. These campaigns 
need to last at least one year to get a good idea of seasonal and other time-scale effects. The effect of time-scales larger than one year can be extrapolated from historic surrounding wind data. On some projects, sonic detection and ranging (SODAR) and devices can be brought in for a shorter period of time to get a better idea of the shear profile. SODAR has only recently been utilized for wind resource assessment [34]. The processing of the massive amount of data from the measurement campaign is key to the assessment of the potential wind farm.

Resource analysis is the data processing and interpreting stage of wind siting. This includes data validation, wind resource characterization, estimation of the wind speed at hub height, uncertainty analysis, and sometimes use of CFD to better understand the wind site. All of this aids in optimal placement of turbines and an energy production estimate [9], which is the end goal wind resource evaluation.

The goal of a wind measurement campaign is to accurately represent the wind behavior in the potential plant site over the course of a year or more in order to appropriately size and place the turbines and forecast the power output of the proposed plant. Accurately characterizing the wind behavior with a few met masts comes with a set of challenges. Standard met masts do not come up to the turbine hub height, but wind resource assessment is evaluated at the hub height. This means that often the velocity must be extrapolated to the hub height. Usually the power law is used to extrapolate the data. Figure 2.2 compares extrapolations from a met mast to SODAR data and shows the large inconsistencies that can occur. SODAR is a much more accurate measuring tool, but is not the standard for wind measurement campaigns.

Other issues with met masts include that one or two masts may not be representative of the whole area. In a flat, relatively uniform site, one met mast may do a good 


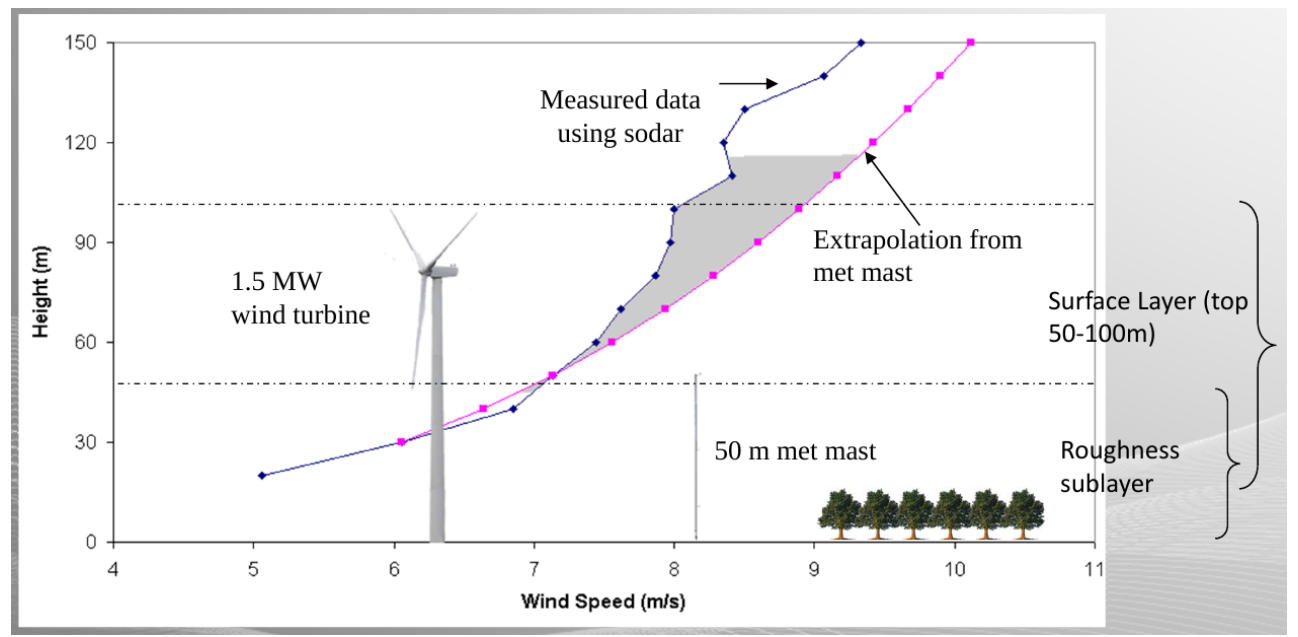

Figure 2.2: Observed difference between mast extrapolation and SODAR measurements. Image is adopted from AWS TruePower, a UL Company. [8]

job of characterizing the whole area. Inconsistencies in the roughness sublayer or terrain height can have a substantial effect on the velocity and cause inconsistencies in the calculated available power.

\subsection{Important Parameters}

The most important parameters with respect to wind farms are velocity, turbulence intensity, and wind shear. Velocity is the most important wind parameter in reference to wind power. The available power in the wind is proportional to the cube of the velocity. This means that wind speed has a drastic effect on the power that a turbine can extract from the air. That power is calculated from

$$
P_{\text {air }}=\frac{1}{2} \dot{m} U^{2}=\frac{1}{2} \rho A U^{3}
$$

where $P$ is available power, $\dot{m}$ is mass flow rate, $U$ is velocity, $\rho$ is density, and $A$ is swept area. With such sensitivity to velocity, a very accurate wind speed prediction 
at every turbine is vital. Extrapolating from low met. masts or trying to extrapolate a shear curve through complex terrain can lead to large errors and inaccurate power predictions.

Turbines come with power ratings and class ratings. These classes, as seen in Table 2.1, represent the conditions to which the turbine will be exposed. Turbulence intensity (TI), along with mean velocity are defining factors in deciding which class of turbine is appropriate for a site [9]. TI is defined as the "ratio of standard deviation of wind speed fluctuations to the mean" [10] and can be expressed as

$$
T I=\frac{u^{\prime}}{U}
$$

where $u^{\prime}$ is equal to the root-mean-square of the turbulent fluctuations. If TI is very large, the turbine might experience higher than expected velocity, meaning a safer class of turbine is necessary to avoid turbine failure. Collection of TI data from wind farms by either meteorological masts or turbine data is prone to significant errors [4]. Extreme 50 year gusts are another consideration when choosing the proper turbine. A 50 year gust is a gust of wind so severe that is unlikely to happen more than once every 50 years [10]. Turbines must be able to withstand these conditions.

Table 2.1: IEC 61400 standards for turbine classes. Table is adapted from [10].

\begin{tabular}{|l|l|l|l|l|}
\hline Wind Turbine Class & I & II & III & IV \\
\hline \begin{tabular}{l}
$V_{\text {ave }} \begin{array}{l}\text { average wind speed } \\
\text { at hub height }(\mathrm{m} / \mathrm{s})\end{array}$ \\
\hline $\begin{array}{l}V_{50} \text { extreme } 50 \text { year gust } \\
(\mathrm{m} / \mathrm{s})\end{array}$
\end{tabular} & 70.0 & 8.5 & 7.5 & 6.0 \\
\hline TI class A & 59.5 & 52.5 & 42.0 \\
\hline TI class B & \multicolumn{5}{|c|}{$18 \%$} \\
\hline
\end{tabular}

Engineers are very interested in the conditions that a turbine will be exposed to 
throughout its life. Wind shear, defined as the rate of change in wind speed along a vertical profile [9], can have a significant effect on large modern wind turbines. Since turbine diameters are continuing to increase in size in order to extract more energy from the wind, there is a large change in wind speed across the height of the rotor. This can be seen in the Figure 1.3. The velocity change can create a significant torque on the nacelle that can cause premature fatigue wear of parts.

\subsection{Turbine Siting}

Turbine placement is a large contributor to the complexities of wind power in complex terrain. Figure 2.3 shows an isocontour of the stream-wise velocity at $40 \mathrm{~m}$ above the surface in a simulation of Perdigao hill in Portugal simulated as part of this thesis research.

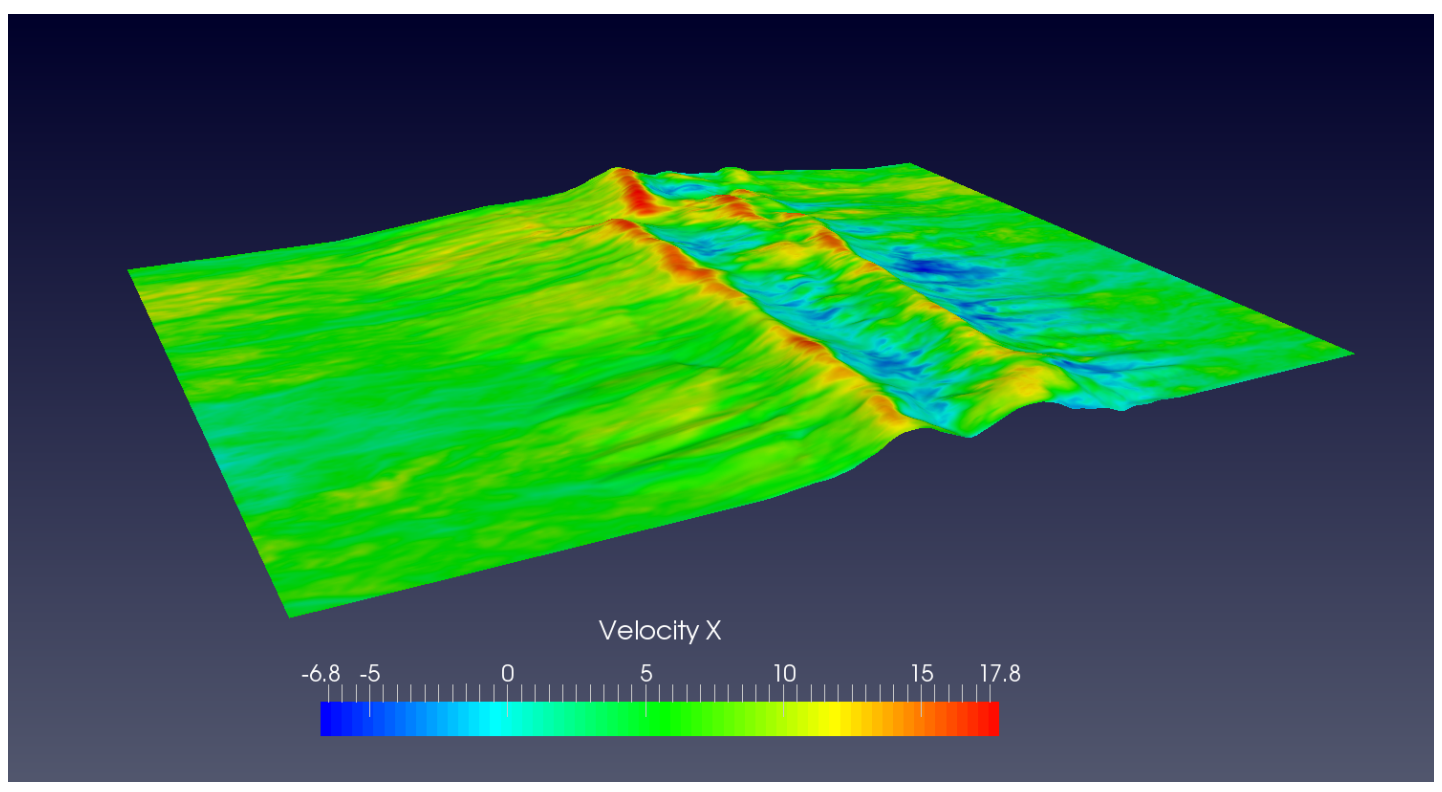

Figure 2.3: $40 \mathrm{~m}$ isosurface of velocity over Perdigao hill in Portugal.

It is evident that the terrain has caused complex wake effects both in between the 
hills and behind the second hill. A wind turbine could have a high power output, or much lower than expected output depending on its location. This is doubly important for wind power assessment in complex areas. Using one, two, or even five meteorological masts would still not be enough to accurately assess the wind behavior in locations similar to this one. The only currently feasible way to assess this location is to run an accurate terrain flow simulation. Furthermore, a terrain flow simulation with integrated wake models will give a detailed picture of the expected power output and wake effects from installing wind turbines in such a location.

\subsection{Wind Farm Design}

Selection of wind farm design technologies is one of the first steps in designing a wind farm and evaluating the power potential. These softwares include WindFarmer [24], WindFarm [32], WindPro [1], and openWind [66]. and contain features such as importing of data from sensors and simulations, turbine characterization and placement, estimation of energy production, and calculation of wake and other losses [9]. These softwares are designed to evaluate many characteristics of the wind farm, not just power and losses. As such, optional capabilities include uncertainty analysis, noise level calculations, analysis of local impact, and design of transportation and transmission access [9].

Impacts like visibility and noise creation are very important for wind farms. Development around areas of high sensitivity, such as residential land or recreational land, may not be acceptable [10]. When designing the farm, a wind resource analyst must consider four main goals: maximize use of land under developer's control, maximize average output as a fraction of rated capacity, minimize the installation cost of the development, and stay within regulations [9]. Many of the programs 
include a layout optimizer to maximize net energy output while acting within the set constraints $[9]$.

\subsection{Wind Power Forecasting}

As discussed earlier, wind forecasting is necessary due to the inherent variability of wind power production, and is currently done using some combination of statistical tools and NWPs. Statistical methods are generally used for very short term forecasts, whereas climatological models like NWPs are better for forecasting wind speed at more than 15 hours [23]. The timescales of interest when considering wind forecasts for power generation and farm operation are the very short term of under one minute, the short term of less than two days, and the medium term of 2-7 days [23]. This allows for planning of power commitment and various types of maintenance. Foley et al. discusses the current methods of wind power forecasting and predicts that continued wind energy development will drive and necessitate improvements in forecasting methods [23].

Cheng et al. showed some improvement to NWP models for wind speed prediction by assimilating anemometer data [13]. A couple of issues with these methods are that a small error in velocity can show large errors in wind power due to the nonlinearity of the turbine power curves and resource assessment becomes difficult due to the variation in environmental conditions [23]. Additionally, they do not consider the effects of turbine wakes.

\subsection{Current State of Wind Simulations}

Due to the enumerable detailed approaches to solving the N-S equations, there is a lot of variation in predictions of wind velocity over complex terrain. The Bolund 
Experiment $[6,7]$ is an example of the discrepancies that can be observed. Bolund Hill is surrounded almost entirely by water off the coast of Denmark near Denmark Technical University (DTU). The hill displays a very sharp escarpment on its front face. The Bolund Experiment was a detailed campaign to capture the flow over the hill at 8 separate locations and multiple vertical heights. It has since become a very challenging case used to validate CFD codes' abilities to capture the complex flow behavior.

As part of the Bolund Experiment, 57 different participants from research universities, wind energy consulting, turbine manufacturing, and CFD development were given the same environmental conditions and asked to simulate the flow over the hill. Their results are shown in Figure 2.4. The results show a great deal of variation and very few are able to capture the velocity at all of the mast locations. This displays the incredible importance of sensitivity studies and validation. Parameters such as boundary conditions and mesh resolution can have drastic effects on the outcome of a simulation. As part of this thesis research, GIN3D is validated using the Bolund Hill case and the Askervein (Scotland) case with the goal of increased accuracy in wind velocity predictions over complex terrain. 


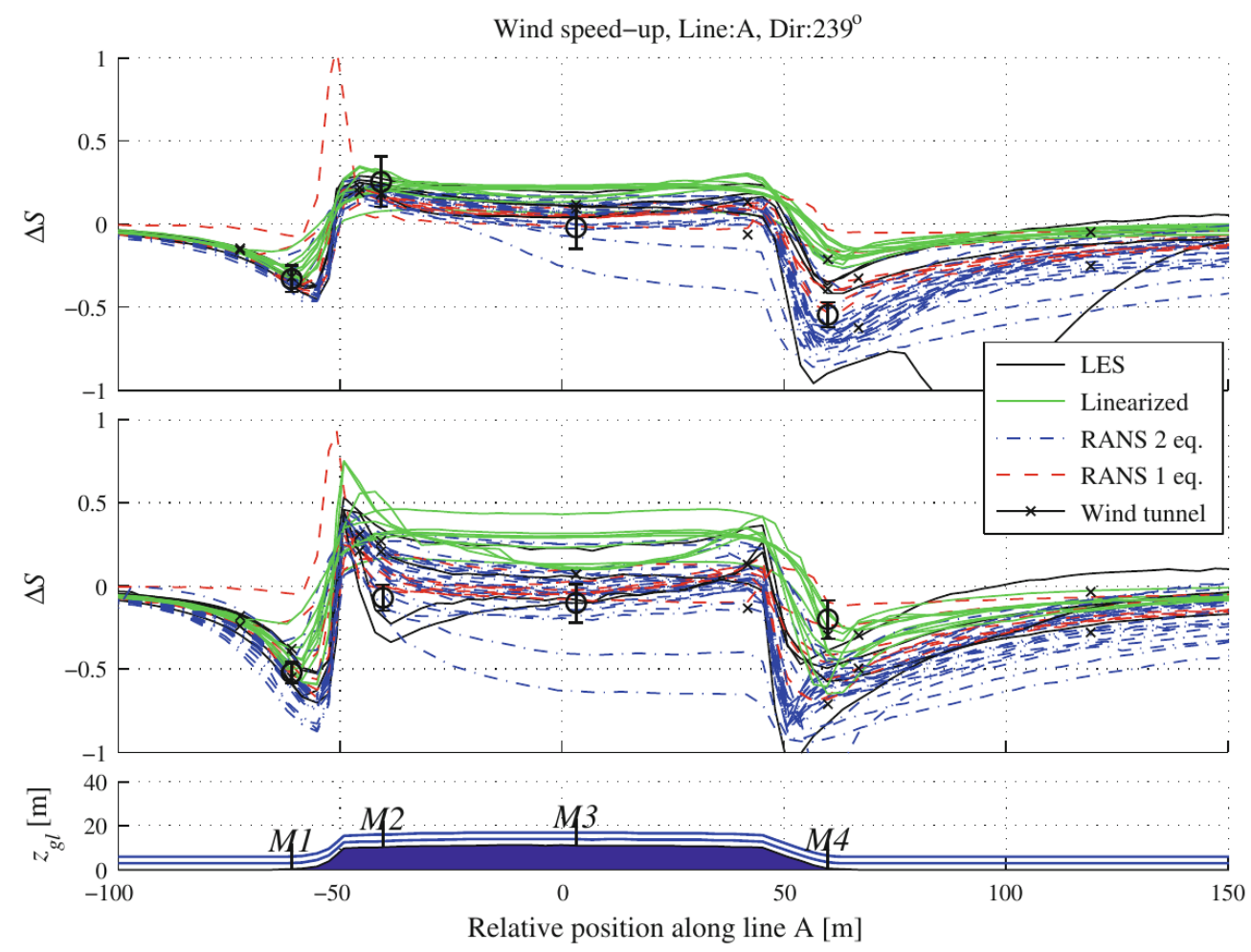

Figure 2.4: Example of variation in fractional speedup, $\Delta S$, along line A from the Bolund Blind Study results. Profiles are given at $2 \mathrm{~m}$ and $5 \mathrm{~m}$ above the hill. Plot is adopted from Bechmann et al. [6]. 


\section{CHAPTER 3}

\section{TECHNICAL BACKGROUND}

This chapter presents the governing equations and the key numerical methods used in the wind solver. All computations are performed in parallel on multiple GPUs on a high performance computing cluster to decrease run times and move towards forecasting of wind flow and wind power.

\subsection{Immersed Boundary Method}

The immersed-boundary (IB) method was originally proposed to simulate human heart valves [46]. The method gained popularity [41] after the previous stability issues were addressed with the introduction of the direct forcing method [22, 42]. For the complex geometry, the body force is implicitly included by reconstruction at the cells that are cut by the terrain. The IB method has proven successful in resolving terrain and complex geometry. It can also be implemented in existing flow solvers without modifications to the core flow solver [57].

The IB method works by immersing a geometry (the immersed boundary) in a structured Cartesian grid. The Cartesian grid points, or nodes, are marked according to their relation to the geometry. The nodes within the geometry are marked as solid, the nodes exterior to the geometry are marked as fluids, and the exterior nodes adjacent to the geometry are labeled as IB nodes. The node labeled $I B$ in Figure 3.1 


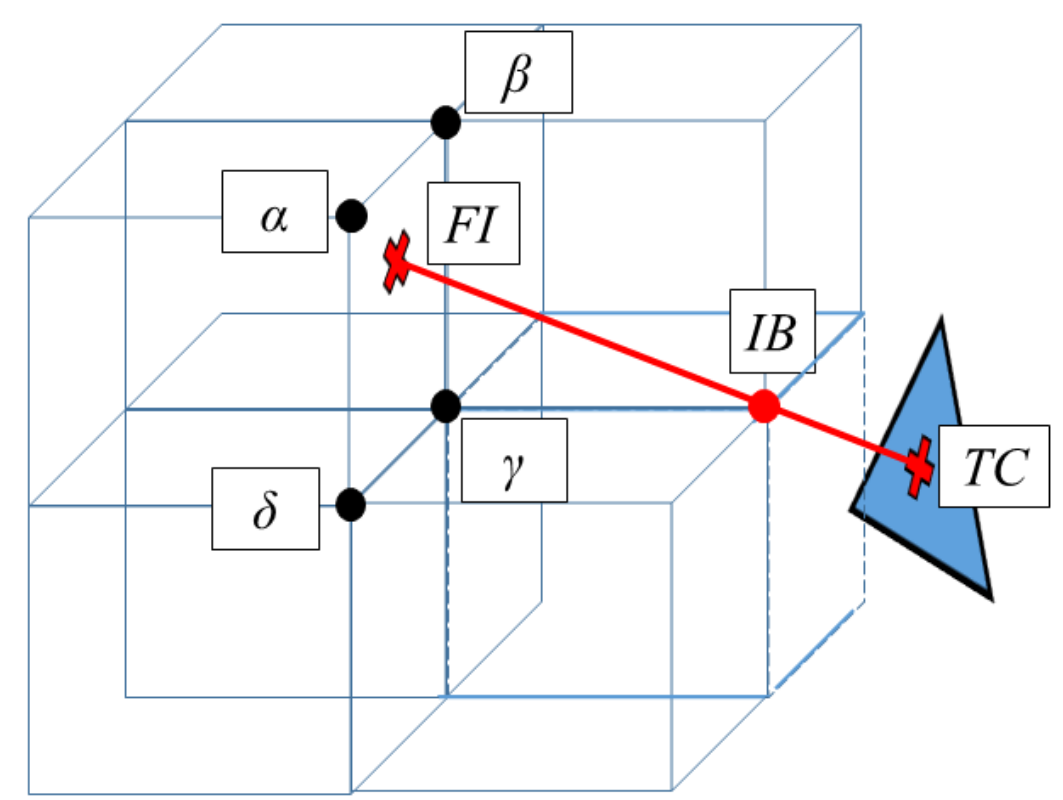

Figure 3.1: IB reconstruction in the surface normal direction. $\alpha, \beta, \gamma$, and $\delta$ are the Cartesian nodes used to interpolate onto $F I$. $T C$ is the triangle centroid, $I B$ is the immersed boundary node, and $F I$ is the cell face intersection. Interpolation occurs along the projected line from $T C$, through $I B$, to $F I$.

represents an IB node.

The implementation of the direct forcing approach to the IB method $[22,42]$ is based on solving the the discretized governing momentum equation,

$$
\frac{u_{i}^{t+1}-u_{i}^{t}}{\Delta t}=R H S_{i}^{t}+f_{i}^{t}
$$

for the body forcing term $f^{t}$ such that $u_{i}^{t+1}=u_{i, b c}^{t+1} \cdot u_{i, b c}$ is the velocity boundary condition which is known for all time-steps and $u^{t}$ represents the velocity at times-step t. RHS includes the convective, pressure gradient, and viscous terms. Rearranging the terms and ensuring that the forcing term produces the correct velocity at the boundary enables $f^{t}$ to be written as 


$$
f_{i}^{t}=-R H S_{i}^{t}+\frac{u_{b c}^{t+1}-u_{i}^{t+1}}{\Delta t}
$$

Because we use a Cartesian grid system, $u_{b c}$ may not align with the grid points. As such, it is necessary to have an interpolation scheme to reconstruct the velocity at the near-surface grid points and enforce the surface boundary condition. In order to reconstruct the velocity field near the surface, we adopt the log-law IB reconstruction proposed by Senocak et al. for rough, flat terrain [56]. They proposed a logarithmic reconstruction for velocity tangent to the surface and a linear reconstruction for velocity normal to the surface. The latter enforces the impermeability condition. To show the reconstruction on velocity, we start with the rough log-law [60],

$$
\phi=\frac{u_{*}}{\kappa} \ln \left(\frac{h+z_{0}}{z_{0}}\right) .
$$

In this equation, the variable $\phi$ will represent wind speed, $u_{*}$ is the friction velocity, $\kappa$ is the von Kármán constant, $h$ is the normal elevation from the surface, and $z_{0}$ is the aerodynamic roughness length. We assume that friction velocity is held constant and divide the log-law at heights $h=h_{I B}$ and $h=h_{F I}$ from Figure 3.1 to get the relationship

$$
\frac{\phi_{I B}}{\phi_{F I}}=\frac{\frac{u_{*}}{\kappa} \ln \left(\frac{h_{I B}+z_{0}}{z_{0}}\right)}{\frac{u_{*}}{\kappa} \ln \left(\frac{h_{F I}+z_{0}}{z_{0}}\right)} .
$$

The logarithmic reconstruction scheme for the tangential velocity simplifies to

$$
\phi_{I B}=\phi_{F I} \frac{\ln \left(\frac{h_{I B}+z_{0}}{z_{0}}\right)}{\ln \left(\frac{h_{F I}+z_{0}}{z_{0}}\right)},
$$

where $\phi$ represents the wind speed tangential to the surface. $\phi_{F I}$ is calculated using 
bilinear interpolation from nodes $\alpha, \beta, \gamma$, and $\delta$ pictured in Figure 3.1. The normal component of velocity is reconstructed using linear interpolation between $F I$ and $T C$ in Figure 3.1 as

$$
\phi_{I B}=\frac{h_{I B}}{h_{F I}} \phi_{F I}+\phi_{T C}
$$

where $\phi$ represents the wind speed normal to the surface. From the no-slip condition, $\phi_{T C}=0$ is enforced. $\phi_{F I}$ is the same value from Equation 3.5. After the logarithmic and linear projections are completed, the results are formed into a vector in the same direction as the flow at point FI. It is assumed that the flow direction stays constant in the surface normal direction in close proximity of the surface. Roman et al. has a similar scheme, breaking the velocity at the IB node into tangential and normal components and reconstructing them using different methods for simulation of turbulent channel flow [53]. In their case, a smooth-wall log law was used for the tangential component and the normal component was reconstructed using a quadratic polynomial.

The advantage of the IB method for complex terrain is twofold. First, the mesh generation over the large, complex terrain is automated and rapid. Second, flow solutions are much quicker on a structured mesh such as the Cartesian grid used by the IB method than computations using an unstructured mesh. The speed and ease of use is imperative when trying to acquire real time solutions to simulations such as wind power predictions and contaminant spread predictions.

We developed an IB preprocessor to generate the geometric data to implement the IB method [57]. The preprocessor is used to tag nodes, calculate the distance from an IB node to the surface for use in reconstruction, and bind an IB node 
with a surface element. Terrain maps are obtained from the U.S. Geological Survey website. These maps are then converted to Digital Elevation Maps (DEMs) using MICRODEM [27] and fit with a triangulated surface mesh using a function MATLAB Central File Exchange [37]. The triangulated geometries can then be saved in the stereolithography(STL) file format for use in the IB preprocessor software.

The IB preprocessor software calculates which Cartesian nodes are inside and outside of the geometry, and tags the nodes directly on the outside of the surface as IB nodes. The IB preprocessor computes the distance field for complex terrain by solving the Eikonal equation using the fast sweeping method of Zhao [78]. A visualization of the distance field for a section of Hells Canyon can be seen in Figure 3.2 .

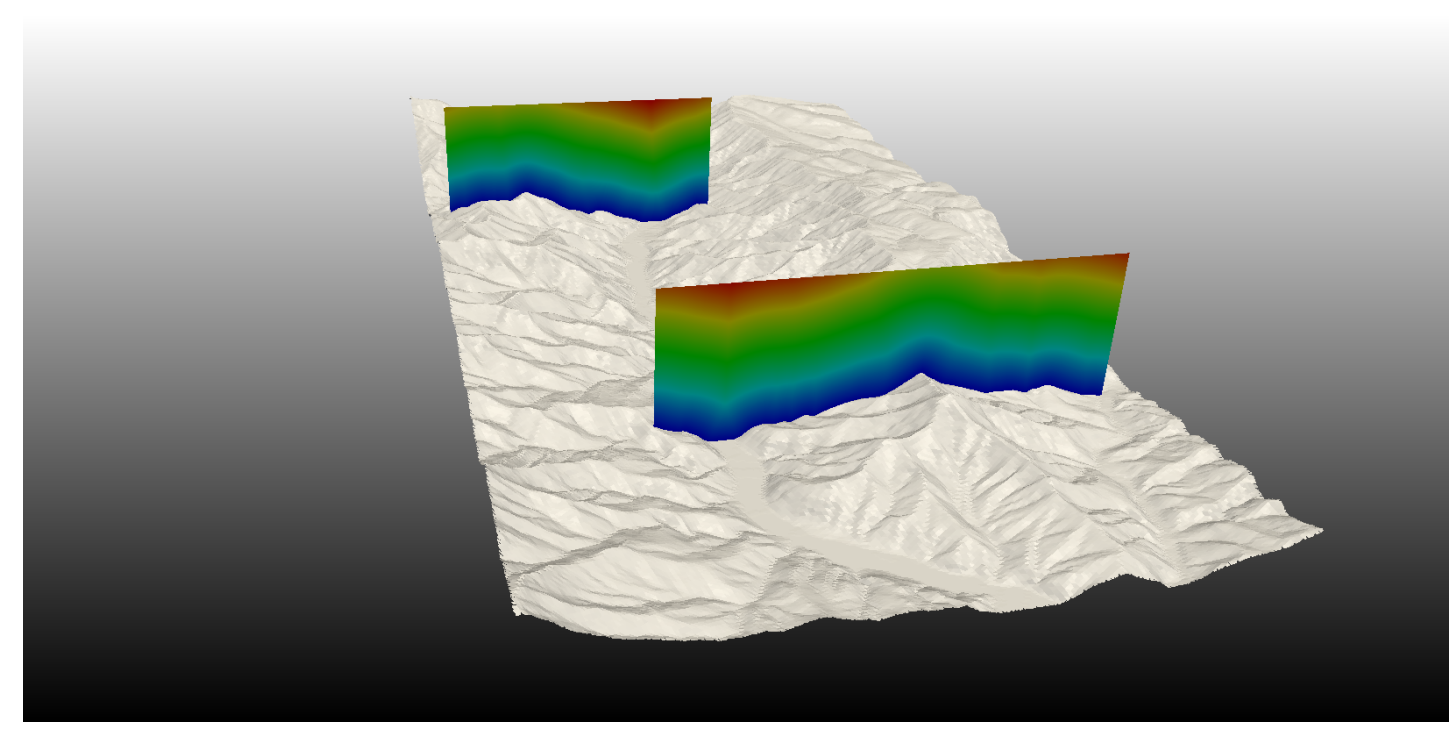

Figure 3.2: Visualization of the distance field from the complex terrain of Hells Canyon, Idaho. 


\subsection{Governing Equations}

The incompressible form of the Navier-Stokes (N-S) equations are numerically solved in the GIN3D flow solver. Figure 3.3 depicts the expected energy spectrum for turbulent flows.

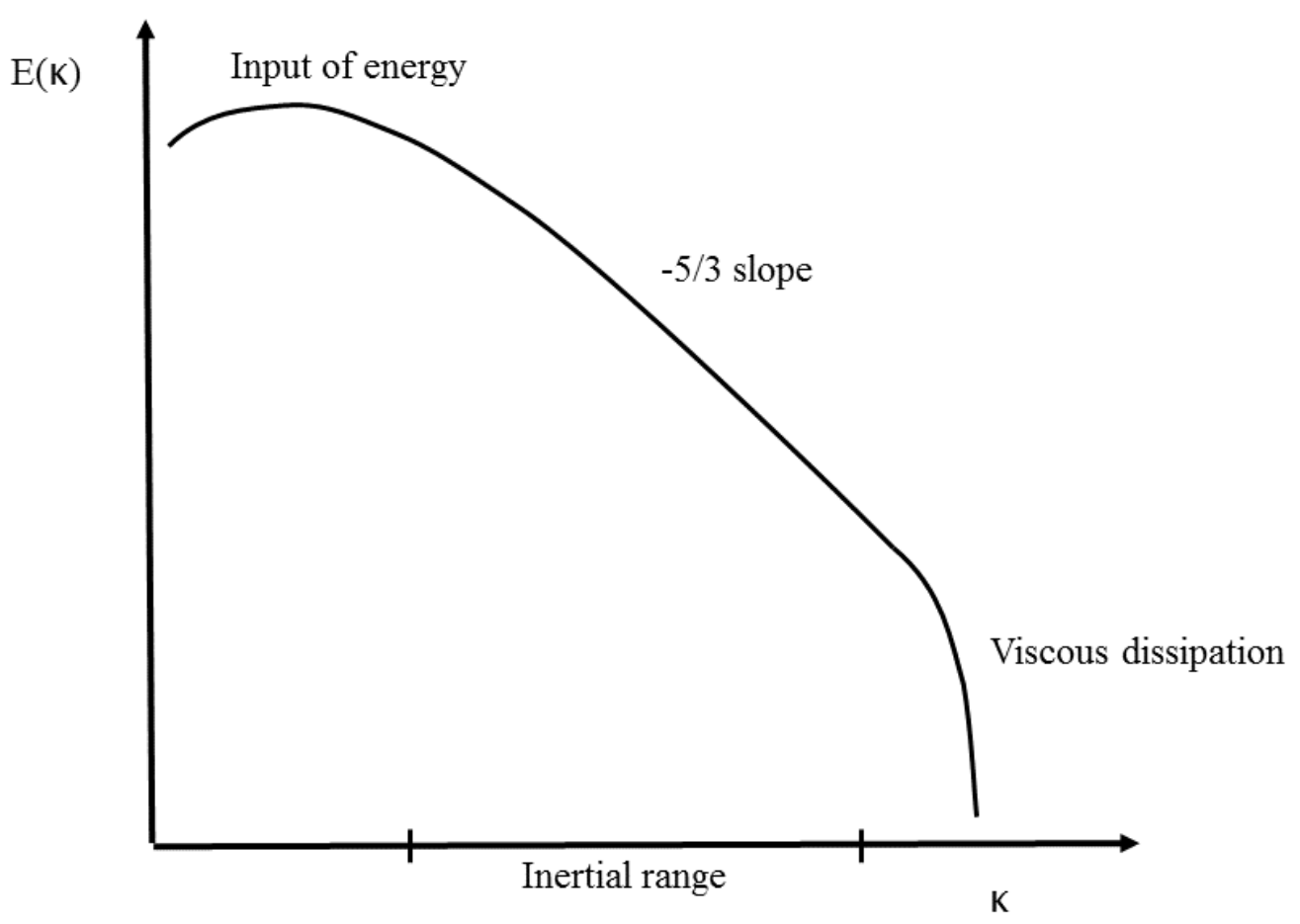

Figure 3.3: Schematic of the energy spectra for turbulent flows.

Numerically, turbulence is addressed by three major techniques. Direct Numerical Simulation (DNS) resolves all energy scales and is computationally expensive and limited to a few fundamental flow problems. In contrast, Reynolds Averaged Navier Stokes (RANS) only resolves the eddies at the integral length scale. RANS treats parameters as a mean plus a fluctuation about the mean. RANS is less detailed than LES in terms of flow structures, but requires less grid resolution near walls and is less computationally expensive. 
LES filters out everything passed the filter size and approximates the effects of the viscous dissipation on the larger scales using the subgrid-scale (SGS) stress tensor. We use hybrid RANS-LES [55] in order to parameterize turbulence in the vicinity of the surface to obtain accurate wind predictions. RANS is used to simulate the flow nearest to solid walls without requiring any adaptive mesh refinement, while LES is used to accurately simulate the turbulent eddies everywhere else in the domain. This allows us to obtain a decent accuracy at an affordable computational expense.

The LES form of these equations appear in their filtered form, with the over-bar indicating a filtered value. The filter width is given as $\Delta=\sqrt[3]{d x \cdot d y \cdot d z}$, where $d x$, $d y$, and $d z$ are the cell sizes in the $x, y$, and $z$ directions. LES involves spatially filtering rather than time-averaging. The effects of the small eddies on the larger resolved eddies can be parameterized, while the eddies operating at a scale larger than the filter width can be simulated. The momentum and mass equations are

$$
\begin{aligned}
\frac{\partial\left(\rho \bar{u}_{j}\right)}{\partial x_{j}} & =0 \\
\frac{\partial\left(\bar{u}_{i}\right)}{\partial t}+\frac{\partial\left(\bar{u}_{i} \bar{u}_{j}\right)}{\partial x_{j}} & =-\frac{1}{\rho} \frac{\partial \bar{p}}{\partial x_{i}}-\frac{f_{i}}{\rho}+\frac{\partial}{\partial x_{j}}\left(2 \nu \bar{S}_{i j}-\tau_{i j}\right),
\end{aligned}
$$

where $u$ is the velocity vector, $x$ is the position vector, $p$ is pressure, $\rho$ is density $\bar{S}_{i j}$ is the strain rate tensor and $\tau_{i j}$ are the filtered Reynolds stresses. The induced turbine forces can be included as body-forces, $f_{i}$, which is discussed later in Section 4.1. The Reynolds stresses are modeled using an eddy-viscosity model in Equation 3.9 and Equation 3.10.

$$
\begin{aligned}
\tau_{i j} & =\nu_{t} \bar{S}_{i j}, \\
\nu_{t} & =l_{m i x}^{2}|\bar{S}| .
\end{aligned}
$$


For the hybrid RANS-LES model we use the near-surface model as in [55], which blends the LES length scale with the Prandtl mixing length scale [51] such that $l_{\text {mix }}$ in 3.10 becomes,

$$
l_{\text {mix }}=\left(1-\exp \left(-z_{n} / h_{B}\right)\right) C_{S} \Delta+\exp \left(-z_{n} / h_{B}\right) \kappa h .
$$

In this equation, $z_{n}$ is the normal distance from the terrain, $h_{B}$ is the RANS-LES blending height, and the blending height is $\Delta=\sqrt[3]{d x \cdot d y \cdot d z}$. The Smagorinsky coefficient, $C_{S}$, is determined using the localized Lagrangian dynamic Smagorinsky SGS model [38]. At IB Cartesian nodes close to the surface, the RANS-LES model will be dominated by the RANS approximation, which means the Prandtl mixing length model will be used for the SGS model.

\subsection{Numerical Methods}

The governing equations, equations 3.7 and 3.8, are solved using a second-order Adams-Bashforth scheme for time advancement. We use a second-order central difference scheme to discretize the spatial derivatives on a directionally uniform Cartesian grid. The pressure Poisson equation was solved using an amalgamated parallel geometric multigrid technique [28]. The 3D incompressible flow solver is executed in parallel on a graphical processing unit (GPU) cluster [15, 28, 65]. 


\section{CHAPTER 4}

\section{WIND FARM MODELING}

This section explains the turbine wake models that enable the introduction of wake effects into the wind simulations to model an entire wind farm. Single turbines and wind farms with multiple turbines are simulated in a variety of environments, which enables us to study wake-wake interactions. Additionally, this section covers a scalable energy analysis method for assessment of turbine power in complex terrain and wake power losses in wind farms.

\subsection{Wind Turbine Wake Models}

In order to simulate an entire wind farm, it has become common practice to parameterize turbine-induced forces using actuator disk models [68]. These models enforce turbine forces such as lift and drag without resolving the boundary layer flow around the turbine blades. The rotational models come from the Blade Element/Momentum (BEM) method $[25,34]$ and assume that the blades can be divided into independent elements in order to parameterize their forces [58].

\subsubsection{Actuator Disk Model with No Rotation}

The actuator disk model with no rotation (ADM-NR) models the turbine rotor as a disk that applies an axial force on the air based on a thrust coefficient. The thrust 
coefficient, $C_{T}$, is dimensionless and can be found from turbine power and thrust curves or integrating the change in lift and drag forces over the blade length [71]. A power curve for the Suzlon S88 turbine is presented in Figure 4.1.

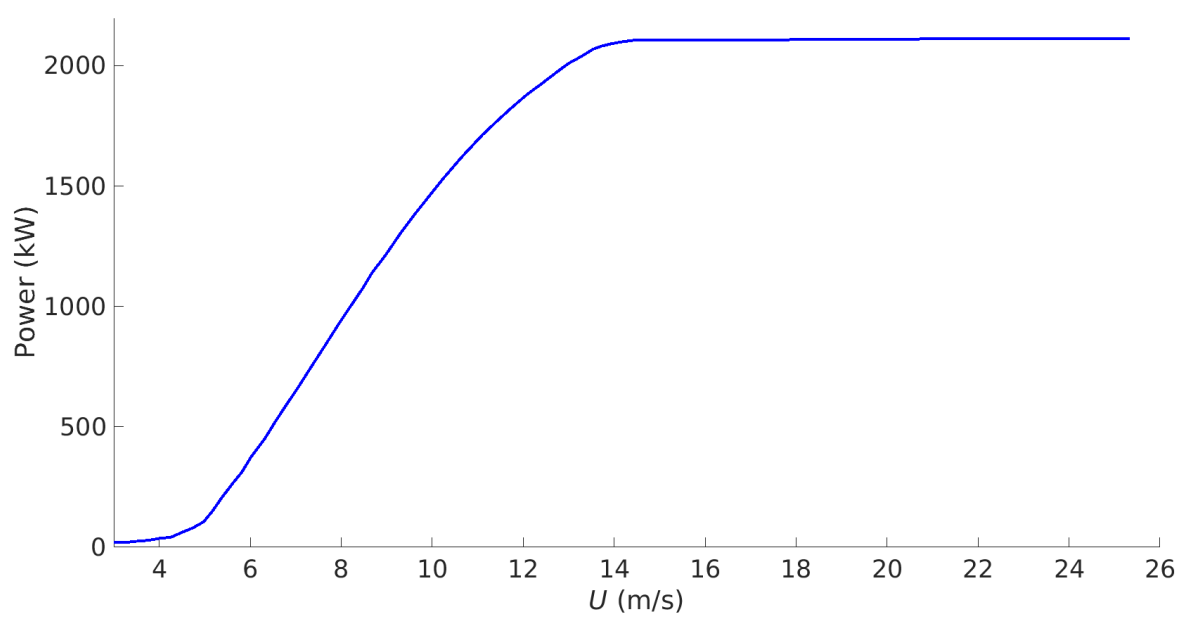

Figure 4.1: Plot of power as a function of wind speed for the Suzlon S88-2.1 MW wind turbine. Data is from [62].

If the thrust curve is not given, the thrust coefficient can be calculated from the axial induction factor, $a$, by $C_{T}=4 a(1-a)$. The axial induction factor is defined as the fractional decrease in velocity from the free-stream to the rotor plane [10, 34]. A visualization of the relationship between $a$ and incoming velocity can be seen in Figure 4.2, and a generic plot showing the relationship between $a, C_{T}$, and $C_{P}$ is shown in Figure 4.3. The axial induction factor can be calculated from its relationship with the coefficient of power $C_{P}=4 a(1-a)^{2}$ and must be between 0 and $1 / 3$ for the power coefficient to stay below the theoretical Betz limit of 0.5926 [34]. The equation for the total $1 \mathrm{D}$ axial force caused by the turbine is given by

$$
F_{x}=\frac{1}{2} \rho U^{2} A C_{T}
$$


where $U$ is the upstream, streamwise velocity at the rotor hub height sampled one rotor diameter (D) upstream, $A$ is the rotor area, and $\rho$ is the density. Studies on application of the ADM-NR shows good agreement with velocity profiles in the far wake region, but poor performance as compared to other models in the near wake region $[50,59,71]$. It also does not capture the complicated flow structures such as tip vortices, rotation, and asymmetry of the wake. This is a simple model to apply, but does not provide as complete characterization of wake behavior as other wake models.

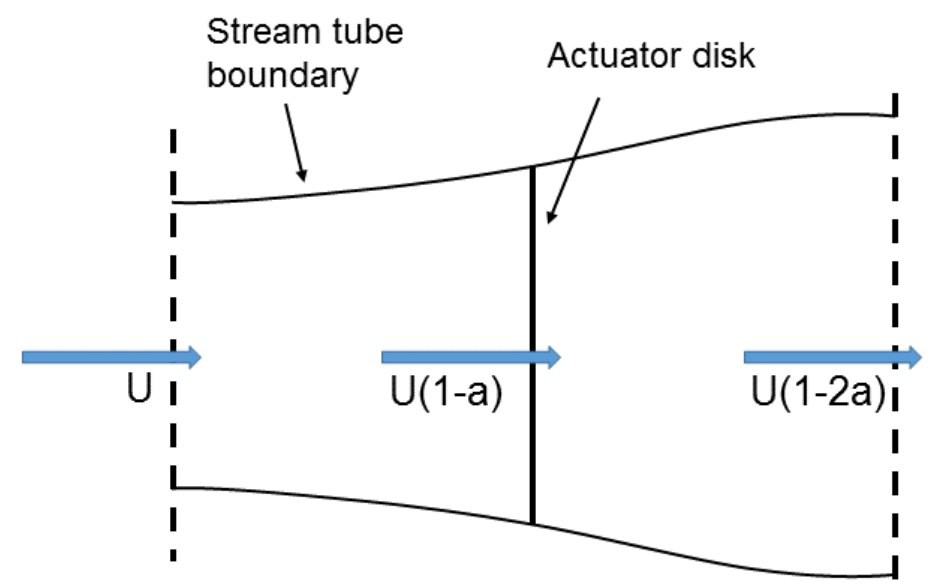

Figure 4.2: Visualization of the stream tube boundary around the actuator disk showing the fractional relationship between incoming velocity and the axial induction factor. Image is adapted from [34].

\subsubsection{Actuator Disk Model with Rotation}

Another common model that builds from the ADM-NR is the actuator disk model with rotation (ADM-R) [34, 50, 71, 72]. This model takes into account the drag and lift caused by the blades and distributes the force over a disk shape by use of BEM. 


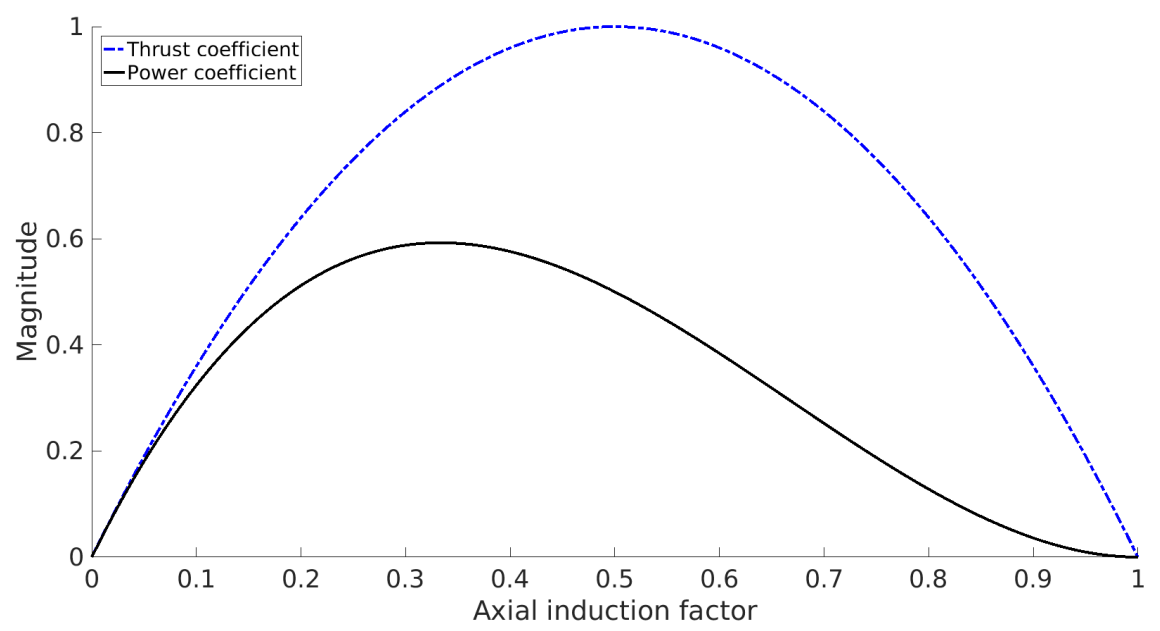

Figure 4.3: Plot showing the relationship between $a$ and the thrust and power coefficients. Image is adapted from [34].

The ADM-R takes into account the induced flow rotation and the nonuniform force distribution over the rotor face. The equation for the force per area of an annular segment is

$$
\boldsymbol{f}_{2 D}=\frac{d \boldsymbol{F}}{d A}=\frac{\rho U_{r e l}^{2}}{2} \frac{B c}{2 \pi r}\left(C_{L} \boldsymbol{e}_{\boldsymbol{L}}+C_{D} \boldsymbol{e}_{\boldsymbol{D}}\right)
$$

where $d A$ for the annular segment is equal to $2 \pi r d r$. This model uses the lift and drag coefficients of the blades and takes into account the chord length variation along the blades. $r$ is the radial coordinate of the blade section, $B$ is the number of blades, $c$ is local chord length of the airfoil, $C_{L}$ and $C_{D}$ are the lift and drag coefficients that depend on the angle of attack, $\alpha$, and the Reynolds number, and $\boldsymbol{e}_{\boldsymbol{L}}$ and $\boldsymbol{e}_{\boldsymbol{D}}$ are the unit vectors in the lift and drag directions. $U_{r e l}$ is the local relative velocity to the blade and is calculated by

$$
U_{r e l}=\frac{U(1-a)}{\sin (\phi)}
$$


as in [34]. The angle between $U_{r e l}$ and the rotor plane, $\phi$ is calculated by

$$
\phi=\tan ^{-1}\left(\frac{1-a}{\left(1+a^{\prime}\right) \lambda_{r}}\right)
$$

where $\lambda_{r}$ is the local tip speed,

$$
\lambda_{r}=\frac{\lambda r}{R}
$$

and $a^{\prime}$ is the tangential induction factor. The angle of attack is calculated from $\alpha=\phi-\gamma$, where $\gamma$ is the local pitch angle of the annular segment. The drag and rotational forces for an annular ring in the rotor plane are

$$
\begin{aligned}
F_{\theta} & =\frac{1}{2} B c U_{r e l}^{2}\left(C_{L} \sin (\phi)-C_{D} \cos (\phi)\right) d r \\
F_{X} & =\frac{1}{2} B c U_{r e l}^{2}\left(C_{L} \cos (\phi)+C_{D} \sin (\phi)\right) d r .
\end{aligned}
$$

A visualization of an airfoil cross-section is shown in Figure 4.4. The airfoil data are generally obtained from wind tunnel experiments and corrected for 3D effect [58]. One example of this procedure is given in [71]. The force components in the lift and drag directions relative to the blade can be projected onto the normal and rotational directions relative to the rotor [34] and then onto the Cartesian $x, y$, and $z$ directions. Details on the development of this model based on BEM are explained in [34]. The second iterative method presented for BEM by Manwell is used to solve for $a, a^{\prime}, C_{L}$, and $C_{D}$ [34]. A visualization showing the blade element mesh overlaid on a Cartesian mesh is shown in Figure 4.5. This model does not capture the tip and root vortices, but it accurately models the velocity drop in the near and far wake region better than 
the ADM-NR [50, 71].

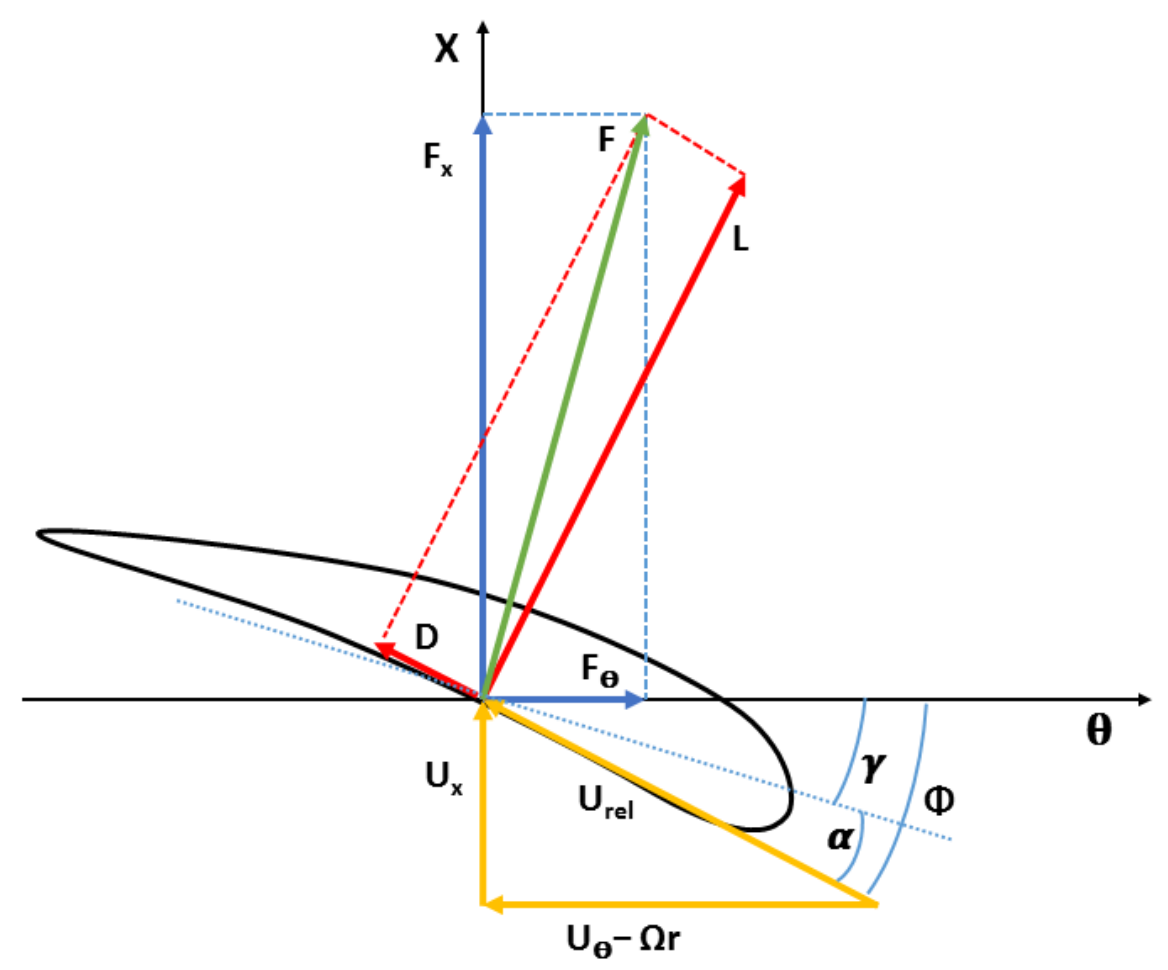

Figure 4.4: Visualization of an airfoil cross-section showing the relative forces, angles, and velocities for the BEM approach. $\gamma$ is the local pitch angle, $\alpha$ is the angle of attack, and $\phi$ is the angle of relative wind at which $U_{r e l}$ approaches. Image is adapted from [50].

\subsubsection{Actuator Line Model}

The Actuator Line Model (ALM), developed by Sørensen and Shen, is a wake model that relies on the same tabulated airfoil data and BEM equations as the ADM$\mathrm{R}$, but does not spread the forces over a disk [58]. Instead, the ALM distributes the 


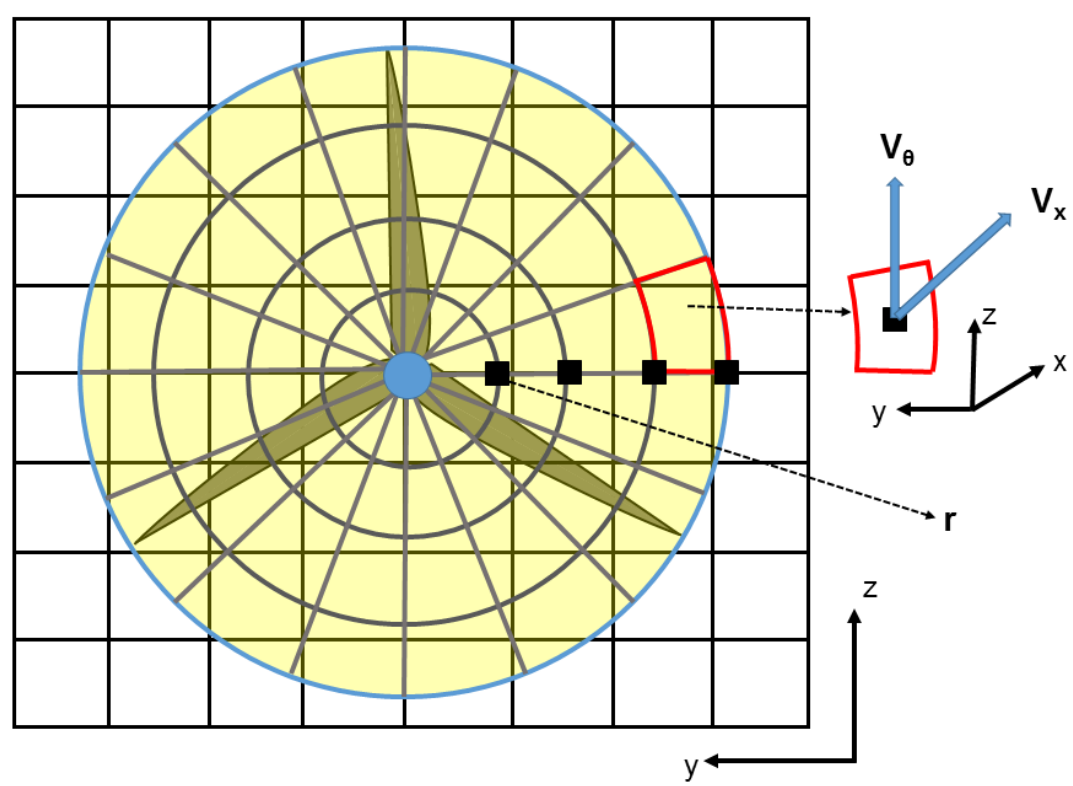

Figure 4.5: Visualization the blade element mesh overlaid on the Cartesian mesh. $V_{x}$ and $V_{\theta}$ are the axial and rotational components of the velocity, the uses of which are detailed in [34]. $r$ is the radius at each blade element. Image is adapted from [72].

aerodynamic forces along lines that represent the turbine blades. The equation for force acting along the lines becomes

$$
\boldsymbol{f}_{1 D}=\frac{d \boldsymbol{F}}{d r}=\frac{\rho U_{r e l}^{2} c}{2}\left(C_{L} \boldsymbol{e}_{\boldsymbol{L}}+C_{D} \boldsymbol{e}_{\boldsymbol{D}}\right)
$$

This model is able to capture the complex flow structures and the velocity drops quite well [50]. The ALM can exhibit run times similar to a fully resolved rotor simulation due to time-step restrictions required to track the rotation of the actuator lines [40]. Additionally, it shows very similar wake velocity profiles [50] and power predictions [36] to the ADM-R. For these reason, we have chosen to focus on the application of the actuator disk models for this thesis. 


\subsection{Computer Implementation}

When applying any of the turbine wake models to a CFD code, care must be given to avoid numerical instabilities. The body force is calculated using a Gaussian distribution kernel to smear the force onto the Cartesian grid points [36, 39]. This is done by taking a convolution integral over the turbine area and a tunable distribution width as in [39]. The formulation is used to make sure that the overall force is conserved and that there are no singularities. For the ADM-NR, we use the 1D regularization kernel,

$$
\eta_{\epsilon}=\frac{1}{\epsilon \sqrt{\pi}} e^{-\left(\frac{p}{\epsilon}\right)^{2}}
$$

This method takes the evenly distributed load on the disk and spreads it in the streamwise direction. The kernel is applied such that the body force at an individual node is

$$
\boldsymbol{f}=\frac{1}{\Delta v} \boldsymbol{F} \otimes \eta_{\epsilon}=\frac{1}{\Delta v} \int_{-\infty}^{\infty} \frac{\boldsymbol{F}}{\epsilon \sqrt{\pi}} e^{-\left(\frac{p}{\epsilon}\right)^{2}} d n .
$$

In this formulation $\otimes$ represents a convolution, $\Delta v$ is the volume of a cell, $p$ is the stream-wise distance from the rotor to the point being calculated, $n$ is the normal direction away from the rotor, and $\epsilon$ adjusts the distribution and is equal to a multiple of the stream wise grid spacing. For the ADM-R, we use the 3D regularization kernel,

$$
\eta_{\epsilon}=\frac{1}{\epsilon^{3} \pi^{3 / 2}} e^{-\left(\frac{p}{\epsilon}\right)^{2}}
$$

as applied in [39]. In this form, $p$ becomes the 3D distance from the rotor element to the Cartesian point. The 3D distribution kernel takes the force applied to the 
blade elements and smears it over the prescribed volume. Martínez-Tossas et al. explored the sensitivity of the ADM and ALM to mesh size and distribution width [36]. They found major sensitivity in predicted power output from both parameters and sensitivity in the wake velocity profiles based on the relationship between the streamwise grid resolution and the distribution width.

Along with simulating the rotor force, the turbine nacelle can be simulated as a bluff body. As such, a standard drag model can be applied with an assigned coefficient of drag, $C_{D}$. The force in the streamwise direction is then given as $F_{x}=\frac{1}{2} \rho U^{2} A C_{D}$. In our cases, the nacelle is approximated as a cylinder [43] with $C_{D}$ equal to 0.85 , as in [71]. Mittal et al. investigated the effects of including the nacelle in wake model simulations and concluded that there are definite improvements in the wake velocity profiles [40].

Once the force is calculated and the regularization kernel is applied to prevent any instabilities, the force can be treated as a body force at the specific grid points. The force is applied to the N-S equations through the body forcing term in the momentum balance Equation (3.8). Pseudo-code for the ADM-NR and ADM-R implementation is shown in Algorithm 4.1 and Algorithms 4.2 and 4.3. Implementation of the ADM-R can vary based on available blade data, and is discussed in more detail for each case in section 5.2 .

\subsection{Power Output Estimation}

Currently, there is not an established method for calculation of wind power output. Common methods are listed in Table 4.1, along with their advantages and disadvantages. The common methods often require detailed knowledge of wind turbine operation within the environment of interest. 


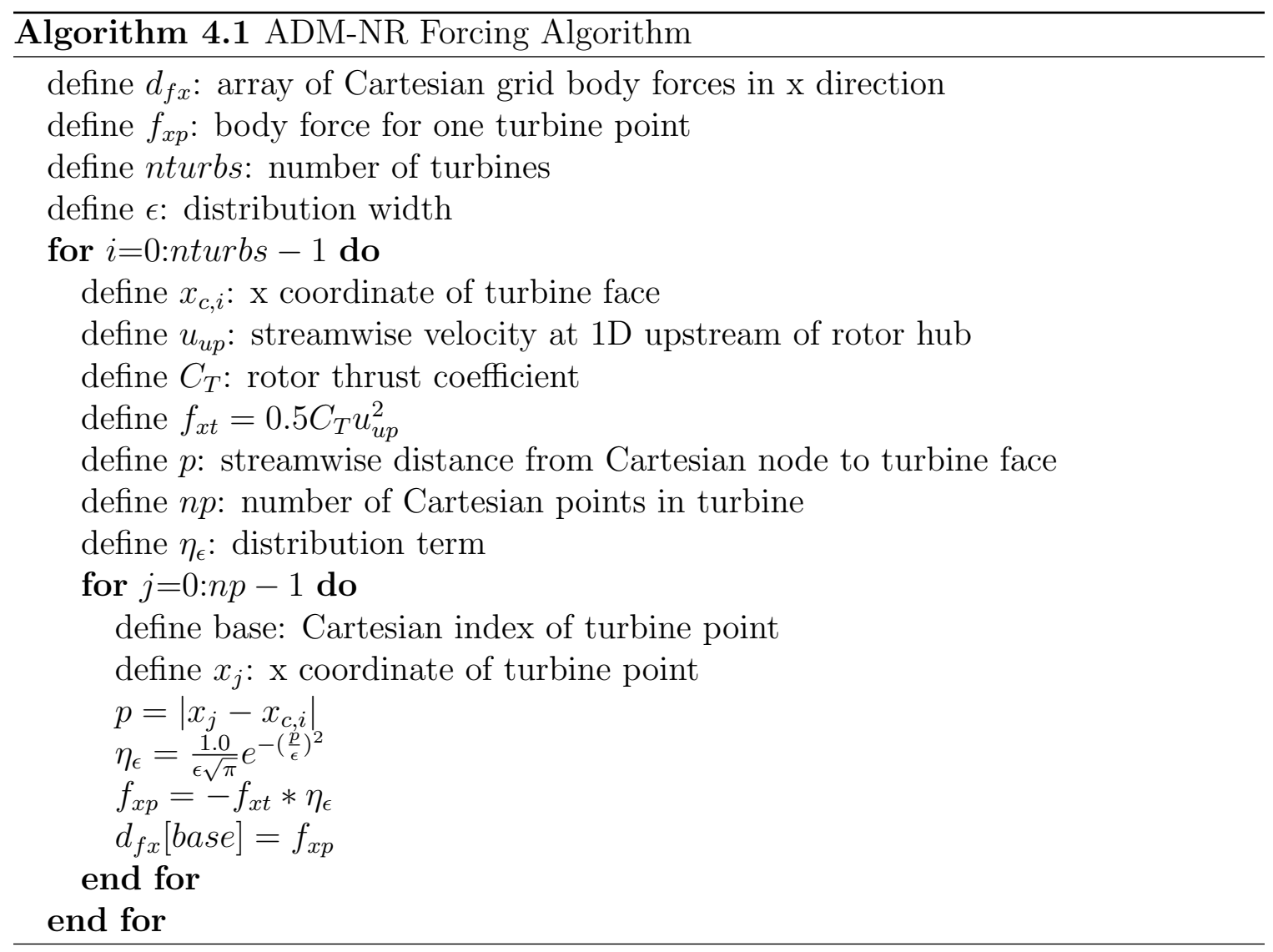

When considering use of the power coefficient, $C_{P}$, it is worth noting that the overall turbine efficiency is

$$
\eta_{o}=\eta_{m} C_{P}=\frac{P_{\text {out }}}{\frac{1}{2} \rho A U^{3}},
$$

where $\eta_{m}$ is the mechanical and electrical efficiency of the turbine [34]. This means the resulting power from the formulation $P=\frac{1}{2} \rho A C_{P} \bar{U}^{3}$ represents the power produced at the rotor and not the overall power form the turbine.

We propose a first-principle based approach that is scalable to the number of turbines in a wind farm. The turbine wake models will be paired with a control volume analysis approach. This approach can analyze kinetic energy changes in a 


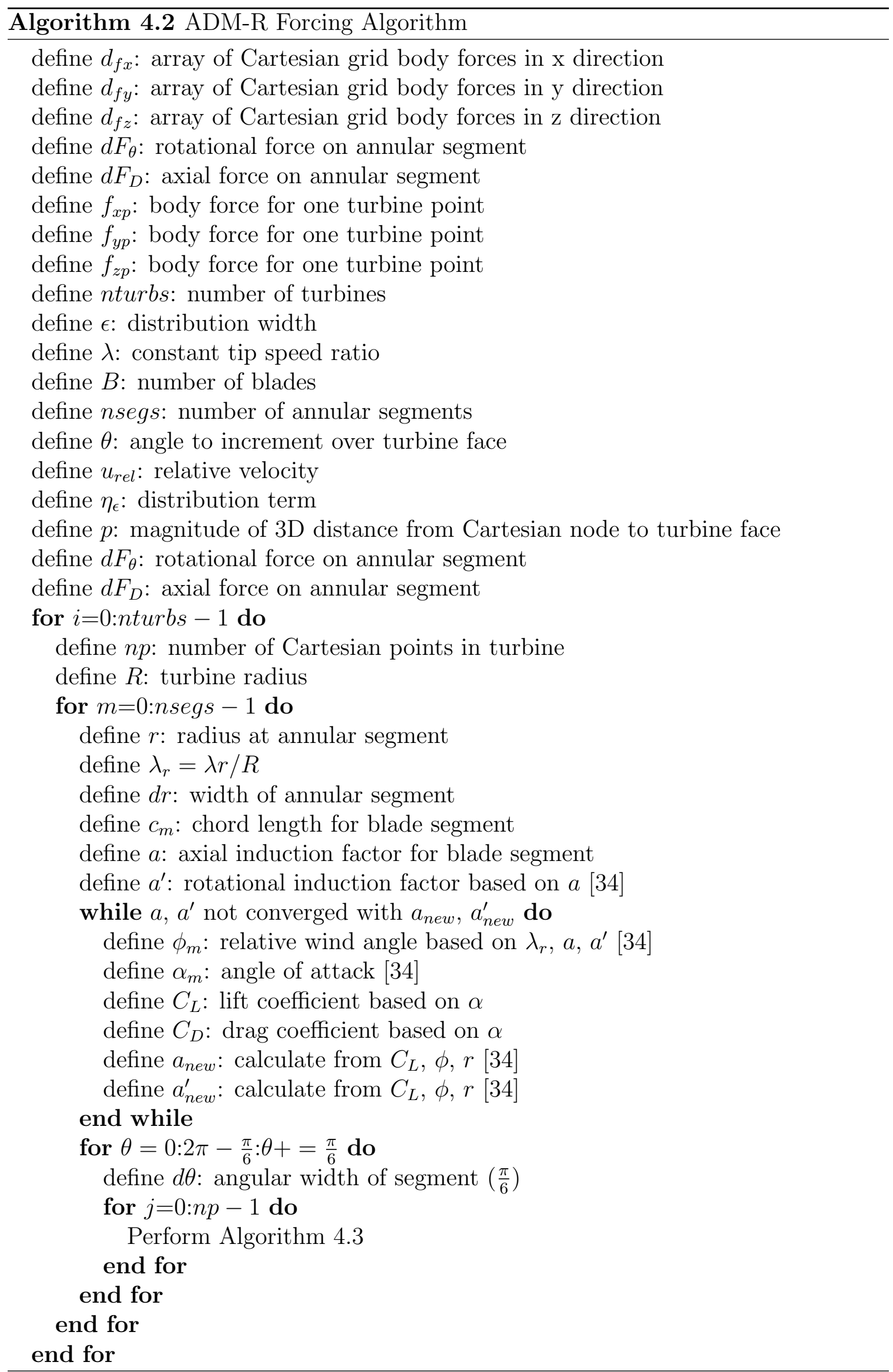




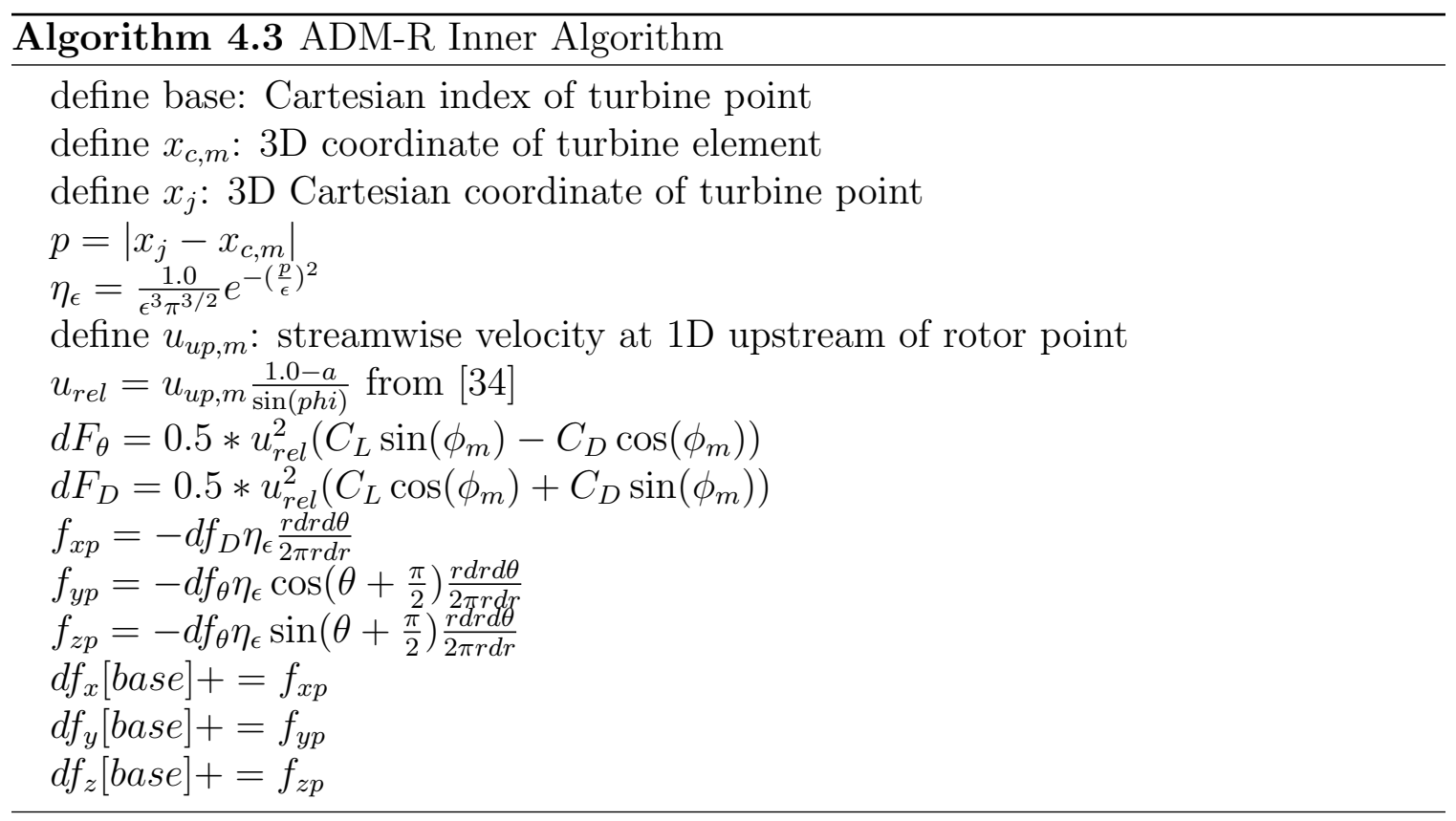

control volume for single and multiple turbine arrangements. Also, it relies less on the assumption of available upstream power in the wind when evaluating power in complex terrain environments. Our assertion is that this will accurately predict the power extracted from the air by turbines and provide an effective tool for wind power forecasting and farm design. This method does not take into account the turbine efficiency. The efficiency of the gearbox and generator will need to be considered in order to reach power produced by the turbine.

The CV method starts with a rectangular box surrounding the wind turbine or farm. To solve for the power removed from the airflow, the conservation total of energy can be solved around the CV using

$$
\frac{d E}{d t}=\dot{Q}-\dot{W}+\sum_{\text {in }} \dot{m}_{\text {in }}\left(h_{\text {in }}+\frac{U_{\text {in }}^{2}}{2}+g z_{\text {in }}\right)-\sum_{\text {out }} \dot{m}_{\text {out }}\left(h_{\text {out }}+\frac{U_{\text {out }}^{2}}{2}+g z_{\text {out }}\right) .
$$

In this equation, $E$ is energy, $\dot{Q}$ is heat flux, $\dot{W}$ is the rate of change of work, 
Table 4.1: Summary of power estimation methods

\begin{tabular}{|c|c|c|c|}
\hline Reference & $\begin{array}{l}\text { Reported Power } \\
\text { Method }\end{array}$ & Pros & Cons \\
\hline $\begin{array}{l}\text { Porté-Agel et al. } \\
{[50]}\end{array}$ & $\begin{array}{l}\text { Drop in upstream } \\
\text { power } P=\frac{1}{2} \rho A \bar{U}^{3} \text {. } \\
\bar{U} \text { is average velocity } \\
\text { over the rotor face at } \\
1 \text { rotor diameter (D) } \\
\text { upwind. }\end{array}$ & $\begin{array}{l}\text { Straightforward } \\
\text { computation. }\end{array}$ & $\begin{array}{l}\text { Assume no change in } \\
\text { percent of power ex- } \\
\text { tracted from wind. }\end{array}$ \\
\hline $\begin{array}{lr}\text { Wu } & \text { and } \\
\text { Porté-Agel } & {[72],} \\
\text { Martínez-Tossas } \\
\text { et al. } \quad[36], \\
\text { Martinez } \\
\text { al. }[35]\end{array}$ & $\begin{array}{l}\text { Product of rotor speed } \\
\text { and torque. Torque is } \\
\text { the rotational force in- } \\
\text { tegrated over the ro- } \\
\text { tor. }\end{array}$ & $\begin{array}{l}\text { Provides power } \\
\text { from rotor me- } \\
\text { chanics. }\end{array}$ & $\begin{array}{l}\text { Requires knowledge of } \\
\text { rotor speed at vary- } \\
\text { ing velocities and con- } \\
\text { ditions. }\end{array}$ \\
\hline $\begin{array}{l}\text { Stevens and } \\
\text { Meneveau [59] }\end{array}$ & $\begin{array}{l}\text { Power curve } P= \\
\frac{1}{2} \rho C_{P} A \bar{U}^{3} \cdot \bar{U} \text { is aver- } \\
\text { age velocity over the } \\
\text { rotor face at } 1 \text { rotor } \\
\text { diameter }(D) \text { upwind. }\end{array}$ & $\begin{array}{l}\text { Easy for- } \\
\text { mulation } \\
\text { performs well } \\
\text { in predictable } \\
\text { terrain. }\end{array}$ & $\begin{array}{l}\text { Power curve varies } \\
\text { with turbine and } \\
\text { environment. }\end{array}$ \\
\hline Current study & $\begin{array}{l}\text { Control volume en- } \\
\text { ergy budget }\end{array}$ & $\begin{array}{l}\text { Scalable and } \\
\text { first-principle } \\
\text { based. }\end{array}$ & $\begin{array}{l}\text { Unverified in this ap- } \\
\text { plication. Need tur- } \\
\text { bine efficiency as in- } \\
\text { put. }\end{array}$ \\
\hline
\end{tabular}

$h$ is enthalpy, and $g$ is acceleration due to gravity. It is assumed that enthalpy change, elevation change, and viscous losses can be neglected and that density remains constant. As such, the equation simplifies to

$$
\dot{W}=\rho \int_{S}\left(\frac{u_{i} u_{i}}{2} u_{j} n_{j}\right) d S
$$

Here, $\dot{W}$ is the power extracted by the turbines(s). The CV analysis for the extracted power simplifies to 


$$
\dot{W}=\sum_{\text {in }} \dot{m}_{\text {in }} \frac{U_{\text {in }}^{2}}{2}-\sum_{\text {out }} \dot{m}_{\text {out }} \frac{U_{\text {out }}^{2}}{2}
$$

Numerically, this is simple to calculate over the 6 planes making up the CV in the code. Furthermore, this form makes tracking the change in extracted power with time a straightforward operation. 


\section{CHAPTER 5}

\section{RESULTS}

This chapter outlines the results of simulations to validate the immersed boundary method for complex terrain flows and turbine wake modeling. It includes comparison of the wake models simulations against well documented cases, and application of the wake models to simulate turbine wakes in complex terrain.

\subsection{Wind Flow over Complex Terrain}

Two benchmark cases were chosen to validate the flow over complex terrain. The cases were Bolund Hill [6, 7] in Denmark and Askervein Hill [63, 64] in Scotland. Bolund Hill was chosen because of the detailed field study as well as the vertical profiles from the 1:115 scale wind tunnel simulation [76]. While the field study collected a large amount of data and provided a well documented real world look at flow over complex terrain, the wind tunnel was a controlled, repeatable experiment. The simulation parameters for both Bolund Hill and Askervein Hill are summarized in Table 5.1 and Table 5.2. Both the wind tunnel and full scale cases of Bolund Hill were simulated using GIN3D to explore the sensitivity and ability to match the complex flow features. 
Table 5.1: Summary of simulation parameters for Field Study (FS) and Wind Tunnel (WT) cases.

\begin{tabular}{|c|c|c|c|}
\hline Case & Domain Size $\mathbf{( m )}$ & $z_{0} \mathbf{( m )}$ & Periodic Shift $\mathbf{( m )}$ \\
Askervein FS & $8218 \times 5800 \times 1000$ & 0.03 & 1000 \\
Bolund FS & $1533 \times 765 \times 127$ & $6.0 \times 10^{-4}$ & 150 \\
Bolund WT & $10.5 \times 2.2 \times 2.2$ & $1.02 \times 10^{-5}$ & 0.4 \\
\hline
\end{tabular}

Table 5.2: Summary of mesh sizes for Field Study (FS) and Wind Tunnel (WT) cases.

\begin{tabular}{|c|c|c|c|}
\hline Case & Mesh 1 & Mesh 2 & Mesh 3 \\
Askervein FS & $321 \times 257 \times 129$ & $513 \times 385 \times 193$ & $641 \times 513 \times 257$ \\
Bolund FS & $385 \times 193 \times 129$ & $513 \times 257 \times 193$ & $769 \times 385 \times 257$ \\
Bolund WT & N/A & N $/ \mathrm{A}$ & $1025 \times 193 \times 513$ \\
\hline
\end{tabular}

\subsubsection{Bolund Hill Full Scale}

Bolund Hill is an approximately $12 \mathrm{~m}$ tall hill located directly off the coast of Denmark. During certain times of the year it is completely surrounded by water. Rather than looking at the flow along the hill as in Figure 2.4, the vertical profiles at four masts were compared. This was done to give a detailed look at the flow as it changes in the vertical direction rather than at one height above the ground. Figure 5.1 shows the topography of Bolund Hill as well as the mast locations and wind direction for line $\mathrm{B}$ with the $270^{\circ}$ wind direction as described in [7].

For the full scale simulation we used a domain size of $1533.0 \mathrm{~m} \times 765.0 \mathrm{~m} \times 127.0$ $\mathrm{m}$ and investigated the senitivity to mesh size with the meshes detailed in Table 5.2. We use the blending height $h_{B}=5.3 \mathrm{~m}$ which satisfies the relationship $h_{B}(2 \Delta)^{-1}$ for the three meshes as suggested in [55] to make certain that the LES scheme is not applied in areas without proper resolution. For these simulations, the top boundary condition was set to a zero-shear-stress wall, and the lateral walls are periodic. The inlet and outlet are set to shifted periodic [44]. The shifted periodic is implemented in order to avoid the locking of flow structures in the spanwise direction. This is 


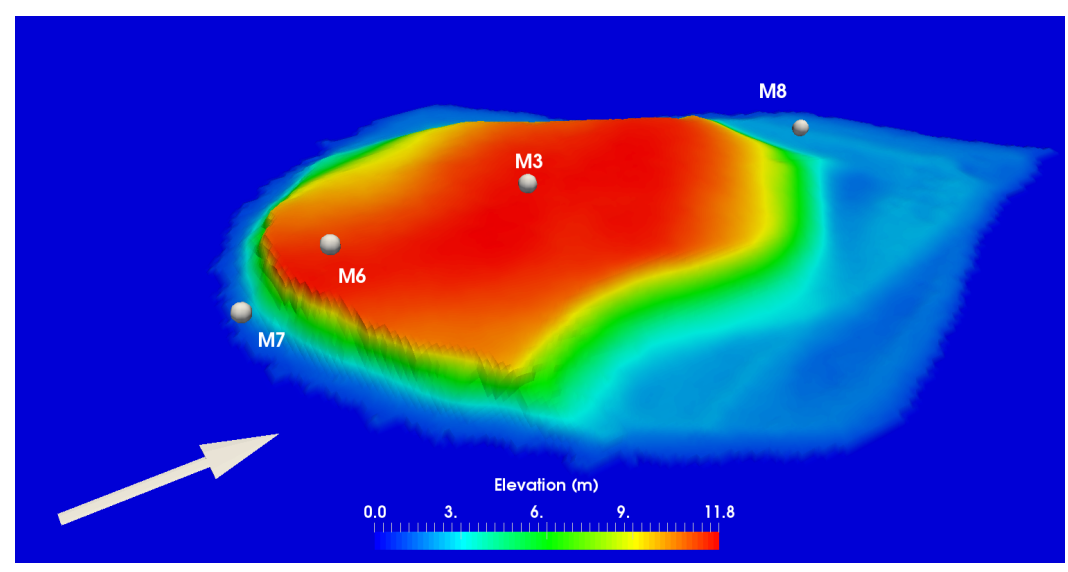

Figure 5.1: Elevation map of Bolund Hill with mast locations and wind direction.

done by shifting the outflow a specified distance in the spanwise direction during the periodic recycling. This helps decorrelate the flow structures near the boundary, allowing for shortened domains over traditional periodic configuration. The periodic shift for each simulation is detailed in Table 5.1. The aerodynamic roughness length was set to $0.0006 \mathrm{~m}$.

We calculated the fractional speedup along line B at M7, M6, M3, and M8 from the field study. The fractional speedup, $S$, is calculated by

$$
S=\frac{U\left(h_{a g l}\right)-U_{0}\left(h_{a g l}\right)}{U_{0}\left(h_{a g l}\right)},
$$

where $U\left(h_{a g l}\right)$ is wind speed $U=\sqrt{U_{x}^{2}+U_{y}^{2}}$ at height above ground level and $U_{0}$ is wind speed at height above ground level at the reference location. Figure 5.2 shows the results of the full scale simulation. The results show minor sensitivity to mesh size. The largest difference is seen at M6, which is only slightly downwind of the escarpment and in a region of flow separation. An attached flow assumption is used for the formulation of the IB method and the SGS model which affects our accuracy in the flow separation regions. All three meshes show good agreement in the attached 
flow regions at M7 and M3.

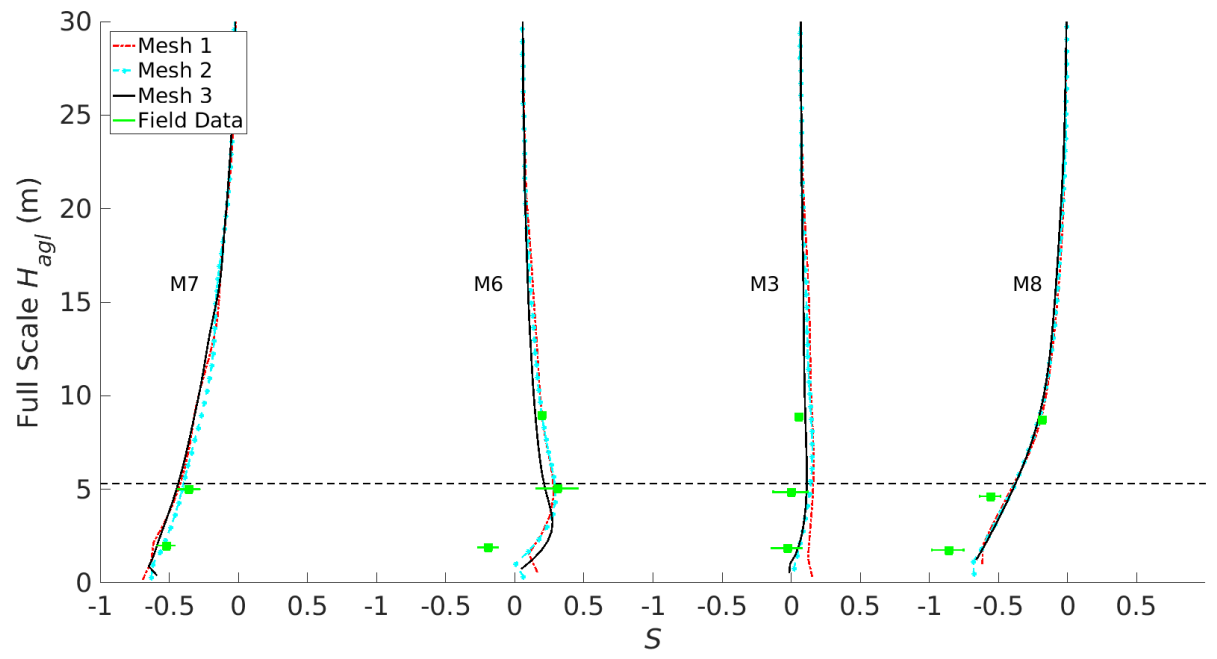

Figure 5.2: Fractional speedup for full scale mesh study along line B with $270^{\circ}$ wind direction compared with full scale field data [6] vertical profiles at masts $7,6,3$, and 8. Mesh 1 is the most coarse.

\subsubsection{Bolund Hill Wind Tunnel}

Yeow et al. performed a detailed wind tunnel experiment of Bolund Hill using a 1:115 scale model and collecting vertical velocity profiles using particle image velocimetry (PIV) [76]. We used a computational domain of $10.5 \mathrm{~m} \times 2.2 \mathrm{~m} \times$ $2.2 \mathrm{~m}$ to match the size of the wind tunnel. The simulation was performed with one mesh size of $1025 \times 193 \times 513$ in the streamwise, spanwise, and vertical directions. This corresponds to a full scale mesh spacing of $1.2 \mathrm{~m} \times 1.2 \mathrm{~m} \times 0.5 \mathrm{~m}$. Following the wind tunnel experiment, the Reynolds number was set to $4.15 \times 10^{5}$ based on the hill height and the aerodynamic roughness was set to $z_{0}=1.02 \times 10^{-5}$. The lateral boundary conditions are the same as the full scale Bolund Hill case. The flow rate was set to $38.39 \mathrm{~kg} \mathrm{~s}^{-1}$ and the periodic shift was set to $0.4 \mathrm{~m}$. The RANS-LES blending 
height was set to $0.0217 \mathrm{~m}$, corresponding to a full scale value of $2.5 \mathrm{~m}$. To better match the conditions of the wind tunnel, we modeled the top wall as a smooth-surface, wall-shear stress boundary conditions using the Schumann-Grötzbach model $[26,54]$ with standard smooth-wall log-law values with von Kármán constant set to 0.41 and log-law intercept constant of $5.2[49]$.

Figure 5.3 compares the GIN3D simulation fractional speed-up to the wind tunnel experiment vertical profiles and the full scale field data as well as the water tunnel experiment from the Bolund Experiment [6]. The reference location is consistent with the wind tunnel experiment [76]. There is good agreement with the three benchmark cases for M7. There is disagreement between all three benchmarks near the surface on M6, but the IB method falls in between the field study and the wind tunnel results. Yeow et al. attributes the disagreement to intermittent recirculation downwind of the escarpment near M6 and a difference in the ratio of boundary layer height to hill height than is observed for the full scale case [76]. There is general agreement for all 4 masts further away from the surface.

\subsubsection{Askervein Hill}

The Askervein hill project was a study of flow over a 116m high hill on a Scottish island [64]. The study looked at three different profiles over the hill with 10 m masts. Figure 5.4 shows the wind field around Askervein Hill along with two profiles over the hill. For these simulations, the boundary conditions are the same as in the full scale Bolund Hill case. The periodic shift is set to $1000 \mathrm{~m}$.

The first set of simulations performed studied the effect of the turbulence model. Figure 5.5 and Figure 5.6 show the comparison of GIN3D with the field study [63] of Askervein along the A and AA lines. We tested the RANS-LES blending height at 4 


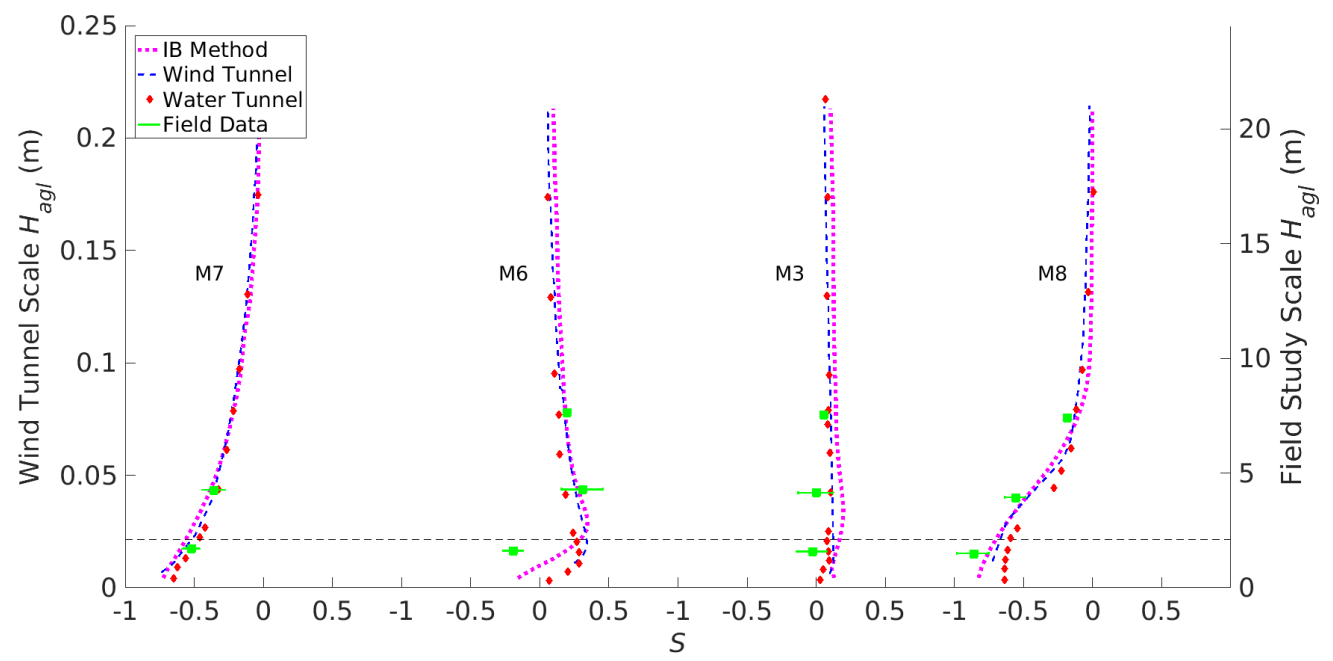

Figure 5.3: Fractional speedup of scale simulation along line B with $270^{\circ}$ wind direction compared with wind tunnel [76], water tunnel, and full scale [6] vertical profiles at masts $7,6,3$, and 8 .

heights. There is good agreement on the windward side of the hill for both profiles, with little sensitivity to the blending height. This is consistent with the attached flow assumptions present in the formulation of the IB method used here. On the leeward side of the hill there is some flow separation. There is some deviation between the simulation and field data in this region. There is also considerable sensitivity to the blending height in this region. By looking at Figure 5.4, it is evident that the $\mathrm{A}$ line is in a region of larger flow separation This is due to the fact that it passes behind a taller portion of the hill. The attached flow assumption explains the larger sensitivity present in the region of flow separation, but we note that this does not prevent us from capturing the separation. Based on these results, we chose to perform our mesh sensitivity study using the $35 \mathrm{~m}$ blending height, which has the best agreement with the field data.

To explore the sensitivity of the IB method to mesh resolution, we used the grids 


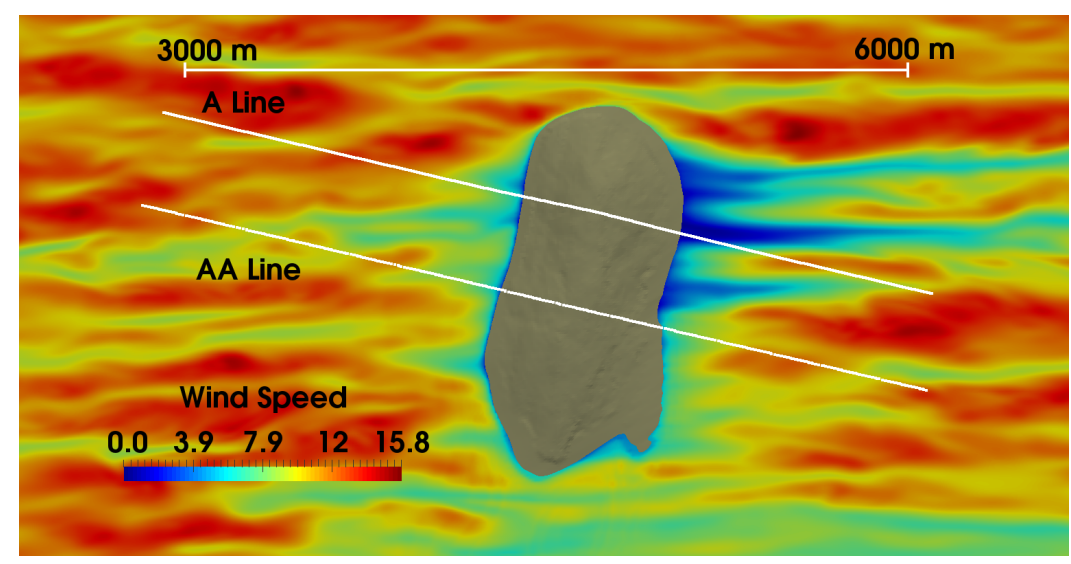

Figure 5.4: Visualization of velocity field around Askervein Hill showing the measurement profiles.

detailed in Table 5.2. We use the blending height $h_{B}=35 \mathrm{~m}$ which satisfies the relationship $h_{B}(2 \Delta)^{-1}$ for the three meshes as suggested in [55]. The fractional speedup along the A and AA line similar to the blending height study are shown in Figure 5.7 and Figure 5.8.

There is good agreement on the windward side of the hill for both the A and AA line. There is relatively good agreement along the entire AA line with some mesh sensitivity. There is a significant drop in speedup in the A line on the windward side for the coarsest mesh. This can be attributed to the lack of mesh resolution (25 $\mathrm{m}$ spacing in the streamwise direction). This mesh is unable to capture a slight convexity on the surface that is present at a point along the A line. There is low mesh sensitivity along the A line at the hill top and for the first portion of the leeward side. Similar to the blending height study, there is more sensitivity further downstream along the $\mathrm{A}$ line. An adaptive mesh resolution supporting finer mesh resolutions would be necessary to capture the small terrain features present further downstream along the A line. These results show that the IB method works well on various methods as long as the terrain is sufficiently resolved. 


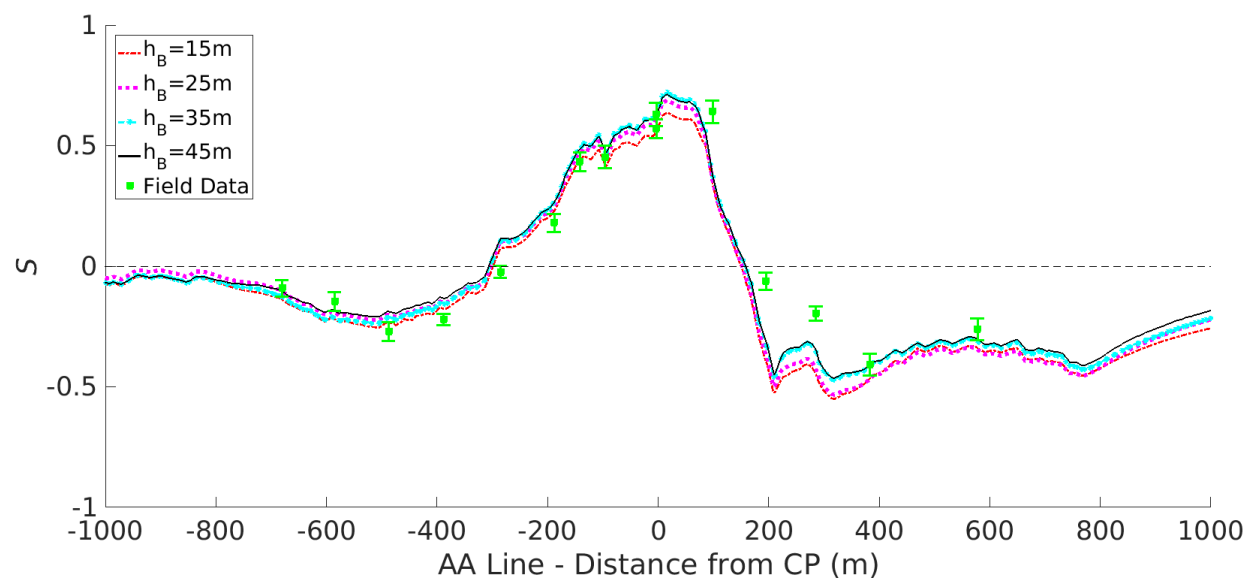

Figure 5.5: Fractional speed up along Askervein AA Line showing sensitivity to RANS-LES blending height, $h_{B}$. The field data is from [69].

\subsection{Wake Models}

Figure 5.9 displays the results of the ADM-NR and ADM-R simulation of the wind tunnel experiment [12] and the corresponding ADM simulations [71]. To simulate the wind tunnel, a domain of $6.5 \mathrm{~m} \times 0.72 \mathrm{~m} \times 0.5 \mathrm{~m}$ is used with $385 \times 129 \times 129$ points. The aerodynamic roughness is $0.03 \mathrm{~mm}$ and the friction velocity $\left(u^{*}\right)$ is 0.102 $\mathrm{m} / \mathrm{s}$. The simulations are run for a total time of 40 seconds. The boundary conditions are the same as the full scale Bolund Hill case with a periodic shift of $0.115 \mathrm{~m}$.

The thrust coefficient for the turbine is 0.529 , corresponding to the cited power coefficient [71] and the distance width for the Gaussian distribution function is set to 2 times the stream-wise grid spacing. The nacelle is forced in the same manner as [71] and the tower is neglected. For the rotational model, the necessary parameters for the blade are taken from [71] and the distance width is set equal to the stream-wise grid spacing.

The ADM-NR almost exactly matches the comparable ADM-NR simulation and shows the expected under-prediction in velocity drop in the near wake and the good 


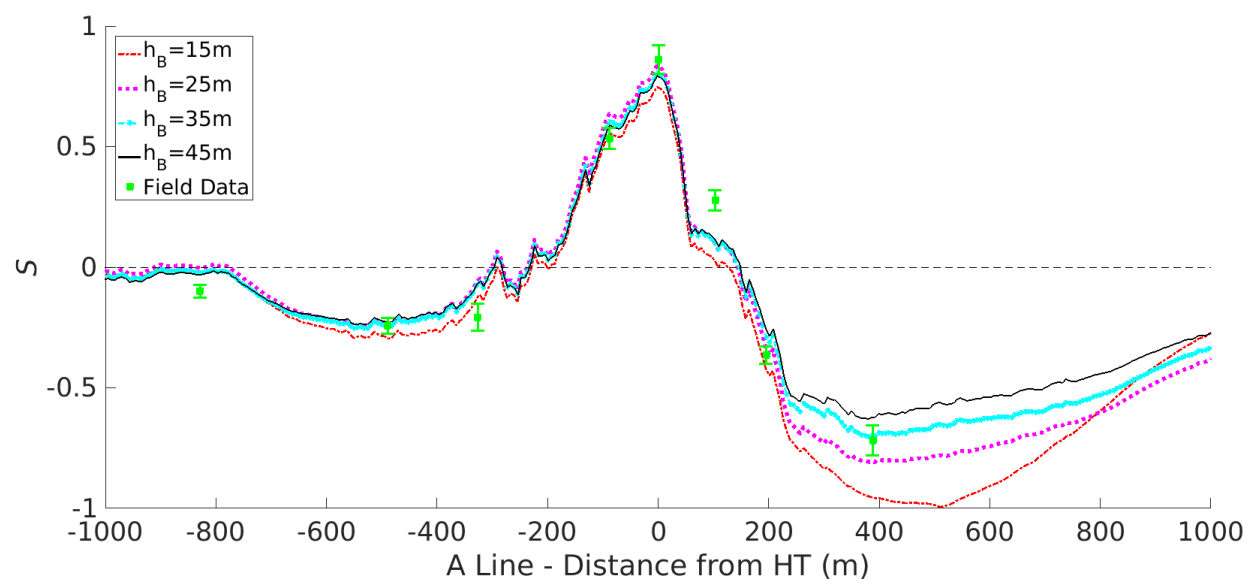

Figure 5.6: Fractional speed up along Askervein A Line showing sensitivity to RANSLES blending height, $h_{B}$. The field data is from [69].

agreement with the experimental data and corresponding ADM-R results in the far wake.

For the ADM-R simulation, $C_{L}$ and $C_{D}$ were found in an iterative manner [34] to match the approach in the corresponding simulations [71]. The lift and drag profiles were obtained from the equations in Table 1 of Sunada et al. for the flat plate airfoil 5 [61] similarly to [71]. The results show very good agreement of the rotational model with both the experimental results and the results from the corresponding simulations.

In order to validate the methods in the full scale, an operational wind farm was simulated. Figure 5.10 shows the layout of 5 turbines (Siemens SWT-2.3-93) that make up a section of an operational wind farm located in Mower County, Minnesota [50]. The field study of the wind farm involved collection of velocity measurements from two sonic detection and ranging (SODAR) stations that took measurements approximately every 10 seconds from a 10 minute period. Porté-Agel et al. simulated the wind farm for a period of 1.5 hours in order to guarantee quasi-steady flow. The results are for the last half hour of the simulated time [50]. 


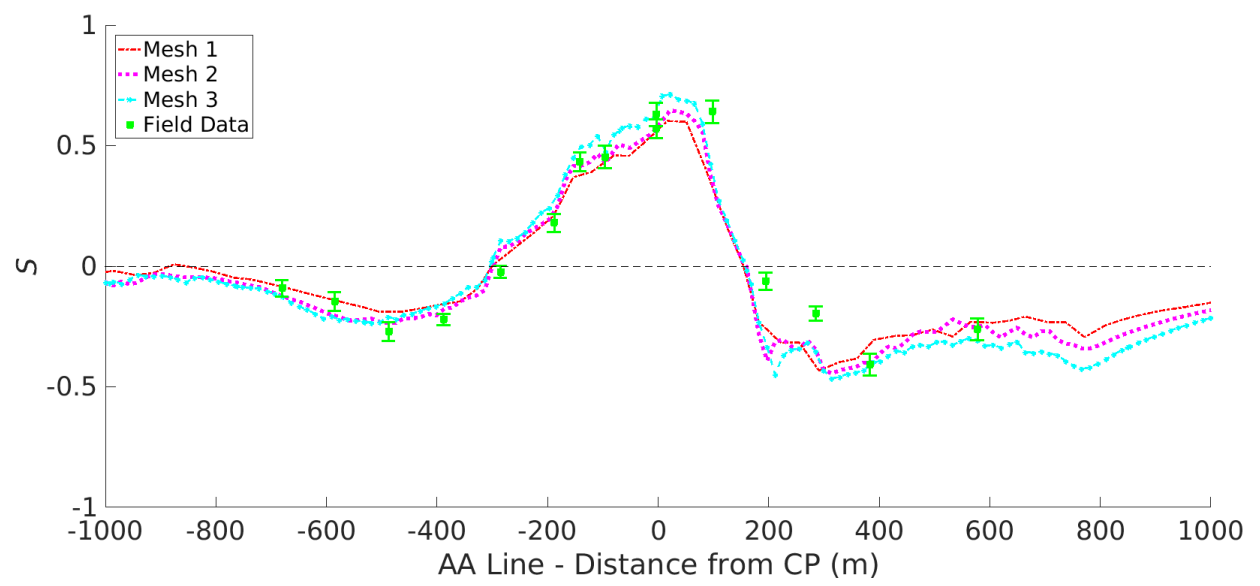

Figure 5.7: Fractional speed up along Askervein AA Line showing sensitivity to mesh resolutions in Table 5.2. The field data is from [69]

The computational domain for the GIN3D simulations measures $4000 \mathrm{~m} \times 1200$ $\mathrm{m} \times 725 \mathrm{~m}$ with a grid size $513 \times 257 \times 129$ points. The simulations are run for 3 hours of physical time with the same boundary conditions as the full scale Bolund Hill case. The periodic shift is set to $400 \mathrm{~m}$. The thrust coefficient is set to 0.57 [50] for the ADM-NR simulations and the distance width is set to equal to the stream wise grid spacing for the ADM-NR and ADM-R. The lift and drag coefficients are found from the $C_{L}$ and $C_{D}$ curves in Laursen et al. [31], pictured in Figure 5.11, similarly to [50]. As $C_{L}$ and $C_{D}$ are reported as a function of radius, they do not need to be iteratively solved in this specific case. The chord length as a function of radius is assumed to be the same as in [14]. The aerodynamic roughness is set equal to $0.1 \mathrm{~m}$.

Figure 5.12 shows the results from a simulation the wind farm [50]. S1 indicates the wind profile from the SODAR station in the free stream and S2 indicates the wind profile from the SODAR station that is installed approximately 4.25 turbine diameters downstream from one of the turbines. The GIN3D results show good agreement with field data as well as the numerical results from Porté-Agel et al. [50]. 


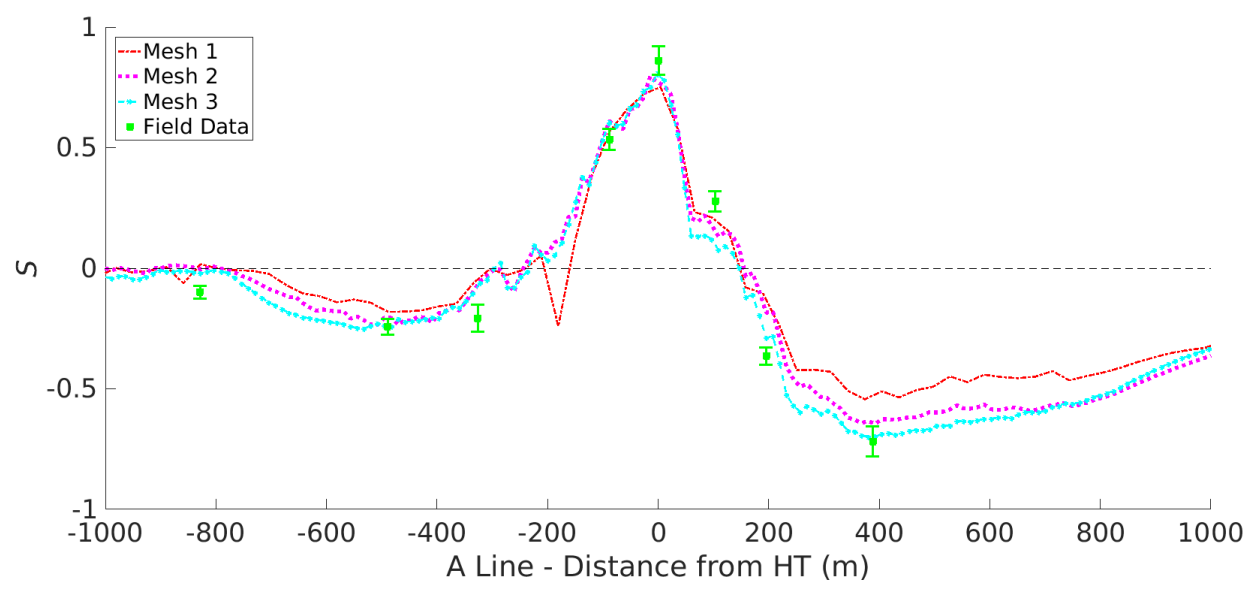

Figure 5.8: Fractional speed up along Askervein A Line showing sensitivity to mesh resolution in Table 5.2. The field data is from [69]

There is a slight mismatch near the surface for the vertical velocity profile at S1. This can be attributed to use the periodic inlet and outlet, which is not able to fully capture the inlet profile created by the periodic buffer zone technique used in Porté-Agel et al. [50]. At S2, the ADM-NR simulation shows good agreement with the corresponding simulation and the expected under-prediction in velocity drop. The ADM-R shows agreement with the field data, the corresponding ADM-R simulation, and the ALM simulation [50].

\subsection{Control Volume Analysis for Power Estimation}

The control volume energy budget was used to investigate the power drop between T41 and T42 from the Mower County wind farm pictured in Figure 5.10. Porté-Agel et al. reported that the supervisory control and data acquisition (SCADA) data from the wind farm showed a $48 \%$ reduction in power [50]. The authors calculated available upstream power at one diameter upstream of the rotors as $P=0.5 \rho A \bar{U}_{\text {rotor }}^{3}$. They found a $37 \%$ reduction for the ADM-NR, a $47 \%$ reduction for the ADM-R, 

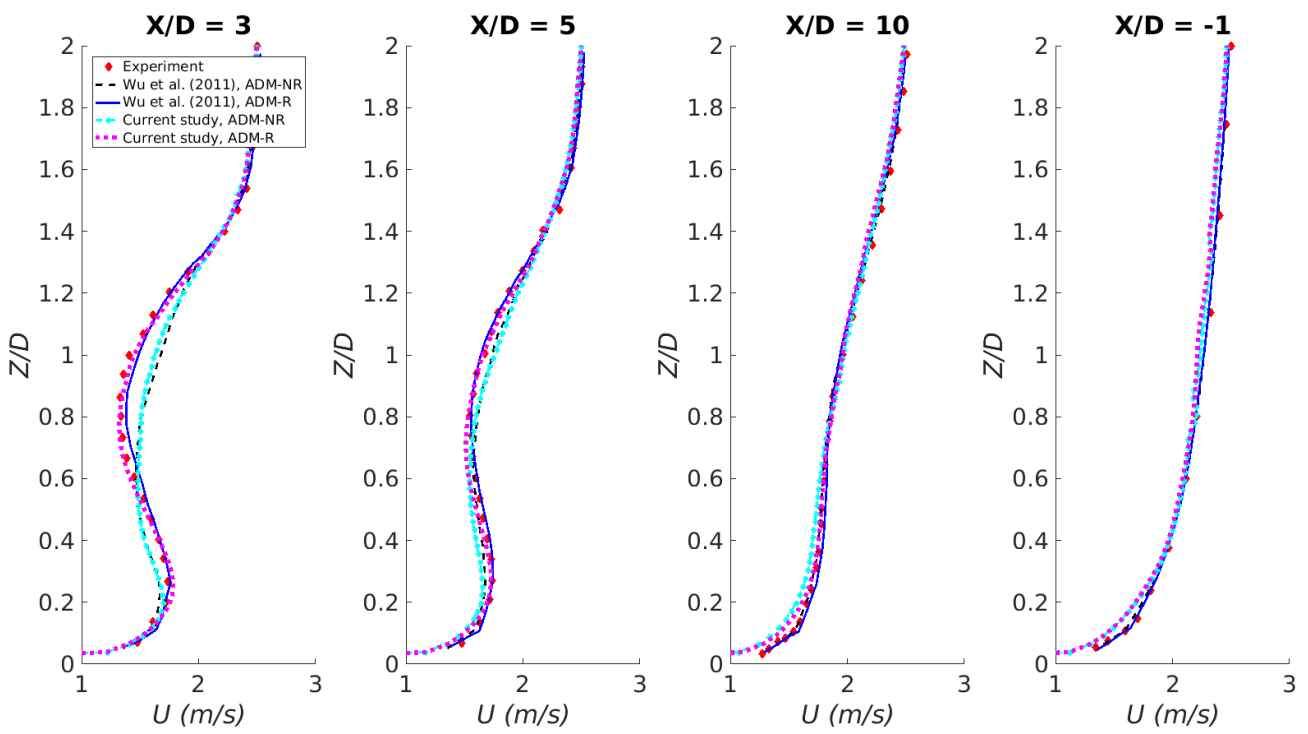

Figure 5.9: Streamwise velocity profiles wind turbine wind tunnel experiment and simulations. $Z / D$ refers to height above ground level normalized by the turbine diameter. Results are shown from the wind tunnel experiment from Chamorro and Porté-Agel [12], ADM-NR and ADM-R simulations [71], and the ADM-NR and ADM$\mathrm{R}$ results from the current study using GIN3D. The velocity profiles are at 3D (3 diameters), 5D, and 10D downstream as well as 1D upstream.

and a $50 \%$ reduction for the ALM. The percent power reduction was calculated using $\left(r_{P}=\left(1-P_{T 42} / P_{T 41}\right) \times 100 \%\right)[50]$.

In order to investigate the power extracted from the wind, we used the same computational domain as outlined in the previous section, but used a turbulent perturbation inflow method $[17,67]$ to avoid any correlation resulting from the periodic inflow and outflow previously used and to decrease the flow development times associated with periodic simulations. We use a friction velocity of $0.68 \mathrm{~m} / \mathrm{s}$ and a $z_{0}$ of $0.3 \mathrm{~m}$. The inlet profile is depicted in Figure 5.13. Using the same power estimation methods as Porté-Agel et al. [50] we investigated the drop in upstream power and then applied our proposed control volume analysis to estimate power extraction from the wind. The results are shown in Table 5.3. Both the rotational 


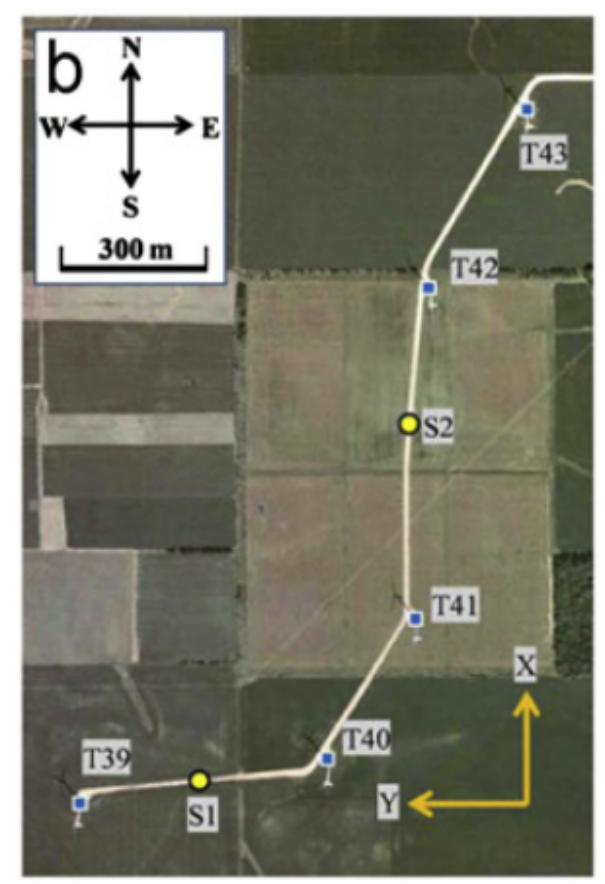

Figure 5.10: Wind turbine and SODAR station locations for the subset of the wind farm in Mower County. S1 and S2 represent SODAR stations and T39-T43 represent turbines. Image is adopted from [50].

and non-rotational methods show good agreement within the simulation. There is over prediction between the current study and the previous simulations performed by Porté-Agel et al. [50]. They use a buffer zone technique to adjust the very-far-wake downwind flow to an undisturbed boundary layer condition when the flow is recycled. The differences in the profile of the undisturbed flow is evident in Figure 5.13. This could account for differences in reported power drop to the calculated upstream power drop in our simulations.

To test the sensitivity to using available upstream power and the power from manufacturer power curves, two synthetic cases are investigated where two turbines are purposely offset. This allows us to investigate the effects of streamwise turbine spacing. The power curve from Creech et al. [14] is used. The same domain and 


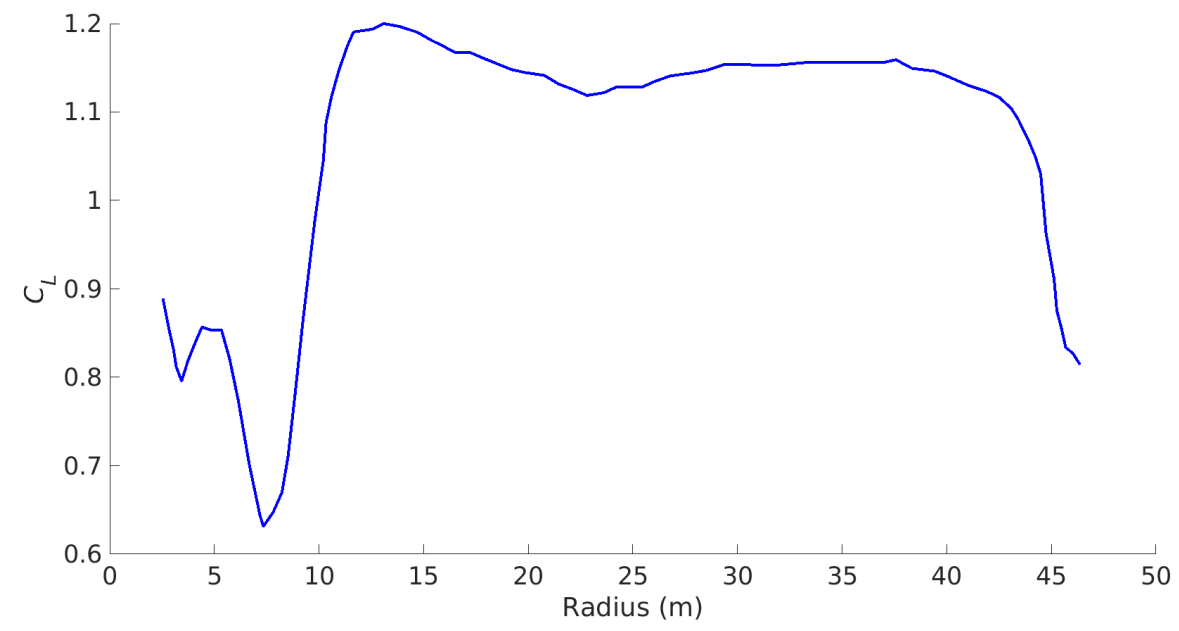

Figure 5.11: $C_{L}$ curve as a function of radius as reported from the $3 \mathrm{D}$ simulations in Laursen et al. [31]. Data is from [31].

Table 5.3: Summary of percent power drop from T41 to T42 in the Mower County wind farm simulation

\begin{tabular}{|l|l|l|l|l|}
\hline Power Method & $\begin{array}{l}\text { Turbine } \\
\text { Model }\end{array}$ & $\begin{array}{l}\text { Porté-Agel et } \\
\text { al. [50] \% drop }\end{array}$ & $\begin{array}{l}\text { GIN3D } \\
\text { \% drop }\end{array}$ & SCADA \\
\hline Upstream & ADM-NR & 37.0 & 41.0 & 48.0 \\
Upstream & ADM-R & 47.0 & 53.9 & 48.0 \\
\hline CV (current study) & ADM-NR & N/A & 44.3 & 48.0 \\
CV (current study) & ADM-R & N/A & 52.2 & 48.0 \\
\hline
\end{tabular}

turbine parameters as the Mower County wind farm are used along with the ADM-R model. We used a friction velocity of $0.665 \mathrm{~m} / \mathrm{s}$ and a $z_{0}$ of $0.3 \mathrm{~m}$.

Case 1 is performed with the turbines spaced approximately $4.3 \mathrm{D}$ apart in the streamwise direction. Case two is tested the turbines spaced approximately 7.3D apart, which is the same spacing T41 and T42 in the Mower County wind farm [50]. In both cases, the turbines are centered in the domain in the spanwise direction and then offset slightly from each-other, following the same spanwise offset as T41 and T42 from the Mower County wind farm. The available upstream power and power resulting from power curve are calculated using velocity sampled at $1 \mathrm{D}, 1.1 \mathrm{D}$, and 
S1

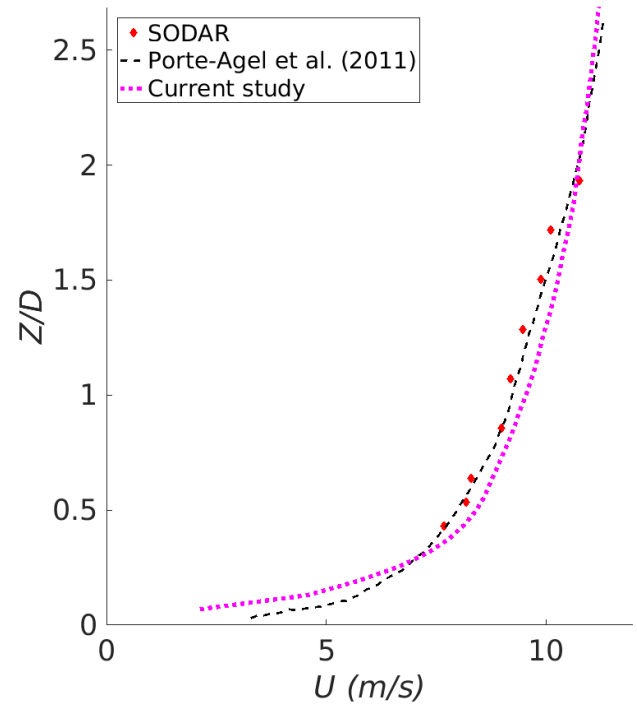

S2

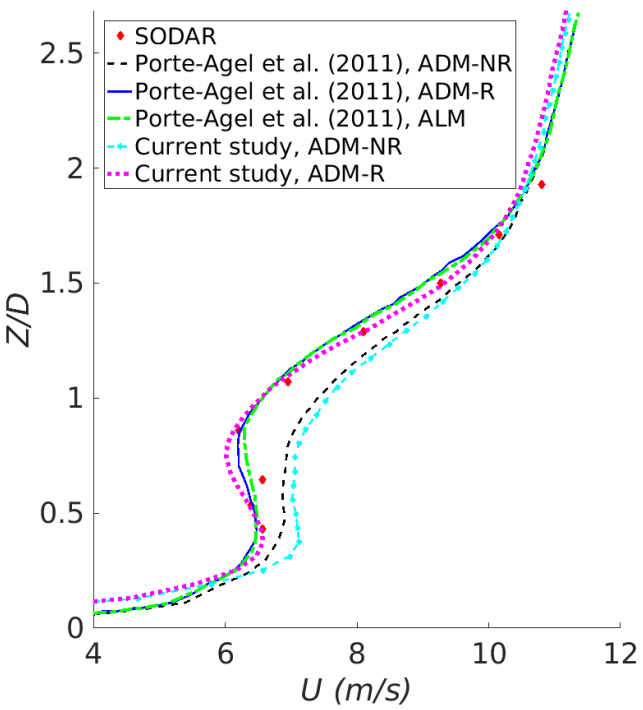

Figure 5.12: Streamwise velocity profile results showing simulation and field data results for the wind farm in Mower County. Results are shown for the SODAR stations, the ADM-NR, ADM-R, and ALM simulations from Porté-Agel [50], and the ADM-NR and ADM-R simulations performed with GIN3D.

1.5D upstream. The velocity is averaged over the rotor area. The power is also calculated using the control volume method. The power drop is summarized in table 5.4 .

There is a small amount of variation in the power drop calculated using these methods. The power curve method predicts a larger power drop from the first to second turbine for both turbine spacings. The CV method shows good agreement with the upstream power drop for the second test case with a turbine spacing of 7.3D. The CV method predicts a lower power drop than either the upstream power method or the power curve method for the test case 1. This is a case with large turbine wake interaction with a smaller turbine spacing than would be recommended for a wind farm. The difference in power drop reported for the current study using the ADM-R in Table 5.3 and the Case 2 current study in Table 5.4 could be a results 


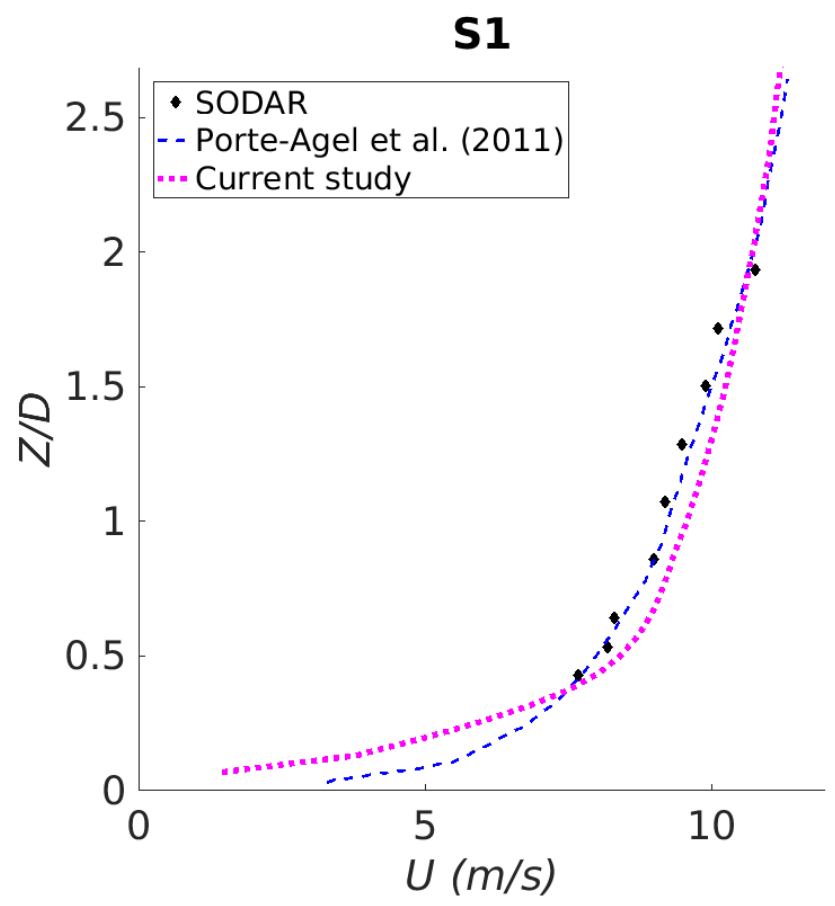

Figure 5.13: Streamwise velocity profiles at SODAR station S1 in the Mower County. Results are shown for the SODAR stations, Porté-Agel et al. simulation [50], the log-law profile, and the current study simulations performed with GIN3D using the turbulent perturbation inflow method.

of the proximity of T41 and T42 to the edge of the domain in the full wind farm simulation, wake effects from T40 in the full wind farm, or the slight difference in friction velocity between the two simulations.

To further investigate the control volume method, we varied the control volume size in the streamwise direction and investigated the power drop using Case 2 from Table 5.4. The results are shown in Table 5.5. The vertical and spanwise sizing of the control volume is large enough to encompass the $93 \mathrm{~m}$ diameter of the rotor. The results show a small sensitivity to the control volume size in the streamwise direction and good agreement with the drop in upstream power and the drop in power using the power curve method. 
Table 5.4: Summary of power losses from test Case 1 (4.3D turbine spacing) and Case 2 (7.3D turbine spacing).

\begin{tabular}{|l|l|l|l|}
\hline Power Method & $\begin{array}{l}\text { Sample } \\
\text { Location }\end{array}$ & $\begin{array}{l}\text { Case 1 Power } \\
\text { Drop (\%) }\end{array}$ & $\begin{array}{l}\text { Case 2 Power } \\
\text { Drop (\%) }\end{array}$ \\
\hline Upstream & 1D & 60.2 & 49.0 \\
& $1.1 \mathrm{D}$ & 60.2 & 48.9 \\
& $1.5 \mathrm{D}$ & 61.4 & 50.4 \\
\hline Power Curve & 1D & 63.1 & 51.1 \\
& $1.1 \mathrm{D}$ & 63.0 & 50.9 \\
& $1.5 \mathrm{D}$ & 64.2 & 52.2 \\
\hline CV (current study) & N/A & 54.8 & 49.2 \\
\hline
\end{tabular}

Table 5.5: Results from the investigation of variation in power drop with control volume size.

\begin{tabular}{|l|l|l|}
\hline Power Method & CV Size $(\mathbf{m})$ & \% Power Drop \\
\hline CV (current study) & $50 \times 100 \times 100$ & 51.9 \\
CV (current study) & $100 \times 100 \times 100$ & 49.2 \\
CV (current study) & $150 \times 100 \times 100$ & 48.7 \\
\hline Upstream & N/A & 49.0 \\
\hline Power Curve & N/A & 51.1 \\
\hline
\end{tabular}

As an application of the current computational capability to large wind farms, we simulate the Horns Rev wind farm off the coast of Denmark. The simulation was run to match the the $270^{\circ}+/-1^{\circ}$ case from Wu et al. [72]. Horns Rev contains 80 Vestas V80 wind turbines, each with a rotor diameter of $80 \mathrm{~m}$ and a hub height of 70 m. The turbines are spaced a minimum of 7 rotor diameters apart in the streamwise and spanwise directions. The blade parameters are the same as the corresponding simulations from $\mathrm{Wu}$ et al. [72]. The ADM-R model is used with a distribution width equal to 2 times the streamwise grid spacing. The $C_{L}$ and $C_{D}$ curves are extracted from the reported curves in [72] as pictured in Figure 5.14.

The normalized power averaged over each row was examined. The computational 


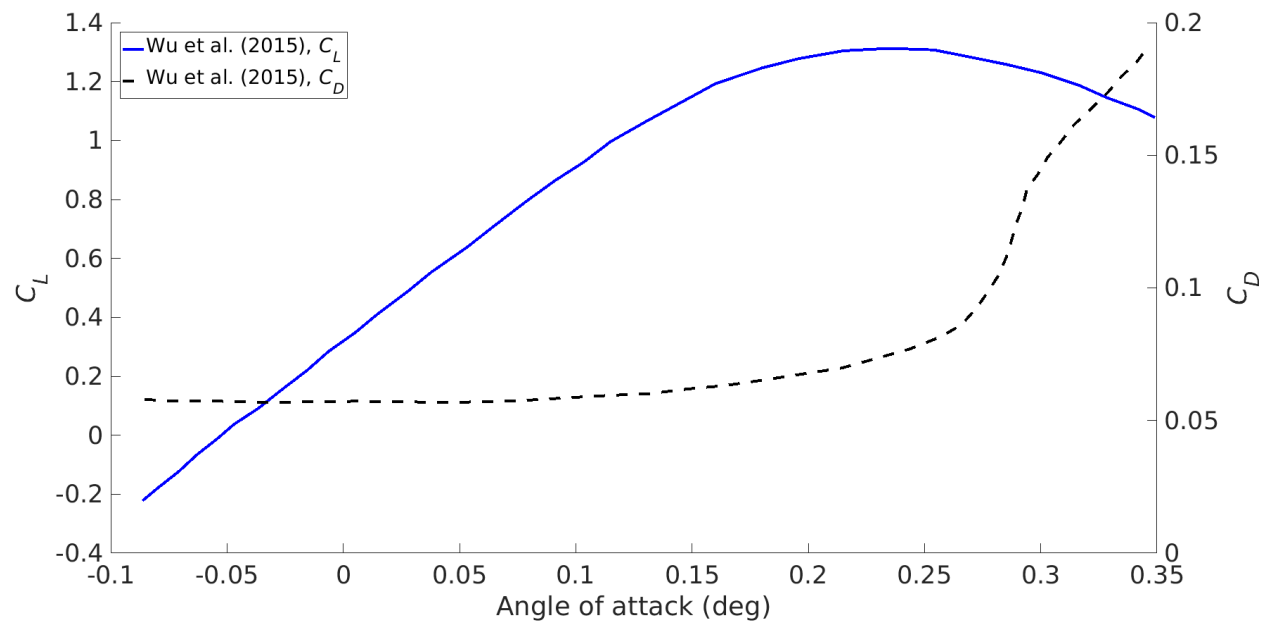

Figure 5.14: $C_{L}$ and $C_{D}$ curves as a function angle of attack as reported in [72]. Data is from [72].

domain measures $23893 \mathrm{~m} \times 9882 \mathrm{~m} \times 1046 \mathrm{~m}$ with a grid size of $1025 \times 641 \times 129$. The aerodynamic roughness length is $0.04 \mathrm{~m}$. The inlet profile is pictured in Figure 5.15. The vertical and spanwise domain sizes are consistent with the corresponding simulation [72] in both length and grid spacing and the streamwise is consistent in grid spacing. The domain is extended beyond the corresponding simulation to allow for synthetic turbulent development using the turbulent perturbation inflow method. The simulations are run for 3 hours and data collection takes place over the last hour. Figures 5.16, 5.17, 5.18, and 5.19 show the simulated flow field around the wind farm. Figures 5.16 and 5.17 show the mean and instantaneous velocity fields at hub height through the farm and Figures 5.18 and 5.19 depict the mean and instantaneous flow around the first column of turbines from the side view.

The ADM-R method was modified for this simulation to dynamically assign the rotational speed. For each turbine, the upstream velocity, $U$, is sampled and used to find the rotational speed, $\Omega$, using the curve depicted in Figure 5.20. These variables 


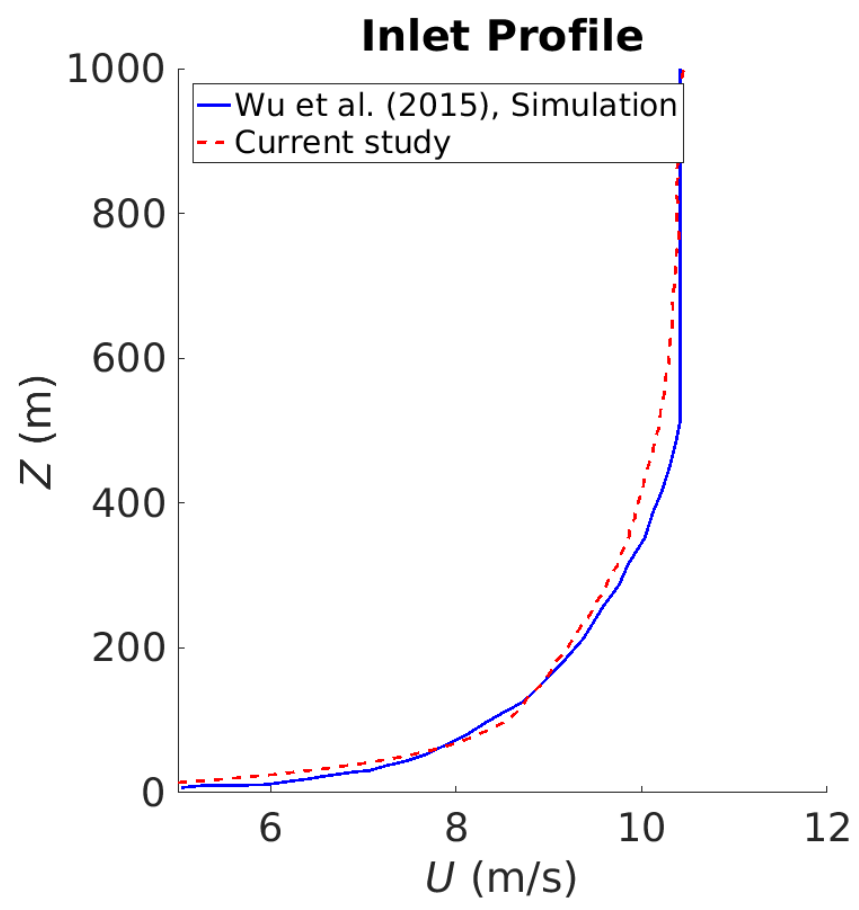

Figure 5.15: Incoming velocity profile for the Horns Rev wind farm showing the current study and the velocity profile in $\mathrm{Wu}$ et al. [72].

are then used to calculate the tip speed ratio,

$$
\lambda=\frac{\Omega R}{U}
$$

where $R$ is the rotor radius. The tip speed is used to calculate the angle of relative wind, $\phi$, as described in section 4.1.2. The power calculated from the current study at each row is normalized by the average power at row one and is shown in Figure 5.21.

Overall, the control volume method works well to predict the normalized power extracted from the wind. The modified method shows improvement over the WindSim [70] software. The model discussed in Wu et al. utilizes a dynamic, iterative rotational speed assignment based on the turbine torque-rotational speed relationship [72]. Their 


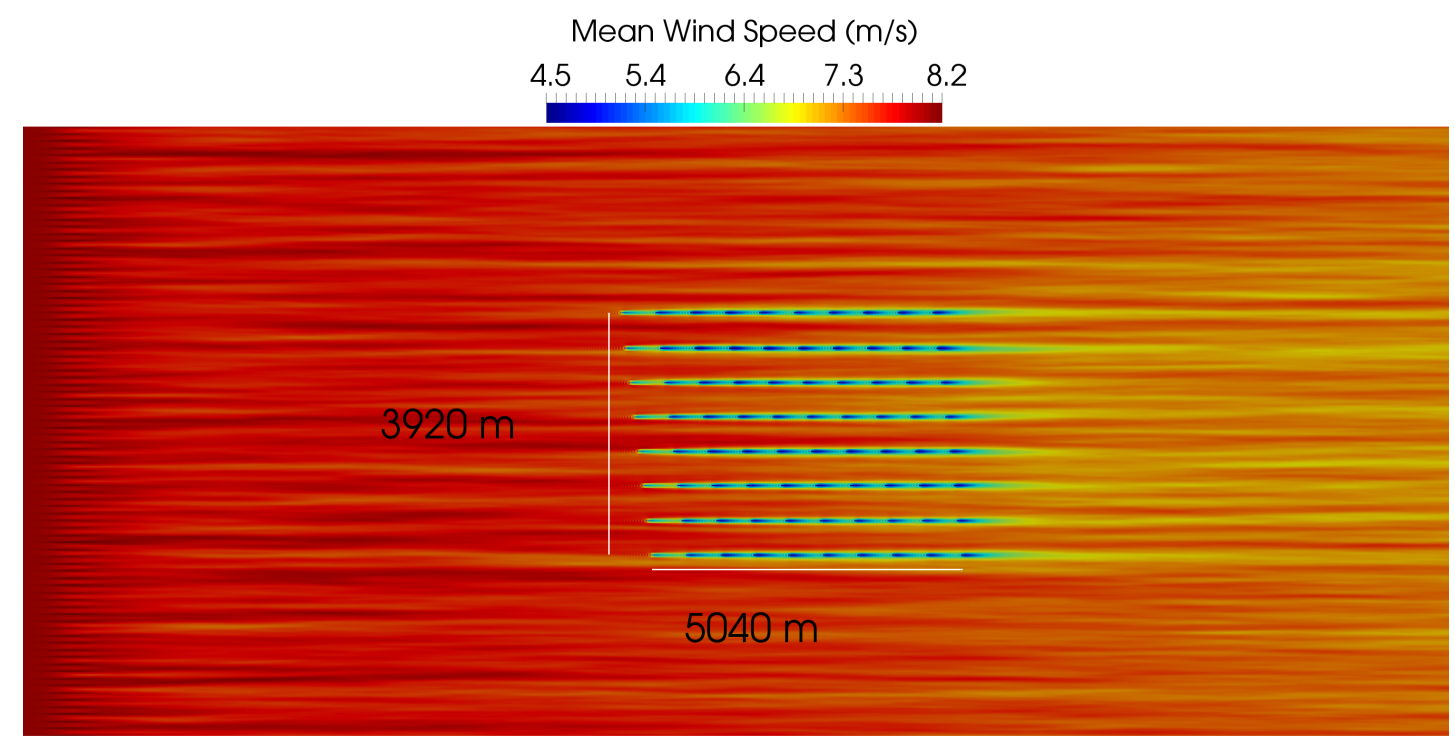

Figure 5.16: Visualization of the Horns Rev wind farm mean flow field showing the wind farm dimensions.

model performs better overall, but shows a large under-prediction of the normalized power at row 2 and a small under-prediction for the remainder of the rows. The current study performs well for the initial power drop, but does not capture the leveling off of power as well as $\mathrm{Wu}$ et al. [72]. It is possible that the results would be improved with an iterative rotational speed assignment with a relaxation coefficient as in $\mathrm{Wu}$ et al. [72].

\subsection{Application to a Wind Farm over Complex Terrain}

To show the versatility of the techniques presented in this thesis and their applicability to complex terrain, a section of land along the Snake River near Hagerman, Idaho is simulated. The domain is a sub-domain of a previous study on dynamic power line rating [47]. The goal of this simulation is to investigate the turbine power production difference possibly caused by the complex terrain and to be a proof of 


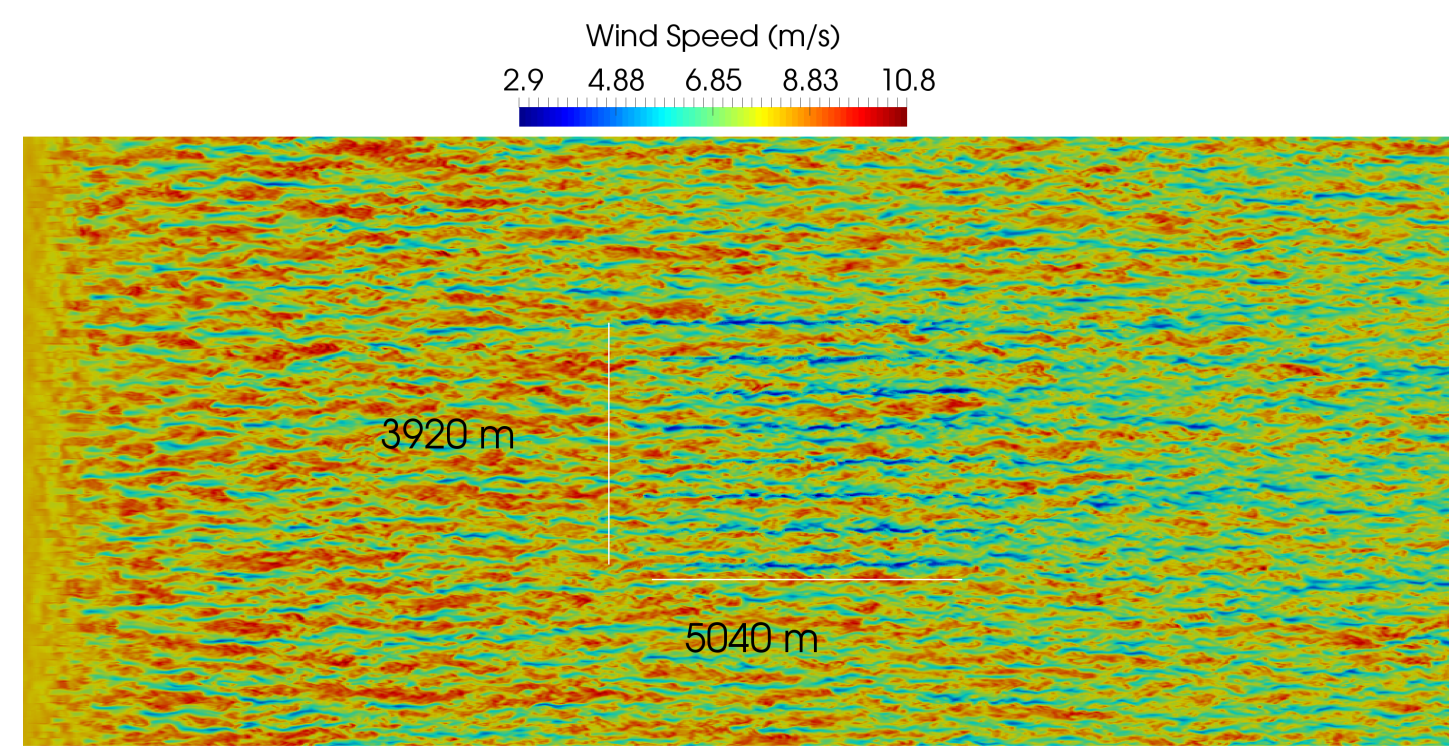

Figure 5.17: Visualization of the Horns Rev wind farm instantaneous flow field showing the wind farm dimensions.

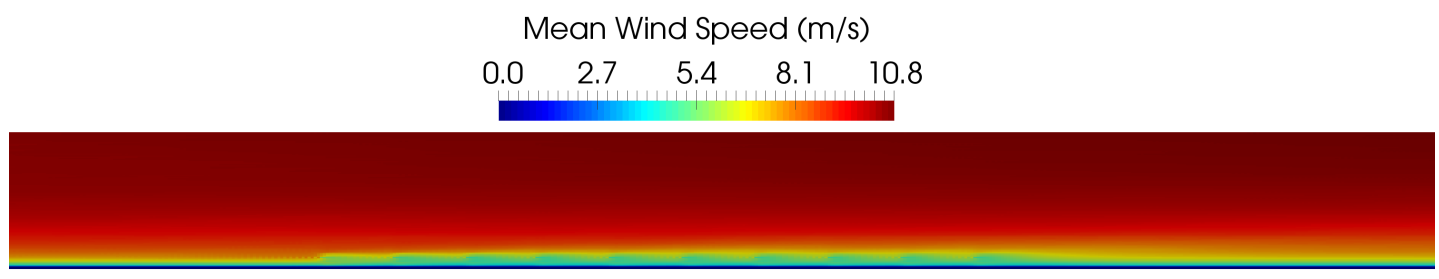

Figure 5.18: Visualization of the mean flow field around the first column of turbines in the Horns Rev wind farm.

concept of the methods discussed in this thesis. Eight turbines from an existing wind farm in the area are used, while nine additional turbines are added into the complex terrain in the canyon to investigate the impact of turbine siting on power production. The computational domain measures $22000 \mathrm{~m} \times 10000 \mathrm{~m} \times 1200 \mathrm{~m}$ with a grid size of $1025 \times 1025 \times 193$. The aerodynamic roughness length is set to $0.15 \mathrm{~m}$ and the RANS-LES blending height is $22.0 \mathrm{~m}$. The simulation is run for a total time of 3 hours with power data is collected over the last hour. Figure 5.22 shows a visualization of the flow in a portion of the domain. 


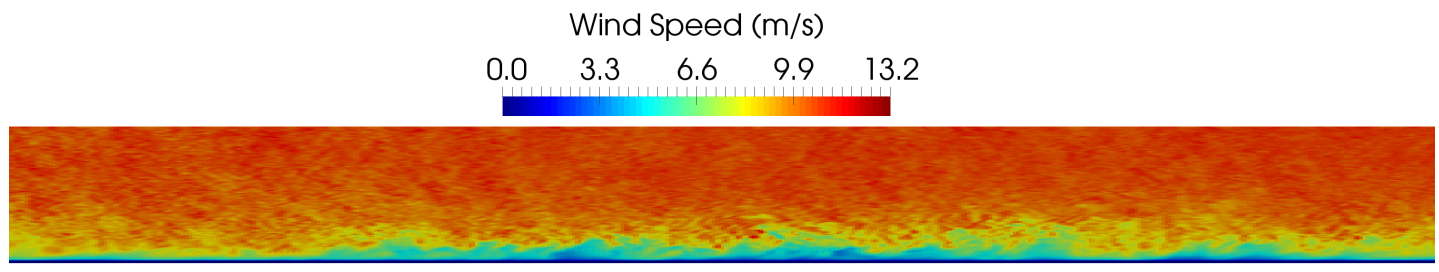

Figure 5.19: Visualization of the instantaneous flow field around the first column of turbines in the Horns Rev wind farm.

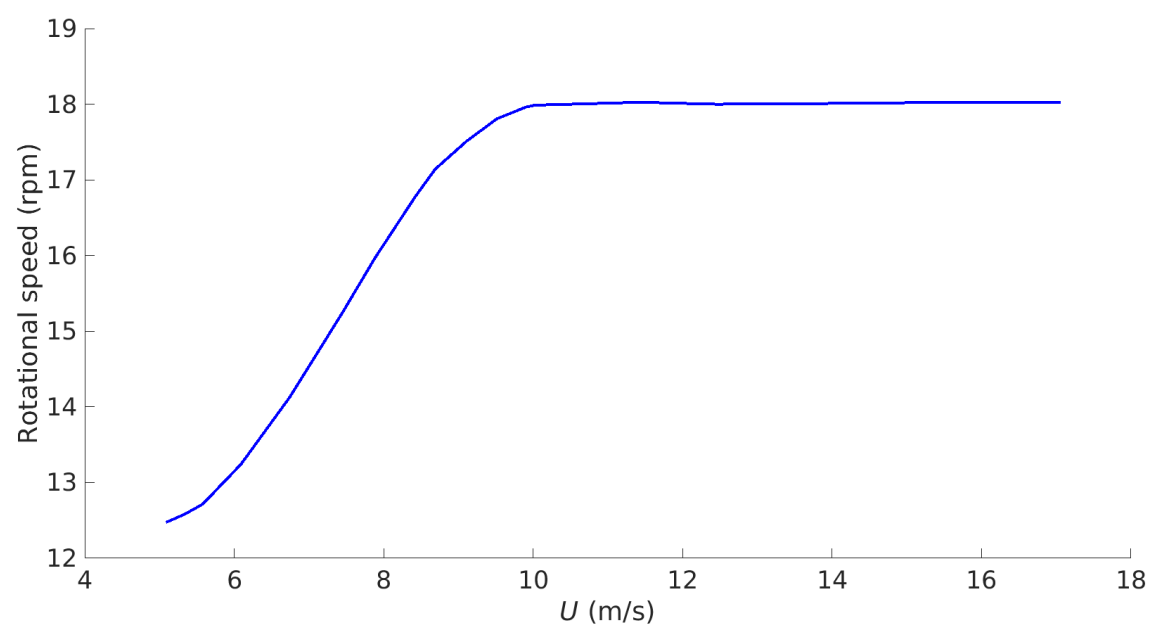

Figure 5.20: Reported angular speed as a function of wind speed for the Vestas V80 turbine. Data is from [72].

The turbines used are the Suzlon S88 2.1 MW turbines, which have a rotor diameter of $88 \mathrm{~m}$ and a hub height of $80 \mathrm{~m}$. The ADM-NR model is used due to the general unavailability of the blade characteristics. The thrust coefficient is 0.465 , which is derived from the manufacturer power curves [62], shown in Figure 4.1, for an upstream hub height wind speed of $10 \mathrm{~m} / \mathrm{s}$. The locations and model types for the existing wind turbines were found using the USGS windFarm tool [19]. The turbulent inflow method discussed in the previous section is used as well as periodic lateral boundary conditions and a stress free condition at the top of the domain.

The control volume method is applied to 3 upstream turbines not affected by 


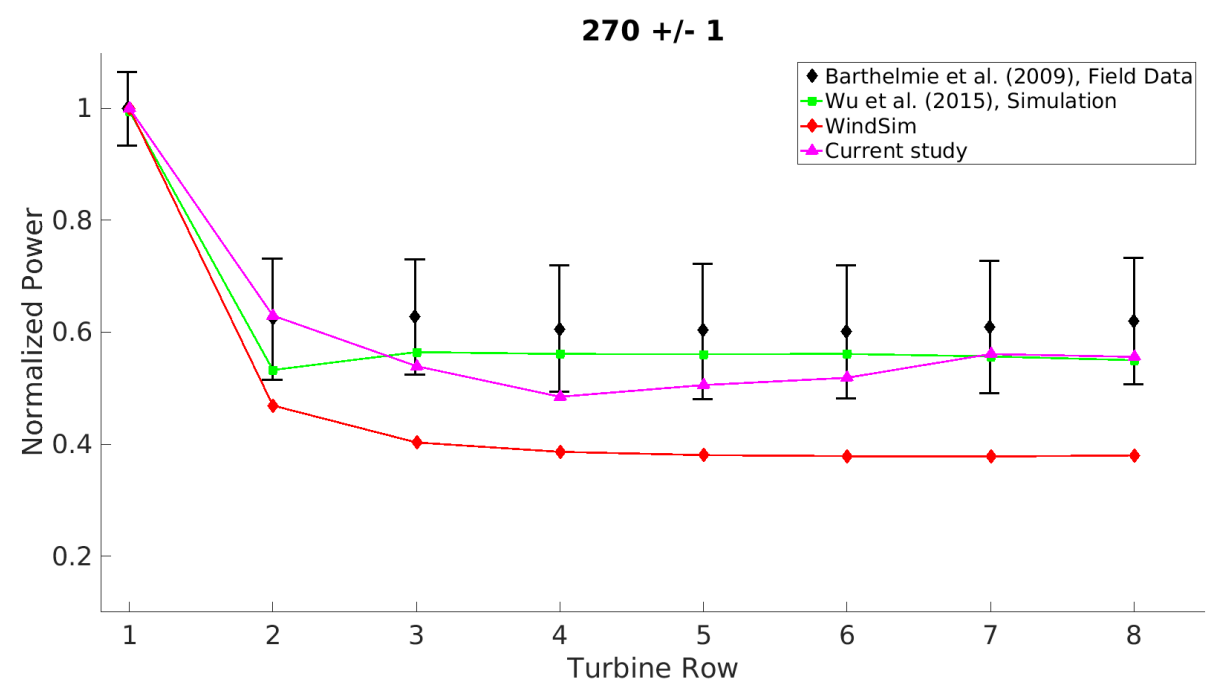

Figure 5.21: Average power at each turbine row normalized by power at row 1. Field data is reported in Barthelmie et al. [4] and the previous ADM-R simulation and WindSim [70] industry software results are reported in Wu et al. [72].

other turbine wakes both in the canyon and above the canyon. On average there was a $24 \%$ higher power extraction from the above canyon wind turbines, suggesting a significant influence on wind speed from the complex terrain. The results show a successful application of the methods discussed in this thesis. These methods have the potential to improve wind farm planning in complex and simple terrain and decrease the costs associated with wind resource assessment and wind farm planning. 


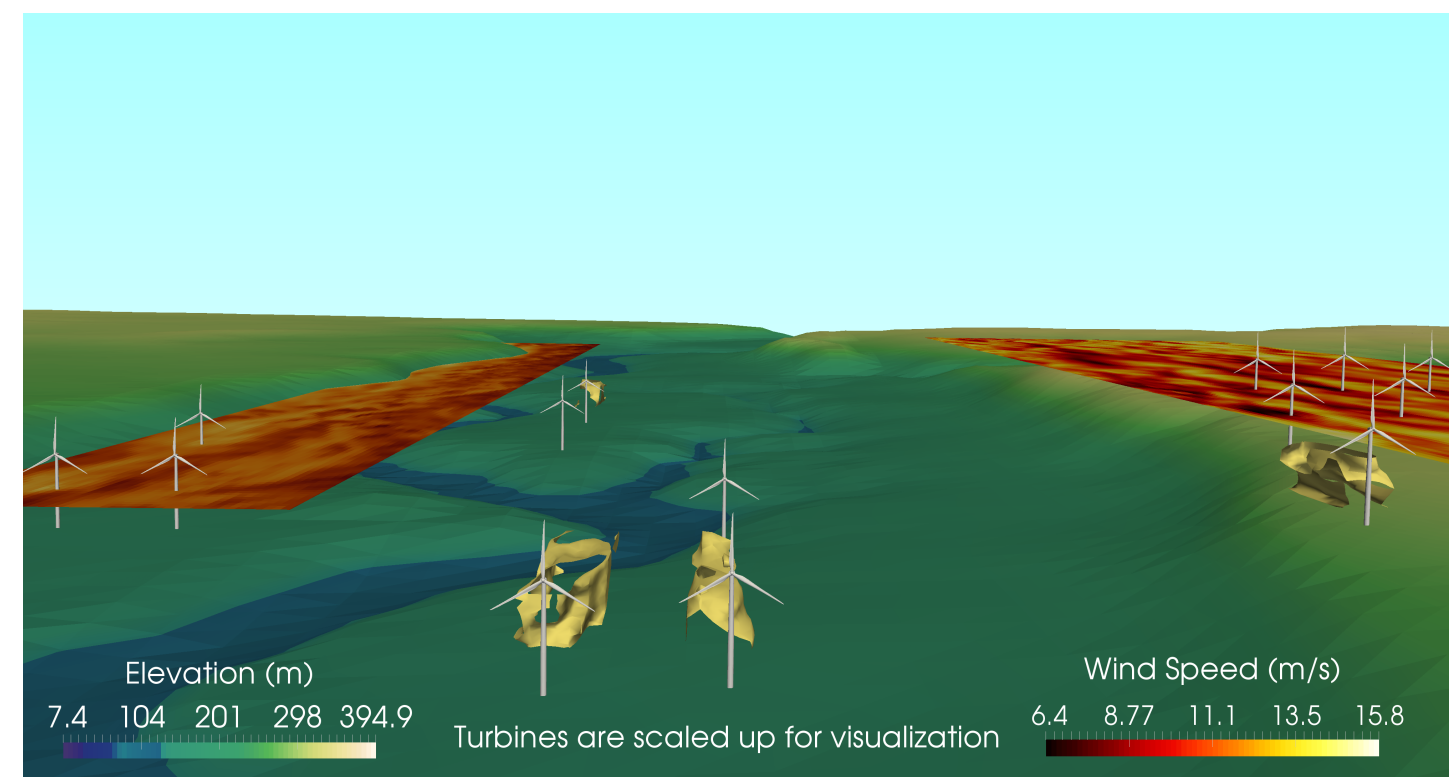

Figure 5.22: Visulization of the turbines, complex terrain, and flow field for a hypothetical wind farm in Hagerman, Idaho. 


\section{CHAPTER 6}

\section{SUMMARY}

\subsection{Wind Over Complex Terrain}

The IB method is well-suited for simulating turbulent wind flow over complex terrain. The fast, automated mesh generation and computational efficiency combined with the hybrid RANS-LES turbulence model is valuable for terrain flow simulations, especially large areas. We simulated wind flow over Askervein Hill and Bolund Hill to validate our methods. The simulations of Askervein Hill showed good agreement with the field study collection of velocity measurements for two different profiles over the hill. The simulations of Bolund Hill showed good agreement with the field study of Bolund hill in the vertical profiles as well as the detailed wind tunnel experiment. There was minor sensitivity to mesh resolution and turbulence model parameters. These benchmark cases showed that the IB method is well suited to produce accurate simulation data.

\subsection{Wake Models}

Wake models allow for accurate simulation of wind turbine wakes in large scale domains without the expensive task of resolving turbine blades. We applied and validated two wake models: the ADM-NR and the ADM-R. We explored both wind 
tunnel and full scale experiments as well as corresponding simulations to validate the models. The ADM-NR showed good agreement with the corresponding ADM-NR simulations, and the expected under-prediction of velocity deficit in the near wake region. The ADM-R showed good agreement with both the experimental data and corresponding simulations for the near and far wake regions. The ADM-R includes more accurate wind turbine physics and therefore produced better results than the ADM-NR.

\subsection{Control Volume Analysis for Power Estimation}

The variation in current power prediction methods and results suggest that a more fundamental approach could be used. We investigated the implementation of an energy budget to calculate the power extracted from the wind by single or multiple wind turbines. This control volume energy budget provides a robust method that is

derived from first-principle physics. The results from this method showed decent agreement with standard assessment methods in a flat terrain environment of an actual wind farm.

These methods were applied to assess the power from the large, operational offshore wind farm of Horns Rev, and a hypothetical wind farm located near Hagerman, Idaho along the Snake River. The simulation of the Horns Rev wind farm showed decent agreement with the field data and corresponding simulations and improvement over an industry software. The Hagerman wind farm is made up of 8 operational wind turbines as well as 9 theoretical wind turbines placed within the large canyon. The average power from the undisturbed, upstream turbines show a $24 \%$ higher power for turbines above the canyon, suggesting more favorable wind conditions in the less 
terrain affected areas of the domain.

The methods outlined in this thesis allow for simulation of winds and wind power assessment in complex terrain. The research represents the first application of a rotational wind turbine wake model to LES simulations of wind flow over complex terrain. Additionally, a first-principle based method for wind power assessment is developed and applied. These methods have the potential to aid in wind farm planning in order to reduce development costs and move closer to wind farm power forecasting with the utilization of high performance computing.

\subsection{Future Work}

To continue this research, future work would include a mesh refinement study to better understand the effects of mesh spacing on the $\mathrm{CV}$ method. Additional work would include validation of the proposed methods in complex terrain as more studies of wind power in complex terrain are conducted and published. To extend the capabilities of these methods, adaptive mesh refinement could be applied to the IB method, which would allow for pure LES simulations. 


\section{REFERENCES}

[1] EMD International A/S. windpro. https://www.emd.dk/windpro/, 2016.

[2] American Wind Energy Association. U.s. wind energy state facts. awea.org, 2016. Accessed: 2017.

[3] American Wind Energy Association. Wind energy facts at a glance. awea.org, 2017. Accessed: 2017-03-08.

[4] R.J. Barthelmie, K. Hansen, S.T. Frandsen, O. Rathmann, J.G. Schepers, W. Schlez, J. Phillips, K. Rados, A. Zervos, E.S. Politis, et al. Modelling and measuring flow and wind turbine wakes in large wind farms offshore. Wind Energy, 12(5):431-444, 2009.

[5] R.J. Barthelmie, S.C. Pryor, S.T. Frandsen, K.S. Hansen, J.G. Schepers, K. Rados, W. Schlez, A. Neubert, L.E. Jensen, and S. Neckelmann. Quantifying the impact of wind turbine wakes on power output at offshore wind farms. Journal of Atmospheric and Oceanic Technology, 27(8):1302-1317, 2010.

[6] A. Bechmann, N.N. Sørensen, J. Berg, J. Mann, and P.-E. Réthoré. The bolund experiment, part ii: blind comparison of microscale flow models. Boundary-Layer Meteorology, 141(2):245-271, 2011.

[7] J. Berg, J. Mann, A. Bechmann, M.S. Courtney, and H.E. Jørgensen. The bolund experiment, part i: Flow over a steep, three-dimensional hill. Boundary-layer meteorology, 141(2):219-243, 2011.

[8] M. Brower. Wind resource assessment: A practical guide to developing a wind project. https://www.awstruepower.com/assets/ Wind-Resource-Assessment-Practical-Guidance-for-Developing-a-Wind-Project-Bro pdf, 2012.

[9] M. Brower. Wind Resource Assessment: A Practical Guide to Developing a Wind Project. Wiley, 2012.

[10] T. Burton, D. Sharpe, N. Jenkins, and E. Bossanyi. Wind energy handbook. John Wiley \& Sons, 2001. 
[11] L.P. Chamorro, S.-J. Lee, D. Olsen, C. Milliren, J. Marr, R.E.A. Arndt, and F. Sotiropoulos. Turbulence effects on a full-scale $2.5 \mathrm{mw}$ horizontal-axis wind turbine under neutrally stratified conditions. Wind Energy, 18(2):339-349, 2015.

[12] L.P. Chamorro and F. Porté-Agel. Effects of thermal stability and incoming boundary-layer flow characteristics on wind-turbine wakes: A wind-tunnel study. Boundary-Layer Meteorology, 136(3):515-533, 2010.

[13] W.Y.Y. Cheng, Y. Liu, A.J. Bourgeois, Y. Wu, and S.E. Haupt. Short-term wind forecast of a data assimilation/weather forecasting system with wind turbine anemometer measurement assimilation. Renewable Energy, 107:340-351, 2017.

[14] A. Creech, W. Früh, and A.E. Maguire. Simulations of an offshore wind farm using large-eddy simulation and a torque-controlled actuator disc model. Surveys in Geophysics, 36(3):427-481, 2015.

[15] R. DeLeon, D. Jacobsen, and I. Senocak. Large-eddy simulations of turbulent incompressible flows on gpu clusters. Computing in Science $\mathscr{E}$ Engineering, 15(1):26-33, 2013.

[16] R. DeLeon, M. Sandusky, and I. Senocak. Simulations of turbulent winds over complex terrain using an immersed boundary method. Submitted to BoundaryLayer Meteorology.

[17] R. DeLeon and I. Senocak. Turbulent inflow generation for large-eddy simulation of high reynolds number incompressible flows through buoyancy perturbations. In AIAA AVIATION Forum, 2017.

[18] P. Devine-Wright. Beyond nimbyism: towards an integrated framework for understanding public perceptions of wind energy. Wind Energy, 8:125-139, 2005.

[19] Jay E Diffendorfer, Roger Compton, Louisa Kramer, Zach Ancona, and Donna Norton. Onshore industrial wind turbine locations for the united states. US Geological Survey Data Series, 817, 2014.

[20] D.L. Elliott, C.G. Holladay, W.R. Barchet, H.P. Foote, and W.F. Sandusky. Wind energy resource atlas of the united states. NASA STI/Recon Technical Report N, 87, 1987.

[21] DTU Wind Energy. Wasp. http://www.wasp.dk/, 2017.

[22] E.A. Fadlun, R. Verzicco, P. Orlandi, and J. Mohd-Yusof. Combined immersedboundary finite-difference methods for three-dimensional complex flow simulations. Journal of computational physics, 161(1):35-60, 2000. 
[23] A.M. Foley, P.G. Leahy, A. Marvuglia, and E.J. McKeogh. Current methods and advances in forecasting of wind power generation. Renewable Energy, 37(1):1-8, 2012.

[24] DNV GL. Windfarmer. https://www.dnvgl.com/services/windfarmer-3766, 2016.

[25] H. Glauert. Aerodynamic theory: A general review of progress, volume iv, chapter division 1, airplane propellers, 1963.

[26] G. Grötzbach. Direct numerical and large eddy simulation of turbulent channel flows. Encyclopedia of fluid mechanics, 6:1337-1391, 1987.

[27] P.L. Guth. Microdem. http://www.usna.edu/Users/oceano/pguth/website/ microdem/microdem.htm, 2013.

[28] D. Jacobsen and I. Senocak. A full-depth amalgamated parallel 3d geometric multigrid solver for gpu clusters. In 49th AIAA Aerospace Sciences Meeting including the New Horizons Forum and Aerospace Exposition, page 946, 2011.

[29] National Renewable Energy Laboratory. Wind turbine size increase 19802011. https://commons.wikimedia.org/wiki/File:Wind_turbine_size_ increase_1980-2011.png, 2012. Accessed: 2017-07-21.

[30] National Renewable Energy Laboratory. Wind maps. http://www.nrel.gov/ gis/wind.html, 2016. Accessed: 2017-03-08.

[31] J. Laursen, P. Enevoldsen, and booktitle=Proceedings of the European Wind Energy Conference, Milan, Italy volume $=610$ year $=2007$ Hjort, S. 3d cfd rotor computations of a multi-megawatt hawt rotor.

[32] ReSoft LTD. Windfarm from resoft. http://www.resoft.co.uk/English/ index.htm, 2016.

[33] H. Lu and F. Porté-Agel. Large-eddy simulation of a very large wind farm in a stable atmospheric boundary layer. Physics of Fluids, 23(6):065101, 2011.

[34] J.F. Manwell, J.G. McGowan, and A.L. Rogers. Wind energy explained: theory, design and application. John Wiley \& Sons, 2010.

[35] L. Martinez, S. Leonardi, M. Churchfield, and P. Moriarty. A comparison of actuator disk and actuator line wind turbine models and best practices for their use. In 50th AIAA Aerospace Sciences Meeting including the New Horizons Forum and Aerospace Exposition, page 900, 2012. 
[36] L.A. Martínez-Tossas, M.J. Churchfield, and S. Leonardi. Large eddy simulations of the flow past wind turbines: actuator line and disk modeling. Wind Energy, 18(6):1047-1060, 2015.

[37] B. McDonald. Surf2stl. http://www.mathworks.com/matlabcentral/ fileexchange/4512-surf2stl, 2004.

[38] C. Meneveau, T.S. Lund, and W.H. Cabot. A lagrangian dynamic subgrid-scale model of turbulence. Journal of Fluid Mechanics, 319:353-385, 1996.

[39] R. Mikkelsen. Actuator disc methods applied to wind turbines. $\mathrm{PhD}$ thesis, $\mathrm{PhD}$ thesis, Technical University of Denmark, 2003.

[40] A. Mittal, K. Sreenivas, L.K. Taylor, L. Hereth, C.B. Hilbert, and D.G. Hyams. Investigation of rotor models for wind turbine simulations. In 32nd AIAA Applied Aerodynamics Conference, page 2280, 2014.

[41] R. Mittal and G. Iaccarino. Immersed boundary methods. Annu. Rev. Fluid Mech., 37:239-261, 2005.

[42] J. Mohd-Yusof. Combined immersed-boundary $\backslash$ big-spline methods for simulations of flow in complex geometries. Annual Research Briefs, 317, 1997.

[43] B.R. Munson, A.P. Rothmayer, and T.H. Okiishi. Fundamentals of Fluid Mechanics, 7th Edition. Wiley, 2012.

[44] W. Munters, C. Meneveau, and J. Meyers. Shifted periodic boundary conditions for simulations of wall-bounded turbulent flows. Physics of Fluids, 28(2):025112, 2016.

[45] NikNaks. Atmospheric boundary layer. https://commons.wikimedia.org/ wiki/File:Atmospheric_boundary_layer.svg, 2012.

[46] C.S. Peskin. Flow patterns around heart valves: a numerical method. Journal of computational physics, 10(2):252-271, 1972.

[47] Tyler B Phillips, Inanc Senocak, Jake P Gentle, Kurt S Myers, and Phil Anderson. Investigation of a dynamic power line rating concept for improved wind energy integration over complex terrain. Tc, 273(100):100, 2014.

[48] E. S Politis, J. Prospathopoulos, D. Cabezon, K.S. Hansen, P.K. Chaviaropoulos, and R.J. Barthelmie. Modeling wake effects in large wind farms in complex terrain: the problem, the methods and the issues. Wind Energy, 15(1):161-182, 2012. 
[49] S.B. Pope. Turbulent flows, 771 pp, 2000.

[50] F. Porté-Agel, Y. Wu, H. Lu, and R.J. Conzemius. Large-eddy simulation of atmospheric boundary layer flow through wind turbines and wind farms. Journal of Wind Engineering and Industrial Aerodynamics, 99(4):154-168, 2011.

[51] L. Prandtl. Bericht über untersuchungen zur ausgebildeten turbulenz. Z. Angew. Math. Mech, 5(2):136-139, 1925.

[52] J.S. Rodrigo, P. Gancarski, R.C. Arroyo, P. Moriarty, M. Chuchfield, J. W Naughton, K.S. Hansen, E. Machefaux, T. Koblitz, E. Maguire, et al. Iea-task 31 wakebench: Towards a protocol for wind farm flow model evaluation. part 1: Flow-over-terrain models. In Journal of Physics: Conference Series, volume 524, page 012105. IOP Publishing, 2014.

[53] F. Roman, V. Armenio, and J. Fröhlich. A simple wall-layer model for large eddy simulation with immersed boundary method. Physics of Fluids, 21(10):101701, 2009.

[54] U. Schumann. Subgrid scale model for finite difference simulations of turbulent flows in plane channels and annuli. Journal of computational physics, 18(4):376404, 1975.

[55] I. Senocak, A.S. Ackerman, M.P. Kirkpatrick, D.E. Stevens, and N.N. Mansour. Study of near-surface models for large-eddy simulations of a neutrally stratified atmospheric boundary layer. Boundary-layer meteorology, 124(3):405-424, 2007.

[56] I Senocak, AS Ackerman, DE Stevens, and NN Mansour. Topography modeling in atmospheric flows using the immersed boundary method. 2004 Annual Research Briefs, Center for Turbulence Research, NASA Ames/Stanford Univ., Palo Alto, CA, pages 331-341, 2004.

[57] I. Senocak, M. Sandusky, R. DeLeon, D. Wade, K. Felzien, and M. Budnikova. An immersed boundary geometric preprocessor for arbitrarily complex terrain and geometry. Journal of Atmospheric and Oceanic Technology, 32(11):2075-2087, 2015 .

[58] J.N. Sørensen and W.Z. Shen. Numerical modeling of wind turbine wakes. J. Fluids Eng, 124(2):393-399, 2002.

[59] R.J.A.M. Stevens and C. Meneveau. Flow structure and turbulence in wind farms. Annual Review of Fluid Mechanics, 49:311-339, 2017.

[60] R.B. Stull. An Introduction to Boundary Layer Meteorology. Kluwer Academic Publishers, 1988. 
[61] S. Sunada, A. Sakaguchi, and K. Kawachi. Airfoil section characteristics at a low reynolds number. Journal of Fluids Engineering, 119:129, 1997.

[62] Suzlon. S88 - $2.1 \mathrm{mw}$ technical overview. http://www. suzlon. com, 2012.

[63] P.A. Taylor and H.W. Teunissen. The askervein hill project: Report on the sept./oct. 1983, main field experiment. Meteorological Services Research Branch, Atmospheric Environment Service, 1985.

[64] P.A. Taylor and H.W. Teunissen. The askervein hill project: Overview and background data. Boundary-Layer Meteorology, 39(1):15-39, 1987.

[65] J.C. Thibault and I. Senocak. Accelerating incompressible flow computations with a pthreads-cuda implementation on small-footprint multi-gpu platforms. The Journal of Supercomputing, 59(2):693-719, 2012.

[66] AWS Truepower. Openwind. http://software.awstruepower.com/ openwind/, 2017.

[67] C. Umphrey and I. Senocak. Turbulent inflow generation for the large-eddy simulation technique through globally neutral buoyancy perturbations. In 54th AIAA Aerospace Sciences Meeting, 2016.

[68] L.J. Vermeer, J. N. Sørensen, and A. Crespo. Wind turbine wake aerodynamics. Progress in aerospace sciences, 39(6):467-510, 2003.

[69] J.L. Walmsley and P.A. Taylor. Boundary-layer flow over topography: impacts of the askervein study. Boundary-Layer Meteorology, 78(3):291-320, 1996.

[70] WindSim. Windsim. https://www. windsim.com/, 2017.

[71] Y.-T. Wu and F. Porté-Agel. Large-eddy simulation of wind-turbine wakes: evaluation of turbine parametrisations. Boundary-layer meteorology, 138(3):345366, 2011.

[72] Y.-T. Wu and F. Porté-Agel. Modeling turbine wakes and power losses within a wind farm using les: An application to the horns rev offshore wind farm. Renewable Energy, 75:945-955, 2015.

[73] X. Yang, K.B. Howard, M. Guala, and F. Sotiropoulos. Effects of a threedimensional hill on the wake characteristics of a model wind turbine. Physics of Fluids, 27(2):025103, 2015.

[74] X. Yang, S. Kang, and F. Sotiropoulos. Computational study and modeling of turbine spacing effects in infinite aligned wind farms. Physics of Fluids, 24(11):115107, 2012. 
[75] X. Yang, F. Sotiropoulos, R.J. Conzemius, J.N. Wachtler, and M.B. Strong. Large-eddy simulation of turbulent flow past wind turbines/farms: the virtual wind simulator (vwis). Wind Energy, 18(12):2025-2045, 2015.

[76] T.S. Yeow, A. Cuerva-Tejero, and J. Perez-Alvarez. Reproducing the bolund experiment in wind tunnel. Wind Energy, 18(1):153-169, 2015.

[77] W. Zhang, C.D. Markfort, and F. Porté-Agel. Near-wake flow structure downwind of a wind turbine in a turbulent boundary layer. Experiments in fluids, 52(5):1219-1235, 2012.

[78] Hongkai Zhao. A fast sweeping method for eikonal equations. Mathematics of computation, 74(250):603-627, 2005. 